\title{
Asymmetric Transamination of $\alpha$-Keto Acids Catalyzed by Chiral Pyridoxamines
}

\author{
Xiaoyu Lan, Chuangan Tao, Xuliang Liu, Aina Zhang, Baoguo Zhao* \\ The Education Ministry Key Lab of Resource Chemistry and Shanghai Key Laboratory of Rare \\ Earth Functional Materials, Shanghai Normal University, Shanghai 200234, P. R. China \\ zhaobg2006@hotmail.com

\section{Supporting Information}

\section{Table of Contents}

General methods

S-2

Synthesis of compound 6

S-2

Synthesis of compound 7

Synthesis of compounds 8

Synthesis of compound 9

Synthesis of compounds $\mathbf{1 0}$

Synthesis of pyridoxamines 3d-f

Synthesis of pyridoxamines 3a-c and $\mathbf{3 g}$

Representative procedure for the asymmetric transamination

Characterization data

The X-ray structure of compound $(S, R)-\mathbf{8}$ 
General Methods. All commercially available reagents were used without further purification unless otherwise stated. Toluene and THF were freshly distilled from sodium-benzophenone under argon atmosphere. Dichloromethane was freshly distilled from $\mathrm{CaH}_{2}$. Methanol was freshly distilled after refluxing with magnesium turnings. Column chromatography was performed on silica gel (200-300 mesh). ${ }^{1} \mathrm{H}$ NMR spectra were recorded on a 400 or $600 \mathrm{MHz}$ NMR spectrometer and ${ }^{13} \mathrm{C}$ NMR spectra were recorded on a 100 or $150 \mathrm{MHz}$ NMR spectrometer. IR spectra were recorded on a FT-IR spectrometer. Melting points were uncorrected. $\alpha$-Keto acids including 2-oxopentanoic acid (11b), 2-oxooctanoic acid (11c), 2-oxo-3-phenylpropanoic acid (11j), 4-methyl-2-oxovaleric acid (11), and 2-oxopentanedioic acid (110) were purchased commercially. $\alpha$-Keto acids including 4-(naphthalen-1-yl)-2-oxobutanoic acid (11a), 2-oxotridec-12-enoic acid (11e), 8-(benzyloxy)-2-oxooctanoic acid (11f), 4-mesityl-2-oxobutanoic acid (11h), 4-(2,3-dihydrobenzofuran-5-yl)-2-oxobutanoic acid (11i), 4-ethyl-2-oxohexanoic acid (11m), and 2-oxo-4,4-diphenylbutanoic acid (11n) were prepared by addition of appropriate Grignard reagents to diethyloxalate followed by hydrolysis with acid or base. $^{1,2} \alpha$-Keto acids 2-oxodecanoic acid (11d) was prepared by addition of $n$-octylmagnesium bromide to mono-tert-butyloxalic acid- $N$-methoxy- $N$-methylamide followed by hydrolysis with acid. ${ }^{3.4} \alpha$-Keto acid 4-(biphenyl-2-yl)-2-oxobutanoic acid (11g) was prepared from 1,4-diacetylpiperazine-2,5-dione and 2-(biphenyl-2-yl)acetaldehyde by following literature procedure. ${ }^{5}$

\section{Procedure for Synthesis of Compound 6 (Scheme 2)}

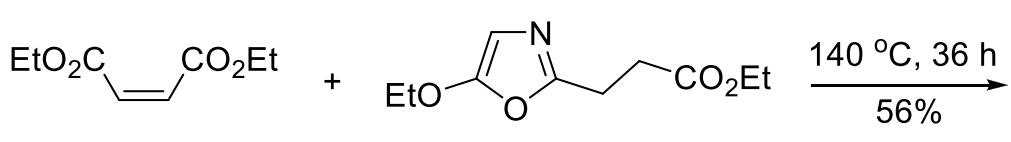
4
5<smiles>CCOCCc1ncc(O)c(C(=O)OCC)c1C(=O)OCC</smiles>

6 


\section{(1) Synthesis of compound 5}

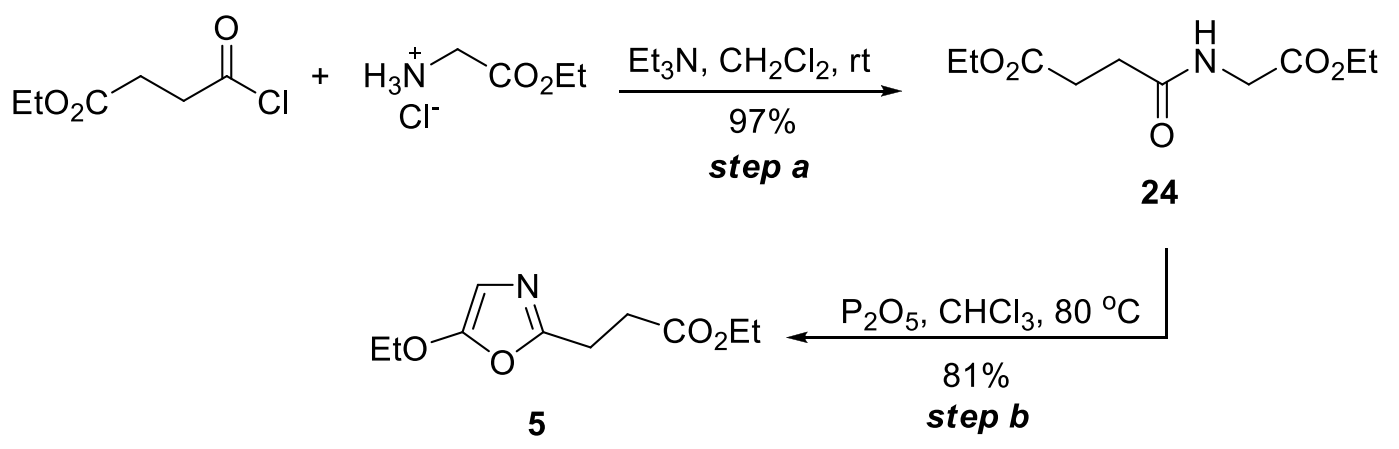

Step a: To a 2-L round-bottom flask vial equipped with a stirrer bar were added glycine ethyl ester hydrochloride (100.0 g, $716.4 \mathrm{mmol})$, dichloromethane (1000 mL), and dry $\mathrm{Et}_{3} \mathrm{~N}$ (145.2 $\left.\mathrm{g}, 1434.8 \mathrm{mmol}\right)$. After the mixture was cooled to $0{ }^{\circ} \mathrm{C}$ with ice bath, ethyl succinyl chloride (117.9 g, $716.3 \mathrm{mmol}$ ) was added dropwise over $30 \mathrm{~min}$. The reaction was allowed to warm up to room temperature and then stirred for $4 \mathrm{~h}$. The reaction mixture was washed with $\mathrm{H}_{2} \mathrm{O}(300 \mathrm{~mL})$ and saturated $\mathrm{NaHCO}_{3}$ aqueous solution $(300 \mathrm{~mL} \times 2)$, dried over anhydrous $\mathrm{Na}_{2} \mathrm{SO}_{4}$, filtered, and concentrated to give a white solid. The solid was dried over $\mathrm{P}_{2} \mathrm{O}_{5}$ in vaccum via oil pump to give the product $24(160.7 \mathrm{~g}, 97 \%)$.

24: White solid; m.p. $72-73{ }^{\circ} \mathrm{C}$; IR (KBr) 3321, 1748, 1734, 1655, 1552, 1212 $\mathrm{cm}^{-1} ;{ }^{1} \mathrm{H}$ NMR $\left(600 \mathrm{MHz}, \mathrm{CDCl}_{3}\right) \delta 6.29(\mathrm{~s}, 1 \mathrm{H}), 4.19(\mathrm{q}, J=7.2 \mathrm{~Hz}, 2 \mathrm{H}), 4.12(\mathrm{q}, J$ $=7.2 \mathrm{~Hz}, 2 \mathrm{H}), 4.00(\mathrm{~d}, J=4.8 \mathrm{~Hz}, 2 \mathrm{H}), 2.65(\mathrm{t}, J=6.6 \mathrm{~Hz}, 2 \mathrm{H}), 2.54(\mathrm{t}, J=6.6 \mathrm{~Hz}$, $2 \mathrm{H}), 1.26(\mathrm{t}, J=7.2 \mathrm{~Hz}, 3 \mathrm{H}), 1.23(\mathrm{t}, J=7.2 \mathrm{~Hz}, 3 \mathrm{H}) ;{ }^{13} \mathrm{C} \mathrm{NMR}\left(100 \mathrm{MHz}, \mathrm{CDCl}_{3}\right) \delta$ 172.7, 171.8, 169.8, 61.1, 60.4, 41.2, 30.3, 29.3, 13.93, 13.90; HRMS m/z Calcd. For $\mathrm{C}_{10} \mathrm{H}_{18} \mathrm{NO}_{5} \quad(\mathrm{M}+\mathrm{H})^{+}:$232.1185; Found: 232.1194 .

Step b: To a 2-L flame-dried, three-neck, round-bottom flask equipped with a stirrer bar and a reflux condenser was added trichloromethane $(1000 \mathrm{~mL})$. To the stirred trichloromethane were added phosphorus pentoxide (304 g, $2141.8 \mathrm{mmol}$ ) and a solution of compound $24(160.0 \mathrm{~g}, 693 \mathrm{mmol})$ in trichloromethane $(500 \mathrm{~mL})$. After the mixture was stirred at reflux for $8 \mathrm{~h}$, the solvent was immediately poured out. 
$\mathrm{NaHCO}_{3}(250 \mathrm{~g})$ was added and mixed together with the residue before the reaction mixture was cooled down. The purpose to introduce $\mathrm{NaHCO}_{3}$ immediately is to prevent the unreacted $\mathrm{P}_{2} \mathrm{O}_{5}$ to absorb moisture to form a whole sticky block. The mixed solid was slowly transferred to a container containing a stirred mixture of $\mathrm{NaHCO}_{3}(250 \mathrm{~g})$ and $\mathrm{H}_{2} \mathrm{O}(2000 \mathrm{~mL})$ at $0{ }^{\circ} \mathrm{C}$. After the reaction residue was completely dissolved, the mixture was extracted with ethyl acetate $(1000 \mathrm{~mL} \times 3)$. The combined organic layers were dried over anhydrous $\mathrm{Na}_{2} \mathrm{SO}_{4}$, filtered, concentrated via rotary evaporator under reduced pressure to give compound $\mathbf{5}$ as a colorless oil $(120.0 \mathrm{~g}, 81 \%)$.

5: Colorless oil; IR (KBr) 1734, 1689, 1620, 1593, $1099 \mathrm{~cm}^{-1} ;{ }^{1} \mathrm{H}$ NMR (400 MHz, $\left.\mathrm{CDCl}_{3}\right) \delta 5.88(\mathrm{~s}, 1 \mathrm{H}), 4.08(\mathrm{q}, J=7.2 \mathrm{~Hz}, 2 \mathrm{H}), 4.01(\mathrm{q}, J=7.2 \mathrm{~Hz}, 2 \mathrm{H}), 2.89(\mathrm{t}, J=$ $7.6 \mathrm{~Hz}, 2 \mathrm{H}), 2.67(\mathrm{t}, J=7.6 \mathrm{~Hz}, 2 \mathrm{H}), 1.33(\mathrm{t}, J=7.2 \mathrm{~Hz}, 3 \mathrm{H}), 1.18(\mathrm{t}, J=7.2 \mathrm{~Hz}, 3 \mathrm{H})$; HRMS $m / z$ Calcd. For $\mathrm{C}_{10} \mathrm{H}_{16} \mathrm{NO}_{4}(\mathrm{M}+\mathrm{H})^{+}:$214.1079; Found: 214.1078.

\section{(2) Synthesis of compound 6}

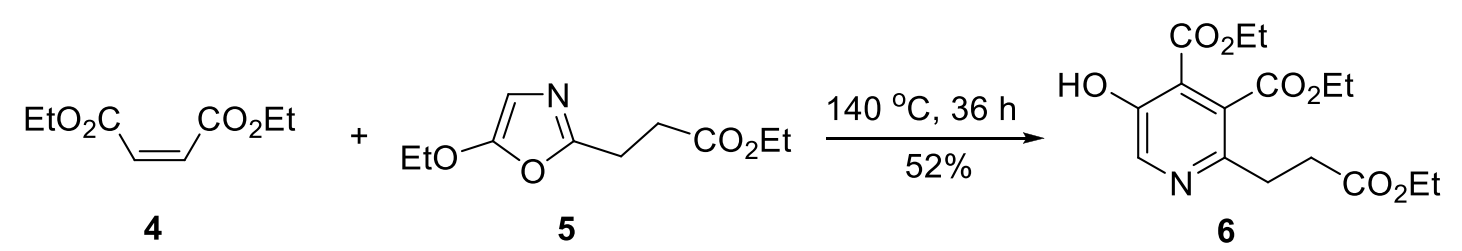

To a $500 \mathrm{~mL}$ round-bottom flask equipped with a magnetic stirrer bar were added compound 5 (120.0 g, $562.8 \mathrm{mmol}$ ) and diethyl maleate 4 (97.0, $563.4 \mathrm{mmol})$. The mixture was stirred at $140{ }^{\circ} \mathrm{C}$ for $36 \mathrm{~h}$. The reaction was completed as monitored by TLC. The mixture was purified by column chromatography on silica gel (petroleum ether / ethyl acetate $=5: 1)$ to give compound 6 as a light yellow solid (100.0 g, 52\%).

6: Light yellow solid; m.p. 37-38 ${ }^{\circ} \mathrm{C}$; IR (KBr) 3221, 1724, 1694, 1580, 1566, 1451, $1042 \mathrm{~cm}^{-1} ;{ }^{1} \mathrm{H}$ NMR (400 MHz, $\mathrm{CDCl}_{3}$ ) $\delta 10.38$ (s, 1H), 8.44 (s, 1H), 4.44-4.35 (m, 4H), $4.12(\mathrm{q}, J=7.2 \mathrm{~Hz}, 2 \mathrm{H}), 2.99$ (t, $J=7.6 \mathrm{~Hz}, 2 \mathrm{H}), 2.73(\mathrm{t}, J=7.6 \mathrm{~Hz}, 2 \mathrm{H})$, 1.43-1.32 (m, 6H), $1.22(\mathrm{t}, J=7.2 \mathrm{~Hz}, 3 \mathrm{H}) ;{ }^{13} \mathrm{C} \mathrm{NMR}\left(100 \mathrm{MHz}, \mathrm{CDCl}_{3}\right) \delta 172.7$, 
$167.7,167.2,153.4,146.9,142.2,126.9,115.2,63.0,61.9,60.3,33.1,29.6,14.1,14.0$, 13.7; HRMS $m / z$ Calcd. For $\mathrm{C}_{16} \mathrm{H}_{22} \mathrm{NO}_{7} \quad(\mathrm{M}+\mathrm{H})^{+}:$340.1396; Found: 340.1342 .

\section{Procedure for Synthesis of Compound 7 (Scheme 2)}<smiles>CCOCCc1ncc(O)c(C(=O)OCC)c1C(=O)OCC</smiles>

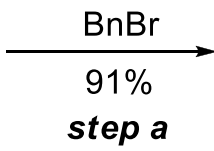<smiles>[3H]c1ncc(OCc2ccccc2)c(C(=O)OCC)c1C(=O)OCC</smiles>

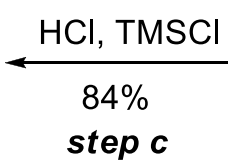<smiles>CCOC(=O)CCc1ncc(OCc2ccccc2)c(C(=O)OCC)c1C(=O)OCC</smiles>

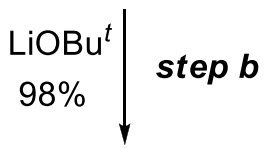<smiles>CCOC(=O)c1c(OCc2ccccc2)cnc2c1C(=O)C(C(=O)OCC)C2</smiles>

26

Step a: To a dry 1-L round-bottom flask equipped with a magnetic stirrer bar were added compound 6 (47.0 g, $138.5 \mathrm{mmol}), \mathrm{KCO}_{3}(23.0 \mathrm{~g}, 166.4 \mathrm{mmol})$, and dry acetonitrile $(500 \mathrm{~mL})$. After the mixture was stirred at $40{ }^{\circ} \mathrm{C}$ for $10 \mathrm{~min}$, benzyl bromide (23.8 g, $139.2 \mathrm{mmol})$ was added dropwise over $30 \mathrm{~min}$. After stirring at $40{ }^{\circ} \mathrm{C}$ for $5 \mathrm{~h}$, the mixture was filtered. The filtrate was concentrated via rotary evaporator under reduced pressure and purified by chromatography on silica gel (petroleum ether / ethyl acetate $=3: 1$ ) to give the product 25 as a yellow solid (54.0 g, $91 \%)$

25: Yellow solid; m.p. $45-47{ }^{\circ} \mathrm{C}$; IR (KBr) 1732, 1474, 1463, 1370, 1314, 1046 $\mathrm{cm}^{-1} ;{ }^{1} \mathrm{H}$ NMR (400 MHz, $\left.\mathrm{CDCl}_{3}\right) \delta 8.37(\mathrm{~s}, 1 \mathrm{H}), 7.41-7.30(\mathrm{~m}, 5 \mathrm{H}), 5.21(\mathrm{~s}, 2 \mathrm{H})$, 4.40-4.30 (m, 4H), $4.11(\mathrm{q}, J=7.2 \mathrm{~Hz}, 2 \mathrm{H}), 3.23(\mathrm{t}, J=7.6 \mathrm{~Hz}, 2 \mathrm{H}), 2.75$ (t, $J=7.6$ $\mathrm{Hz}, 2 \mathrm{H}), 1.36(\mathrm{t}, J=7.2 \mathrm{~Hz}, 3 \mathrm{H}), 1.31(\mathrm{t}, J=7.2 \mathrm{~Hz}, 3 \mathrm{H}), 1.21(\mathrm{t}, J=7.2 \mathrm{~Hz}, 3 \mathrm{H}) ;{ }^{13} \mathrm{C}$ NMR $\left(100 \mathrm{MHz}, \mathrm{CDCl}_{3}\right) \delta 172.9,165.9,165.0,151.3,149.3,137.4,135.6,131.3$, 128.6, 128.3, 127.1, 125.3, 71.6, 62.0, 61.9, 60.3, 33.0, 30.5, 14.2, 14.0, 13.9; HRMS $m / z$ Calcd. For $\mathrm{C}_{23} \mathrm{H}_{28} \mathrm{NO}_{7}(\mathrm{M}+\mathrm{H})^{+}:$430.1866; Found: 430.1881. 
Step b: To a dry 1-L round-bottom flask equipped with a magnetic stirrer bar were added compound $25(54.0 \mathrm{~g}, 125.7 \mathrm{mmol})$ and dry toluene $(500 \mathrm{~mL})$. After the mixture was cooled to $0{ }^{\circ} \mathrm{C}$ with ice bath, lithium tert-butoxide $(31.0 \mathrm{~g}, 387.2 \mathrm{mmol})$ was added. The reaction was allowed to warm up to room temperature and stirred for $5 \mathrm{~h}$ at the temperature. After the reaction was completed as monitored by TLC, the reaction mixture was poured into $\mathrm{HCl}$ solution $(1.0 \mathrm{M}, 390 \mathrm{~mL})$ at $0{ }^{\circ} \mathrm{C}$ and then extracted with ethyl acetate $(400 \mathrm{~mL} \times 2)$. The combined organic layers were dried over anhydrous $\mathrm{Na}_{2} \mathrm{SO}_{4}$, filtered, and concentrated via rotary evaporator under reduced pressure to give the product 26 as a white solid $(47.2 \mathrm{~g}, 98 \%)$.

26: White solid; m.p. $95-96{ }^{\circ} \mathrm{C}$; IR (KBr) 1734, 1637, 1566, 1369, 1309, 1150 $\mathrm{cm}^{-1}$; ${ }^{1} \mathrm{H}$ NMR $\left(400 \mathrm{MHz}, \mathrm{CDCl}_{3}\right){ }^{1} \mathrm{H}$ NMR $\left(400 \mathrm{MHz}, \mathrm{CDCl}_{3}\right.$ ) For enol form (85\%): $\delta 10.5(\mathrm{~s}, 1 \mathrm{H}), 8.34(\mathrm{~s}, 1 \mathrm{H}), 7.43-7.28(\mathrm{~m}, 5 \mathrm{H}), 5.23(\mathrm{~s}, 2 \mathrm{H}), 4.46(\mathrm{q}, J=7.2 \mathrm{~Hz}, 2 \mathrm{H})$, $4.33(\mathrm{q}, J=7.2 \mathrm{~Hz}, 2 \mathrm{H}), 3.55(\mathrm{~s}, 2 \mathrm{H}), 1.40-1.33$ (m, 6H); For ketone form (15\%): $\delta$ 8.59 (s, 1H), 7.43-7.28 (m, 5H), $5.26(\mathrm{~s}, 2 \mathrm{H}), 4.47$ (q, J = $7.2 \mathrm{~Hz}, 2 \mathrm{H}), 4.24$ (q, J = 7.2 $\mathrm{Hz}, 2 \mathrm{H}), 3.83(\mathrm{dd}, J=8.4,4.4 \mathrm{~Hz}, 1 \mathrm{H}), 3.62(\mathrm{dd}, J=17.6,4.4 \mathrm{~Hz}, 1 \mathrm{H}), 3.41(\mathrm{dd}, J=$ 17.6, 8.4 Hz, 1H), 1.37 (t, $J=7.2 \mathrm{~Hz}, 3 \mathrm{H}), 1.30$ (t, $J=7.2 \mathrm{~Hz}, 3 \mathrm{H})$; HRMS $m / z$ Calcd. For $\mathrm{C}_{21} \mathrm{H}_{22} \mathrm{NO}_{6}(\mathrm{M}+\mathrm{H})^{+}:$384.1447; Found: 384.1414 .

Step c: To a 1-L round-bottom flask equipped with a magnetic stirrer bar were added compound 26 (47.2 g, $123.1 \mathrm{mmol})$, ethanol (500 mL), concentrated hydrochloric acid $(53 \mathrm{~mL})$, and trimethylchlorosilane (114.2 g, $1051.2 \mathrm{mmol})$. After stirring at reflux for $17 \mathrm{~h}$, the mixture was submitted to filtration to give a red solid. The solid was dissolved in dichloromethane $(500 \mathrm{~mL})$. After the mixture was cooled to $0{ }^{\circ} \mathrm{C}$ with ice bath, $\mathrm{H}_{2} \mathrm{O}(500 \mathrm{~mL})$ and sodium bicarbonate $(32.0 \mathrm{~g})$ were added. The mixture was allowed to warm up to room temperature, stirred for $1 \mathrm{~h}$, and separated. The aqueous layer was extracted with dichloromethane $(300 \mathrm{~mL} \times 2)$. The combined organic layers were dried over anhydrous $\mathrm{Na}_{2} \mathrm{SO}_{4}$, filtered, and concentrated via rotary evaporator under reduced pressure to give the product 7 as a white solid $(32.0 \mathrm{~g}, 84 \%)$.

7: White solid; m.p. $154-156{ }^{\circ} \mathrm{C}$; IR (KBr) 1732, 1711, 1495, 1469, $1458 \mathrm{~cm}^{-1} ;{ }^{1} \mathrm{H}$ 
NMR (400 MHz, $\left.\mathrm{CDCl}_{3}\right) \delta 8.56(\mathrm{~s}, 1 \mathrm{H}), 7.41-7.29(\mathrm{~m}, 5 \mathrm{H}), 5.25(\mathrm{~s}, 2 \mathrm{H}), 4.69(\mathrm{q}, J=$ $7.2 \mathrm{~Hz}, 2 \mathrm{H}), 3.25-3.15(\mathrm{~m}, 2 \mathrm{H}), 2.82-2.75(\mathrm{~m}, 2 \mathrm{H}), 1.37(\mathrm{t}, J=7.2 \mathrm{~Hz}, 3 \mathrm{H}) ;{ }^{13} \mathrm{C} \mathrm{NMR}$ $\left(100 \mathrm{MHz}, \mathrm{CDCl}_{3}\right) \delta 202.7,166.4,163.8,149.7,143.2,135.4,128.6,128.3,127.2$, 127.0, 126.5, 72.1, 62.2, 36.6, 27.8, 14.0; HRMS m/z Calcd. For $\mathrm{C}_{18} \mathrm{H}_{18} \mathrm{NO}_{4}(\mathrm{M}+$ H) ${ }^{+}:$312.1236; Found: 312.1231.

\section{Procedure for Synthesis of Compounds 8 (Scheme 2)}<smiles>[3H]c1ncc(OCc2ccccc2)c(C(=O)OCC)c1C(=O)OCC</smiles>

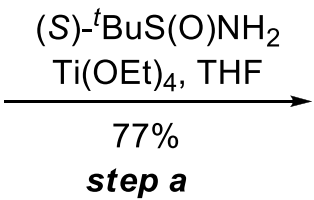
step a<smiles>CCOCc1c(OCC)cnc2c1/C(=N/S([15CH])=O)CC2</smiles>

27<smiles>CCCCCCCCS(=O)N[C@H]1CCc2ncc(OCc3ccccc3)c(COCC)c21</smiles>

$(S, S)-8(29 \%)$

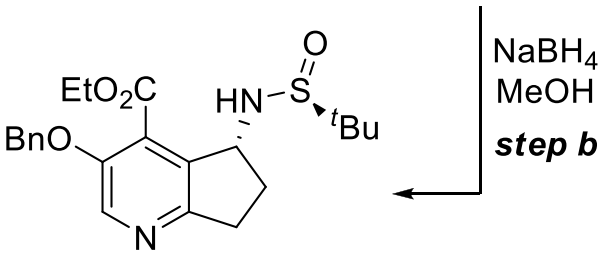

$(S, R)-8(52 \%)$

Step a: To a solution of compound $7\left(\begin{array}{lllll}10.0 & \mathrm{~g}, & 32.1 & \mathrm{mmol}\end{array}\right)$ and (S)-(-)-tert-butylsulfinamide $(38.9 \mathrm{~g}, 321.0 \mathrm{mmol})$ in anhydrous THF (500 $\mathrm{mL})$ was added $\mathrm{Ti}(\mathrm{OEt})_{4}(73.0 \mathrm{~g}, 320.0 \mathrm{mmol})$ under stirring. After stirring at reflux for $44 \mathrm{~h}$, the reaction mixture was cooled to room temperature and then submitted to rotary evaporation to remove the THF solvent. To the residue was added ethyl acetate ( 800 $\mathrm{mL})$. The mixture was slowly poured into a saturated $\mathrm{NaHCO}_{3}$ solution $(1000 \mathrm{~mL})$ under rapid stirring, stirred overnight, and filtered via a pad of cotton. The organic layer was collected and concentrated via rotary evaporator under reduced pressure. To the residue was added $\mathrm{H}_{2} \mathrm{O}(500 \mathrm{~mL})$. The mixture was stirred for $3 \mathrm{~h}$ and filtered to give a brown solid. The solid was further purified by column chromatography on silica gel (petroleum ether / ethyl acetate $=1: 1$ ) to give compound 27 as a yellow solid $(10.3 \mathrm{~g}, 77 \%)$. 
27: Yellow solid; m.p. $141-145{ }^{\circ} \mathrm{C} ;[\alpha]^{25}{ }_{\mathrm{D}}=-6.83\left(c 0.50, \mathrm{CH}_{3} \mathrm{OH}\right)$; IR $(\mathrm{KBr})$ 1739, 1614, 1565, 1489, $1308 \mathrm{~cm}^{-1} ;{ }^{1} \mathrm{H}$ NMR (400 MHz, $\mathrm{CDCl}_{3}$ ), $\delta 8.46$ (s, 1H), 7.39-7.29 (m, 5H), 5.22 (s, 2H), 4.37 (q, J = 7.2 Hz, 2H), 3.63-3.52 (m, H), 3.23-3.10 $(\mathrm{m}, 3 \mathrm{H}), 1.29$ (t, $J=7.2 \mathrm{~Hz}, 3 \mathrm{H}), 1.28(\mathrm{~s}, 9 \mathrm{H}) ;{ }^{13} \mathrm{C} \mathrm{NMR}\left(100 \mathrm{MHz}, \mathrm{CDCl}_{3}\right) \delta 179.5$, $164.5,162.9,150.3,141.4,135.6,128.8,128.7,128.5,127.5,127.3,72.2,62.0,58.2$, 31.5, 30.7, 22.5, 14.1; HRMS $m / z$ Calcd. For $\mathrm{C}_{22} \mathrm{H}_{27} \mathrm{~N}_{2} \mathrm{O}_{4} \mathrm{~S}(\mathrm{M}+\mathrm{H})^{+}$: 415.1692; Found: 415.1649.

Step b: To a stirred mixture of compound 27 (10.0 g, $24.1 \mathrm{mmol})$ and anhydrous $\mathrm{MeOH}(100 \mathrm{~mL})$ was slowly added $\mathrm{NaBH}_{4}(2.7 \mathrm{~g}, 71.4 \mathrm{mmol})$ at $-78{ }^{\circ} \mathrm{C}$. The mixture was stirred at $-78{ }^{\circ} \mathrm{C}$ for $30 \mathrm{~min}$, warmed up to room temperature, and stirred for additional $3 \mathrm{~h}$ at room temperature, quenched with saturated $\mathrm{NH}_{4} \mathrm{Cl}$ solution under ice bath until no gas came out, concentrated via rotary evaporator under reduced pressure to remove $\mathrm{CH}_{3} \mathrm{OH}$, and extracted with ethyl acetate $(300 \mathrm{~mL} \times 2)$. The combined organic layers were dried over $\mathrm{NaSO}_{4}$, filtered, concentrated, and purified by column chromatography on silica gel (petroleum ether / ethyl acetate $=1: 1$ ) to give compound $(S, S)-\mathbf{8}\left(\mathrm{R}_{\mathrm{f}}=0.3\right.$, petroleum ether / ethyl acetate $\left.=1: 1\right)(2.9 \mathrm{~g}, 29 \%)$ and $(S, R)-\mathbf{8}\left(\mathrm{R}_{\mathrm{f}}=\right.$ 0.2 , petroleum ether / ethyl acetate $=1: 1)(5.2 \mathrm{~g}, 52 \%)$. The total yield of the two diastereomers is $84 \%$ based on compound 27.

$(S, S)-8:$ White solid; m.p. $100-102{ }^{\circ} \mathrm{C} ;[\alpha]^{25}=116.2\left(c\right.$ 1.0, $\left.\mathrm{CH}_{3} \mathrm{OH}\right) ; \mathrm{IR}(\mathrm{KBr})$ $3185,1738,1500,1488 \mathrm{~cm}^{-1}$; ${ }^{1} \mathrm{H}$ NMR $\left(400 \mathrm{MHz}, \mathrm{CDCl}_{3}\right) \delta 8.29$ (s, 1H), 7.44-7.29 (m, 5H), $5.21(\mathrm{~d}, J=11.6 \mathrm{~Hz}, 1 \mathrm{H}), 5.17$ (d, $J=11.6 \mathrm{~Hz}, 1 \mathrm{H}), 4.98-4.92(\mathrm{~m}, 1 \mathrm{H}), 4.43$ (q, $J=7.2 \mathrm{~Hz}, 2 \mathrm{H}), 4.15(\mathrm{~s}, 1 \mathrm{H}), 3.19-3.07(\mathrm{~m}, 1 \mathrm{H}), 2.93-2.82(\mathrm{~m}, 1 \mathrm{H}),, 2.49-2.40(\mathrm{~m}$, $1 \mathrm{H}), 2.36-2.24(\mathrm{~m}, 1 \mathrm{H}), 1.35(\mathrm{t}, J=7.2 \mathrm{~Hz}, 3 \mathrm{H}), 1.19(\mathrm{~s}, 9 \mathrm{H})$.

$(S, R)-8$ : White solid; m.p. $136-138{ }^{\circ} \mathrm{C} ;[\alpha]^{25} \mathrm{D}=-168.1\left(c 0.5, \mathrm{CH}_{3} \mathrm{OH}\right) ; \mathrm{IR}(\mathrm{KBr})$ 3092, 1733, 1480, 1462, 1302, $1067 \mathrm{~cm}^{-1} ;{ }^{1} \mathrm{H}$ NMR (400 MHz, $\left.\mathrm{CDCl}_{3}\right) \delta 8.28(\mathrm{~s}, 1 \mathrm{H})$, 7.43-7.28 (m, 5H,), 5.19 (d, $J=12.0 \mathrm{~Hz}, 1 \mathrm{H}), 5.14$ (d, $J=12.0 \mathrm{~Hz}, 1 \mathrm{H}), 5.10-5.02(\mathrm{~m}$, $1 \mathrm{H}), 4.40-4.24(\mathrm{~m}, 2 \mathrm{H}), 3.59(\mathrm{~d}, J=9.6 \mathrm{~Hz}, 1 \mathrm{H}), 3.26-3.06(\mathrm{~m}, 1 \mathrm{H}), 2.94-2.83(\mathrm{~m}$, $1 \mathrm{H}), 2.74-2.62(\mathrm{~m}, 1 \mathrm{H}), 2.33-2.22(\mathrm{~m}, 1 \mathrm{H}), 1.29(\mathrm{t}, J=7.2 \mathrm{~Hz}, 3 \mathrm{H}), 1.18(\mathrm{~s}, 9 \mathrm{H}) ;{ }^{13} \mathrm{C}$ NMR $\left(100 \mathrm{MHz}, \mathrm{CDCl}_{3}\right) \delta 165.0,157.9,150.7,137.2,136.1,134.9,128.6,128.2$, 
127.8, 127.3, 72.0, 61.8, 59.7, 56.2, 33.6, 31.3, 22.6, 14.1; HRMS m/z Calcd. For $\mathrm{C}_{22} \mathrm{H}_{29} \mathrm{~N}_{2} \mathrm{O}_{4} \mathrm{~S}(\mathrm{M}+\mathrm{H})^{+}:$417.1848; Found: 417.1821 .

\section{Synthesis of Compound 9 (Scheme 2)}<smiles>CCOC(=O)c1c(OCC)cnc2c1CCC2</smiles>

$(S, R)-8$

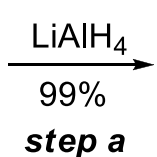

step a<smiles>CC(C)(C)S(=O)NC1CCc2ncc(OCc3ccccc3)c(CO)c21</smiles>

28<smiles>NC1CCc2nc(O)c(CO)c(Cl)c21</smiles>

9

Step a: To a stirred solution of compound $(S, R)-8(5.2 \mathrm{~g}, 12.5 \mathrm{mmol})$ in anhydrous THF $(100 \mathrm{~mL})$ was slowly added $\mathrm{LiAlH}_{4}(1.0 \mathrm{~g}, 26.4 \mathrm{mmol})$ at $-78{ }^{\circ} \mathrm{C}$. The mixture was stirred at $-78{ }^{\circ} \mathrm{C}$ for 30 min, warmed up to room temperature, and then stirred at room temperature for additional $1 \mathrm{~h}$. THF $(100 \mathrm{~mL})$ was added. The mixture was cooled down to $0{ }^{\circ} \mathrm{C}$ and then quenched by successive addition of water $(1.0 \mathrm{~mL})$ and aqueous sodium hydroxide solution $(1.0 \mathrm{~mL}, 10 \% \mathrm{~m} / \mathrm{m})$. After stirring at room temperature for $1 \mathrm{~h}$, the reaction mixture was filtered via a pad of celite. The filtrate was concentrated via rotary evaporation. To the residue were added ethyl acetate (20 $\mathrm{mL})$ and petroleum ether $(20 \mathrm{~mL})$. The resulting mixture was stirred at room temperature for $30 \mathrm{~min}$ and then submitted to filtration to give the product $\mathbf{2 8}$ as a white solid $(4.7 \mathrm{~g}, 99 \%)$.

28: White solid; m.p. $172-174{ }^{\circ} \mathrm{C} ;[\alpha]^{25}{ }_{\mathrm{D}}=-71.4\left(c\right.$ 1.0, $\left.\mathrm{CH}_{3} \mathrm{OH}\right)$; IR $(\mathrm{KBr}) 3378$, 3066, 3036, 1483, $1373 \mathrm{~cm}^{-1} ;{ }^{1} \mathrm{H}$ NMR (400 MHz, $\mathrm{CDCl}_{3}$ ) $\delta 8.18$ (s, 1H), 7.44-7.30 $(\mathrm{m}, 5 \mathrm{H}), 5.17(\mathrm{~d}, J=11.6 \mathrm{~Hz}, 1 \mathrm{H}), 5.14(\mathrm{~d}, J=11.6 \mathrm{~Hz}, 1 \mathrm{H}), 5.15-5.08(\mathrm{~m}, 1 \mathrm{H}), 4.89$ $(\mathrm{dd}, J=13.6,6.8 \mathrm{~Hz}, 1 \mathrm{H}), 4.76(\mathrm{dd}, J=13.6,5.6 \mathrm{~Hz}, 1 \mathrm{H}), 3.93(\mathrm{~d}, J=8.0 \mathrm{~Hz}, 1 \mathrm{H})$, $3.84(\mathrm{dd}, J=6.8,5.6 \mathrm{~Hz}, 1 \mathrm{H}), 3.17-3.05(\mathrm{~m}, 1 \mathrm{H}), 2.90-2.78(\mathrm{~m}, 1 \mathrm{H}), 2.59-2.45(\mathrm{~m}$, 1H), 2.30-2.20 (m, 1H), $1.20(\mathrm{~s}, 9 \mathrm{H}) ;{ }^{13} \mathrm{C} \mathrm{NMR}\left(100 \mathrm{MHz}, \mathrm{CDCl}_{3}\right) \delta 158.8,153.5$, 138.0, 137.8, 137.7, 135.6, 129.6, 129.1, 128.6, 72.4, 60.2, 57.2, 56.4, 33.8, 31.7, 23.1; HRMS $m / z$ Calcd. For $\mathrm{C}_{20} \mathrm{H}_{27} \mathrm{~N}_{2} \mathrm{O}_{3} \mathrm{~S}(\mathrm{M}+\mathrm{H})^{+}: 375.1742$; Found: 375.1732 . 
Step b: To a 1-L round-bottom flask equipped with a magnetic stirrer bar were added compound $28(2.0 \mathrm{~g}, 5.3 \mathrm{mmol})$ and $\mathrm{HCl}$ aqueous solution (400 mL, 6.0 M). The reaction was refluxed at $150{ }^{\circ} \mathrm{C}$ for $4 \mathrm{~h}$ and then concentrated via rotary evaporation under reduced pressure. To the residue was added dichloromethane (50 $\mathrm{mL}$ ). After stirring at room temperature for $10 \mathrm{~min}$, the mixture was filtered. The solid collected and dried in vacuum via oil pump to give the compound 9 as a white solid $(1.3 \mathrm{~g}, 97 \%)$.

9: White solid; m.p. $185-187{ }^{\circ} \mathrm{C}$; $[\alpha]^{25}{ }_{\mathrm{D}}=-22.5\left(c 0.50, \mathrm{CH}_{3} \mathrm{OH}\right)$; IR $(\mathrm{KBr}) 3373$, 3285, 3054, 1441, 1472, 1401, $1387 \mathrm{~cm}^{-1} ;{ }^{1} \mathrm{H}$ NMR (600 MHz, DMSO-d $)_{6} \delta 11.92$ (s, $1 \mathrm{H}), 8.55(\mathrm{~s}, 3 \mathrm{H}), 8.32(\mathrm{~s}, 1 \mathrm{H}), 5.22-5.16(\mathrm{~m}, 1 \mathrm{H}), 4.87(\mathrm{~d}, J=17.4 \mathrm{~Hz}, 1 \mathrm{H}), 4.82(\mathrm{~d}$, $J=17.4 \mathrm{~Hz}, 1 \mathrm{H}), 3.48-3.38(\mathrm{~m}, 1 \mathrm{H}), 3.11-3.02(\mathrm{~m}, 1 \mathrm{H}), 2.56-2.47$ (m, 1H), 2.34-2.26 $(\mathrm{m}, 1 \mathrm{H}) ;{ }^{13} \mathrm{C}$ NMR $\left(100 \mathrm{MHz}, \mathrm{DMSO}-d_{6}\right) \delta 151.9,151.0,145.9,136.2,126.3,57.7$, 52.2, 28.5, 27.8; HRMS m/z Calcd. For $\mathrm{C}_{9} \mathrm{H}_{13} \mathrm{~N}_{2} \mathrm{O}_{2}(\mathrm{M}+\mathrm{H})^{+}$: 181.0977; Found: 181.0950 .

Procedures for the Synthesis of Compounds 10a-g using 10e $\left(\mathrm{R}=\mathrm{CH}_{2} \mathrm{NBocCH}_{3}\right)$ as the representative example (Scheme 2)<smiles>N[C@H]1CCc2c1ncc(O)c2Cl</smiles>

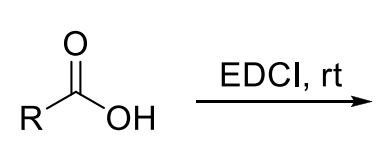

29 29a: $\mathrm{R}=n-\mathrm{Pr}$

29b: $\mathrm{R}=\mathrm{CH}_{2} \mathrm{OMe}$

29c: $\mathrm{R}=\mathrm{CH}_{2} \mathrm{NHAC}$

29d: $\mathrm{R}=\mathrm{CH}_{2} \mathrm{NHBOC}$

29e: $\mathrm{R}=\mathrm{CH}_{2} \mathrm{NMeBoc}$

29f: $\mathrm{R}=\mathrm{CH}_{2} \mathrm{NEtBoC}$

29g: $\mathrm{R}=\mathrm{CH}_{2} \mathrm{NMe}_{2}$<smiles>[R]C(=O)N[C@H]1CCc2ncc(O)c(CO)c21</smiles>

10a: $\mathrm{R}=n-\mathrm{Pr}$

10b: $\mathrm{R}=\mathrm{CH}_{2} \mathrm{OMe}$

10c: $\mathrm{R}=\mathrm{CH}_{2} \mathrm{NHAc}$

10d: $\mathrm{R}=\mathrm{CH}_{2} \mathrm{NHBoc}$

10e: $\mathrm{R}=\mathrm{CH}_{2} \mathrm{NMeBoC}$

10f: $\mathrm{R}=\mathrm{CH}_{2} \mathrm{NEtBoc}$

10g: $\mathrm{R}=\mathrm{CH}_{2} \mathrm{NMe}_{2}$

To a $100 \mathrm{~mL}$ round-bottom flask equipped with a magnetic stirrer bar were added compound 9 (3.86 g, $15.3 \mathrm{mmol})$ and carboxylic acid $29 \mathrm{e}(2.27 \mathrm{~g}, 12.0 \mathrm{mmol}), \mathrm{Et}_{3} \mathrm{~N}$ 
(4.86 g, $48.0 \mathrm{mmol}$ ), and dry dimethylformamide (35 mL). After the mixture was stirred at room temperature for $30 \mathrm{~min}$, ethyl cyanoglyoxalate-2-oxime (1.7 g, 12.0 mmol) was added. The resulting mixture was stirred at room temperature for another $30 \mathrm{~min}$, then $N$-(3-dimethylaminopropyl)- $N$ '-ethylcarbodiimide hydrochloride (EDCI) (3.4 $\mathrm{g}, 17.7 \mathrm{mmol})$ was added in one portion. The reaction mixture was stirred at room temperature for $4 \mathrm{~h}$ and then concentrated via rotary evaporation under reduced pressure. The residue was submitted to flash chromatography on silica gel (dichloromethane $/$ methanol $=10: 1)$ to give compound 10e as a white solid $(2.2 \mathrm{~g}$, $52 \%)$

10e: White solid; m.p. $198-202^{\circ} \mathrm{C} ;[\alpha]^{25}{ }_{\mathrm{D}}=23.6\left(c\right.$ 0.50, $\left.\mathrm{CH}_{3} \mathrm{OH}\right)$; IR (KBr) 3273, 3082, 1701, 1655, 1554, $1394 \mathrm{~cm}^{-1} ;{ }^{1} \mathrm{H}$ NMR (600 MHz, CD 3 OD) $\delta 7.94$ (s, 1H), 5.61-5.57 (m, 1H), $4.74(\mathrm{~d}, J=13.2 \mathrm{~Hz}, 1 \mathrm{H}), 4.69(\mathrm{~d}, J=13.2 \mathrm{~Hz}, 1 \mathrm{H}), 3.87(\mathrm{~d}, J=$ $15.6 \mathrm{~Hz}, 1 \mathrm{H}), 3.83(\mathrm{~d}, J=15.6 \mathrm{~Hz}, 1 \mathrm{H}), 3.11-3.03(\mathrm{~m}, 1 \mathrm{H}), 2.93(\mathrm{~s}$, Me for the cis or trans amide isomer), 2.92 (s, Me for the trans or cis amide isomer), 2.85-2.76 (m, 1H), 2.58-2.40 $(\mathrm{m}, 1 \mathrm{H}), 2.05-1.97(\mathrm{~m}, 1 \mathrm{H}), 1.45(\mathrm{~s}$, tert-Bu for the cis or trans amide isomer), 1.44 (s, tert-Bu for the trans or cis amide isomer); ${ }^{13} \mathrm{C} \mathrm{NMR} \mathrm{(150} \mathrm{MHz,}$ $\mathrm{CD}_{3} \mathrm{OD}$ ) for the cis and trans amide isomers: $\delta$ 170.9, 170.7, 158.1, 157.6, 156.6, $153.1,137.9,136.5,134.4,134.3,81.5,57.53,57.48,53.1,52.69,52.65,36.5,36.3$, 33.1, 32.9, 31.5, 28.6; HRMS $m / z$ Calcd. For $\mathrm{C}_{17} \mathrm{H}_{26} \mathrm{~N}_{3} \mathrm{O}_{5}(\mathrm{M}+\mathrm{H})^{+}:$352.1872; Found: 352.1877. 
<smiles>[R]C(=O)N[C@H]1CCc2ncc(O)c(CO)c21</smiles>

10d-f<smiles>[R]C(=O)N[C@H]1CC[C@H](C)c2c1ncc(O)c2O</smiles>

3d-f

3d: $\mathrm{R}=\mathrm{CH}_{2} \mathrm{NH}_{2}$ 3e: $\mathrm{R}=\mathrm{CH}_{2} \mathrm{NHMe}$ 3f: $\mathrm{R}=\mathrm{CH}_{2} \mathrm{NHEt}$

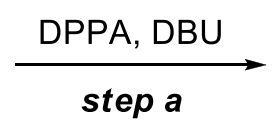

$\frac{\mathrm{TMSCl}-\mathrm{MeOH}}{\text { step c }}$<smiles>[R]C(=O)N[C@H]1CCc2ncc(O)c(CN)c21</smiles>

30d-f

$\mathrm{Pd} / \mathrm{C}, \mathrm{H}_{2}$ step $b$<smiles>[R]C(=O)N[C@H]1CCc2ncc([AsH2])c(CN)c21</smiles>

10d, 30d, and 31d: $\mathrm{R}=\mathrm{CH}_{2} \mathrm{NHBoc}$ 10e, 30e, and 31e: $\mathrm{R}=\mathrm{CH}_{2} \mathrm{NMeBoC}$ 10f, 30f, and 31f: $\mathrm{R}=\mathrm{CH}_{2} \mathrm{NEtBoc}$

Step a: To the mixture of compound 10e $(2.2 \mathrm{~g}, 6.3 \mathrm{mmol})$ and dry THF (30 mL) were added diphenylphosphoryl azide (DPPA) (2.55 g, $9.3 \mathrm{mmol})$ and 1,8-diazabicyclo[5.4.0]-7-undecene (DBU) $(2.44 \mathrm{~g}, 16.1 \mathrm{mmol})$ at $0{ }^{\circ} \mathrm{C}$. The mixture was stirred at room temperature for $8 \mathrm{~h}$ and then concentrated via rotary evaporation under reduced pressure. The residue was submitted to flash chromatography on silica gel (petroleum ether / acetone $=1: 1)$ to give compound 30e as a white solid $(1.2 \mathrm{~g}$, $51 \%)$.

Step $\boldsymbol{b}$ : The obtained azide 30e (1.2 g) was dissolved in dry THF (30 mL). To the solution was added $\mathrm{Pd} / \mathrm{C}\left(0.4 \mathrm{~g}, 10 \% \mathrm{Pd}\right.$ on carbon, wetted with $\left.55 \% \mathrm{H}_{2} \mathrm{O}\right)$. The mixture was stirred under 1 atm of $\mathrm{H}_{2}$ atmosphere at room temperature for $4 \mathrm{~h}$ and then filtered. The filtrate was dried over $\mathrm{Na}_{2} \mathrm{SO}_{4}$, filtered, and concentrated via rotary evaporation under reduced pressure. The residue was submitted to flash chromatography on silica gel (dichloromethane / methanol $=5: 1$ ) to give compound 31e as a white solid $(1.0 \mathrm{~g}, 90 \%)$. 
31e: White solid; m. p. $165-169{ }^{\circ} \mathrm{C} ;[\alpha]^{25}{ }_{\mathrm{D}}=29.7\left(c 0.50, \mathrm{CH}_{3} \mathrm{OH}\right)$; IR $(\mathrm{KBr})$ 3268, 1702, 1651, 1544, 1460, $1393 \mathrm{~cm}^{-1} ;{ }^{1} \mathrm{H}$ NMR (600 MHz, CD $\left.{ }_{3} \mathrm{OD}\right) \delta 7.87$ (s, $1 \mathrm{H}), 5.54-5.48(\mathrm{~m}, 1 \mathrm{H}), 4.07-3.77(\mathrm{~m}, 4 \mathrm{H}), 3.12-3.02(\mathrm{~m}, 1 \mathrm{H}), 2.98-2.88(\mathrm{~m}, 3 \mathrm{H}$ for the Me of trans/cis amide isomers), 2.84-2.75 (m, 1H), 2.57-2.48 (m, 1H), 2.04-1.92 $(\mathrm{m}, 1 \mathrm{H}), 1.50-1.37\left(\mathrm{~m}, 9 \mathrm{H}\right.$ for the tert-Bu groups of the trans/cis amide isomers); ${ }^{13} \mathrm{C}$ NMR (150 MHz, $\left.\mathrm{CD}_{3} \mathrm{OD}\right) \delta 170.7,170.6,158.0,157.6,157.0,156.7,154.4,154.2$, 139.2, 136.2, 136.0, 132.0, 131.4, 81.5, 53.0, 52.7, 52.5, 52.4, 39.4, 39.2, 36.6, 36.3, 33.1, 32.8, 31.5, 28.7; HRMS $m / z$ Calcd. For $\mathrm{C}_{17} \mathrm{H}_{27} \mathrm{~N}_{4} \mathrm{O}_{4}(\mathrm{M}+\mathrm{H})^{+}:$351.2032; Found: 351.2031 .

Step c: To a stirred solution of TMSCl $(6.21 \mathrm{~g}, 57.1 \mathrm{mmol})$ in anhydrous DCM $(20 \mathrm{~mL})$ was added $\mathrm{MeOH}(5 \mathrm{~mL})$ at $0{ }^{\circ} \mathrm{C}$. The mixture was stirred at $0{ }^{\circ} \mathrm{C}$ for $30 \mathrm{~min}$. A solution of compound 31e $(1.0 \mathrm{~g}, 2.86 \mathrm{mmol})$ in $\mathrm{MeOH}(10 \mathrm{~mL})$ was added via syringe at $0{ }^{\circ} \mathrm{C}$. The mixture was warmed to room temperature and stirred at this temperature for $3 \mathrm{~h}$. The solvent was removed by rotary evaporation in vacuo. The residue was washed with ether and dried under reduced pressure (oil pump) to give compound $\mathbf{3 e}(1.0 \mathrm{~g}, 98 \%)$ as its $\mathrm{HCl}$ salt.

\section{Procedures for Synthesis of Pyridoxamines 3a-c and 3g (Scheme 2)}<smiles>[R]C(=O)N[C@H]1CCc2ncc(O)c(CO)c21</smiles>

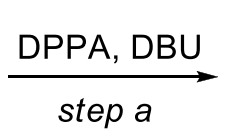

$10 \mathrm{a}-\mathrm{c}$ and $10 \mathrm{~g}$<smiles>[R]C(=O)N[C@H]1CCc2ncc(O)c(CN)c21</smiles>

$30 \mathrm{a}-\mathrm{c}$ and $30 \mathrm{~g}$<smiles>[R]C(=O)N[C@H]1CCc2ncc(O)c(CN)c21</smiles>

$3 a-c$ and $3 g$

10a, 30a, and 3a: $\mathrm{R}=n-\mathrm{Pr}$

10b, 30b, and 3b: $\mathrm{R}=\mathrm{CH}_{2} \mathrm{OCH}_{3}$

10c, 30c, and 3c: $\mathrm{R}=\mathrm{CH}_{2} \mathrm{NHAc}$

$10 \mathrm{~g}, 30 \mathrm{~g}$, and $3 \mathrm{~g}: \mathrm{R}=\mathrm{CH}_{2} \mathrm{NMe}_{2}$

Compounds 3a-c and $\mathbf{3 g}$ were prepared in $30 \%(0.075 \mathrm{~g}), 37 \%(0.10 \mathrm{~g}), 50 \%$ $(0.20 \mathrm{~g})$, and $50 \%(0.050 \mathrm{~g})$, respectively, from 10a-c $(10 a: 0.25 \mathrm{~g}, 10 \mathrm{~b}: 0.27 \mathrm{~g}, 10 \mathrm{c}$ : 
$0.40 \mathrm{~g})$ and $10 \mathrm{~g}(0.10 \mathrm{~g})$ by following a procedure similar to that applied to $\mathbf{3 e}$ but without the corresponding deprotection step.

Representative Procedure for the 3e-Catalyzed Asymmetric Transamination of $\alpha$-Keto Acids 11 (Table 2, for 13a)

To a $5 \mathrm{~mL}$ vial equipped with a magnetic stirrer bar were added 4-(naphthalen-1-yl)-2-oxobutanoic acid (11a) (0.0228 g, $0.10 \quad \mathrm{mmol})$, 2,2-diphenylglycine (12) (0.0227 g, $0.10 \mathrm{mmol})$, chiral pyridoxamine 3e (0.0072 g, $0.0020 \mathrm{mmol}), \mathrm{MeOH}(0.70 \mathrm{~mL})$, and $\mathrm{H}_{2} \mathrm{O}(0.30 \mathrm{~mL})$. After stirring at $20{ }^{\circ} \mathrm{C}$ for 6 days, the reaction mixture was transferred to a $25 \mathrm{~mL}$ round-bottom flask. $\mathrm{MeOH}$ was added until all the sold was dissolved. Then silica gel $(0.20 \mathrm{~g})$ was added. After removal of the solvent in vacuo at $30{ }^{\circ} \mathrm{C}$, the resulting residue was submitted to column chromatography on silica gel $(\mathrm{EtOH} /$ ethyl acetate / 25-28\% ammonia solution $=100: 58: 16)$ to give compound 13a $(0.0175 \mathrm{~g}, 76 \%$ yield, $70 \%$ ee $)$ as a white solid. The enantiomeric excess of 13a was deteremined by HPLC analysis after the amino acid was converted to its methyl ester by treatment with $\mathrm{CH}_{2} \mathrm{~N}_{2}$ in methanol. The enantiomeric excesses of $\mathbf{1 3 b - o}$ were deteremined by HPLC analysis after the amino acids were converted to $N$-benzoyl methyl esters by treatment with thionyl chloride in methanol and subsequent reaction with benzoyl chloride. ${ }^{6}$

\section{References}

(1) Zhu, L.; Chen, H.; Meng, Q.; Fan, W.; Xie, X.; Zhang, Z. Tetrahedron 2011, 67, 6186.

(2) Asano, Y.; Yamada, A.; Kato, Y.; Yamaguchi, K.; Hibino Y.; Hirai, K.; Kondo, K. J. Org. Chem.1990, 55, 5567.

(3) Nimitz, J. S.; Mosher, H. S. J. Org. Chem.1981, 46, 211.

(4) Nakamura, A.; Lectard, S.; Hashizume, D.; Hamashima, Y.; Sodeoka, M. J. Am. Chem. Soc.2010, 132, 4036. 
(5) Balducci, D.; Conway, P. A.; Sapuppo, G.; Müller-Bunz, H.; Paradisi, F. Tetrahedron 2012, 68, 7374.

(6) Basra, S.; Fennie, M. W.; Kozlowski, M. C. Org. Lett. 2006, 8, 2659.

\section{Characterization Data}

\section{Scheme 2, compound 3a}

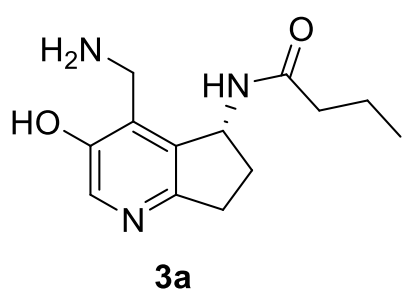

White solid; m.p. $198-200{ }^{\circ} \mathrm{C} ;[\alpha]^{25}{ }_{\mathrm{D}}=34.5$ (c 0.25, $\left.\mathrm{CH}_{3} \mathrm{OH}\right)$; IR $(\mathrm{KBr}) 3269,1636$, 1538, 1460, 1332, $1294 \mathrm{~cm}^{-1} ;{ }^{1} \mathrm{H}$ NMR (600 MHz, $\left.\mathrm{CD}_{3} \mathrm{OD}\right) \delta 7.85$ (s, 1H), 5.52-5.47 (m, 1H), $3.97(\mathrm{~d}, J=14.4 \mathrm{~Hz}, 1 \mathrm{H}), 3.92(\mathrm{~d}, J=14.4 \mathrm{~Hz}, 1 \mathrm{H}), 3.09-3.01(\mathrm{~m}, 1 \mathrm{H})$, 2.83-2.73 (m, 1H), 2.57-2.47 (m, 1H), $2.16(\mathrm{t}, J=7.2 \mathrm{~Hz}, 2 \mathrm{H}), 1.98-1.90(\mathrm{~m}, 1 \mathrm{H})$, $1.70-1.60(\mathrm{~m}, 2 \mathrm{H}), 0.95(\mathrm{t}, J=7.2 \mathrm{~Hz}, 3 \mathrm{H}) ;{ }^{13} \mathrm{C} \mathrm{NMR}\left(150 \mathrm{MHz}, \mathrm{D}_{2} \mathrm{O}\right) \delta 175.9,160.3$, 149.5, 140.2, 134.2, 127.0, 51.4, 37.6, 37.3, 31.6, 29.7, 19.0, 12.7; HRMS m/z Calcd. For $\mathrm{C}_{13} \mathrm{H}_{20} \mathrm{~N}_{3} \mathrm{O}_{2}(\mathrm{M}+\mathrm{H})^{+}:$250.1556; Found: 250.1546 .

\section{Scheme 2, compound 3b}

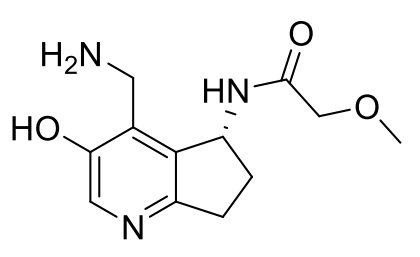

3b

White solid; m.p. $186-190{ }^{\circ} \mathrm{C}$; $[\alpha]^{25}{ }_{\mathrm{D}}=24.7$ (c 0.50, $\left.\mathrm{CH}_{3} \mathrm{OH}\right)$; IR $(\mathrm{KBr}) 1659,1537$, $1205 \mathrm{~cm}^{-1} ;{ }^{1} \mathrm{H}$ NMR $\left(600 \mathrm{MHz}, \mathrm{D}_{2} \mathrm{O}\right) \delta 8.14(\mathrm{~s}, 1 \mathrm{H}), 5.58(\mathrm{dd}, J=8.4,4.2 \mathrm{~Hz}, 1 \mathrm{H})$, $4.26(\mathrm{~d}, J=14.4 \mathrm{~Hz}, 1 \mathrm{H}), 4.15(\mathrm{~d}, J=14.4 \mathrm{~Hz}, 1 \mathrm{H}), 3.93(\mathrm{~d}, J=15.6 \mathrm{~Hz}, 1 \mathrm{H}), 3.89(\mathrm{~d}$, $J=15.6 \mathrm{~Hz}, 1 \mathrm{H}), 3.32-3.20(\mathrm{~m}, 4 \mathrm{H}), 3.11-3.03(\mathrm{~m}, 1 \mathrm{H}), 2.69-2.60(\mathrm{~m}, 1 \mathrm{H}), 2.14-2.06$ $(\mathrm{m}, 1 \mathrm{H}) ;{ }^{13} \mathrm{C} \mathrm{NMR}\left(150 \mathrm{MHz}, \mathrm{D}_{2} \mathrm{O}\right) \delta 172.0,153.8,151.1,141.7,135.8,127.3,70.8$, 58.9, 51.0, 35.4, 30.9, 28.1; HRMS $m / z$ Calcd. For $\mathrm{C}_{12} \mathrm{H}_{18} \mathrm{~N}_{3} \mathrm{O}_{3}(\mathrm{M}+\mathrm{H})^{+}:$: 252.1348; 
Found: 252.1570.

\section{Scheme 2, compound 3c}

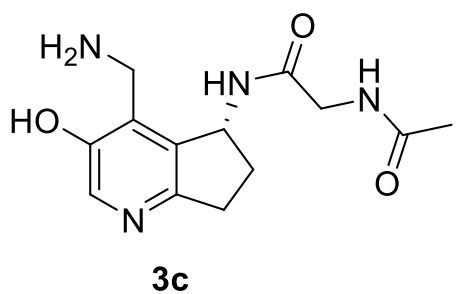

White solid; $[\alpha]_{\mathrm{D}}^{25}=56.6\left(c 0.20, \mathrm{CH}_{3} \mathrm{OH}\right) ; \mathrm{IR}(\mathrm{KBr}) 3408,3331,3285,1675,1654$, 1533, $1406 \mathrm{~cm}^{-1} ;{ }^{1} \mathrm{H}$ NMR $\left(600 \mathrm{MHz}, \mathrm{D}_{2} \mathrm{O}\right) \delta 8.25(\mathrm{~s}, 1 \mathrm{H}), 5.67(\mathrm{dd}, J=8.4,4.2 \mathrm{~Hz}$, $1 \mathrm{H}), 4.39(\mathrm{~d}, J=14.4 \mathrm{~Hz}, 1 \mathrm{H}), 4.30(\mathrm{~d}, J=14.4 \mathrm{~Hz}, 1 \mathrm{H}), 3.88(\mathrm{~s}, 2 \mathrm{H}), 3.44-3.37(\mathrm{~m}$, $1 \mathrm{H}), 3.23-3.16(\mathrm{~m}, 1 \mathrm{H}), 2.82-2.73(\mathrm{~m}, 1 \mathrm{H}), 2.26-2.20(\mathrm{~m}, 1 \mathrm{H}), 2.05(\mathrm{~s}, 3 \mathrm{H}) ;{ }^{13} \mathrm{C}$ NMR $\left(150 \mathrm{MHz}, \mathrm{D}_{2} \mathrm{O}\right) \delta 175.0,171.0,153.7,151.2,141.8,135.6,127.3,51.4,42.8,35.4$, 30.7, 28.1, 21.6; HRMS m/z Calcd. For $\mathrm{C}_{13} \mathrm{H}_{19} \mathrm{~N}_{4} \mathrm{O}_{3}(\mathrm{M}+\mathrm{H})^{+}$: 279.1457; Found: 279.1442 .

\section{Scheme 2, compound 3d}<smiles>NCC(=O)NC1CCc2ncc(O)c(CN)c21</smiles>

White solid; $[\alpha]^{25}=96.7\left(c 0.50, \mathrm{CH}_{3} \mathrm{OH}\right) ; \mathrm{IR}(\mathrm{KBr}) 3404,1679,1626,1533,1468$, 1350, $1307 \mathrm{~cm}^{-1} ;{ }^{1} \mathrm{H}$ NMR $\left(400 \mathrm{MHz}, \mathrm{D}_{2} \mathrm{O}\right) \delta 8.25(\mathrm{~s}, 1 \mathrm{H}), 5.68(\mathrm{dd}, J=8.4,3.6 \mathrm{~Hz}$, $1 \mathrm{H}), 4.44(\mathrm{~d}, J=14.4 \mathrm{~Hz}, 1 \mathrm{H}), 4.30(\mathrm{~d}, J=14.4 \mathrm{~Hz}, 1 \mathrm{H}), 3.87-3.78(\mathrm{~m}, 2 \mathrm{H})$, 3.44-3.33 (m, 1H), 3.24-3.14 (m, 1H), 2.81-2.69 (m, 1H), 2.30-2.20 (m, $1 \mathrm{H}) ;{ }^{13} \mathrm{C}$ NMR $\left(150 \mathrm{MHz}, \mathrm{D}_{2} \mathrm{O}\right) \delta 166.3,153.8,151.2,141.6,135.8,127.5,51.5,40.5,35.7$, 31.0, 28.1; HRMS $m / z$ Calcd. For $\mathrm{C}_{11} \mathrm{H}_{17} \mathrm{~N}_{4} \mathrm{O}_{2}(\mathrm{M}+\mathrm{H})^{+}$: 237.1352; Found: 237.1330. 


\section{Scheme 2, compound 3e}

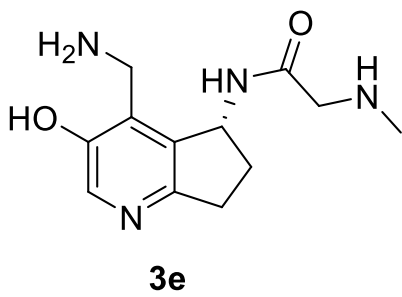

White solid; m.p. $189-193{ }^{\circ} \mathrm{C} ;[\alpha]^{25}=39.1$ (c 0.50, $\left.\mathrm{CH}_{3} \mathrm{OH}\right)$; IR (KBr) 3442, 3250, 1693, 1625, 1541, 1487, $1294 \mathrm{~cm}^{-1} ;{ }^{1} \mathrm{H}$ NMR $\left(600 \mathrm{MHz}, \mathrm{D}_{2} \mathrm{O}\right) \delta 8.29(\mathrm{~s}, 1 \mathrm{H}), 5.71(\mathrm{~d}$, $J=7.8 \mathrm{~Hz}, 1 \mathrm{H}), 4.48(\mathrm{~d}, J=14.4 \mathrm{~Hz}, 1 \mathrm{H}), 4.34(\mathrm{~d}, J=14.4 \mathrm{~Hz}, 1 \mathrm{H}), 3.98-3.88(\mathrm{~m}$, $2 \mathrm{H}), 3.48-3.36(\mathrm{~m}, 1 \mathrm{H}), 3.29-3.18(\mathrm{~m}, 1 \mathrm{H}), 2.87-2.72(\mathrm{~m}, 4 \mathrm{H}), 2.34-2.23(\mathrm{~m}, 1 \mathrm{H}) ;{ }^{13} \mathrm{C}$ NMR (150 MHz, $\left.\mathrm{D}_{2} \mathrm{O}\right) \delta 165.5,153.8,151.2,141.4,135.8,127.5,51.5,49.4,35.7$, 32.8, 30.9, 28.1; HRMS m/z Calcd. For $\mathrm{C}_{12} \mathrm{H}_{19} \mathrm{~N}_{4} \mathrm{O}_{2}(\mathrm{M}+\mathrm{H})^{+}:$251.1508; Found: 251.1489 .

\section{Scheme 2, compound 3f}

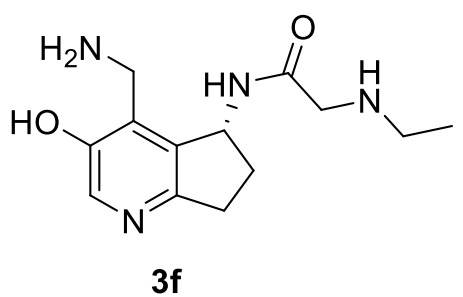

White solid; m.p. $178-180{ }^{\circ} \mathrm{C} ;[\alpha]^{25}{ }_{\mathrm{D}}=20.0\left(c\right.$ 0.30, $\left.\mathrm{CH}_{3} \mathrm{OH}\right)$; IR $(\mathrm{KBr}) 3247,1690$, 1625, 1538, $1409 \mathrm{~cm}^{-1} ;{ }^{1} \mathrm{H}$ NMR $\left(600 \mathrm{MHz}, \mathrm{D}_{2} \mathrm{O}\right) \delta 8.25(\mathrm{~s}, 1 \mathrm{H}), 5.68(\mathrm{dd}, J=8.4$, $3.6 \mathrm{~Hz}, 1 \mathrm{H}), 4.44(\mathrm{~d}, J=14.4 \mathrm{~Hz}, 1 \mathrm{H}), 4.30(\mathrm{~d}, J=14.4 \mathrm{~Hz}, 1 \mathrm{H}), 3.94-3.86(\mathrm{~m}, 2 \mathrm{H})$, 3.44-3.36 (m, 1H), 3.24-3.17 (m, 1H), 3.15 (q, $J=7.2 \mathrm{~Hz}, 2 \mathrm{H}), 2.79-2.71(\mathrm{~m}, 1 \mathrm{H})$, 2.28-2.22 (m, 1H), $1.30(\mathrm{t}, J=7.2 \mathrm{~Hz}, 3 \mathrm{H}) ;{ }^{13} \mathrm{C} \mathrm{NMR}\left(150 \mathrm{MHz}, \mathrm{D}_{2} \mathrm{O}\right) \delta 165.5,153.8$, 151.2, 141.5, 135.8, 127.5, 51.4, 47.5, 43.0, 35.7, 30.9, 28.1, 10.3; HRMS m/z Calcd. For $\mathrm{C}_{13} \mathrm{H}_{21} \mathrm{~N}_{4} \mathrm{O}_{2}(\mathrm{M}+\mathrm{H})^{+}:$265.1665; Found: 265.1637 . 


\section{Scheme 2, compound 3g}

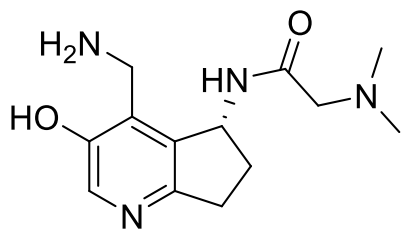

$3 g$

White solid; $[\alpha]^{25}=16.3\left(c 0.10, \mathrm{CH}_{3} \mathrm{OH}\right)$; IR (KBr) 3405, 1699, 1663, 1629, 1544, $1340 \mathrm{~cm}^{-1} ;{ }^{1} \mathrm{H}$ NMR $\left(400 \mathrm{MHz}, \mathrm{D}_{2} \mathrm{O}\right) \delta 8.22(\mathrm{~s}, 1 \mathrm{H}), 5.66(\mathrm{dd}, J=8.0,3.6 \mathrm{~Hz}, 1 \mathrm{H})$, $4.41(\mathrm{~d}, J=14.4 \mathrm{~Hz}, 1 \mathrm{H}), 4.26(\mathrm{~d}, J=14.4 \mathrm{~Hz}, 1 \mathrm{H}), 4.05(\mathrm{~d}, J=16.0 \mathrm{~Hz}, 1 \mathrm{H}), 4.00(\mathrm{~d}$, $J=16.0 \mathrm{~Hz}, 1 \mathrm{H}), 3.41-3.30(\mathrm{~m}, 1 \mathrm{H}), 3.20-3.10(\mathrm{~m}, 1 \mathrm{H}), 2.95(\mathrm{~s}, 6 \mathrm{H}), 2.78-2.67(\mathrm{~m}$, $1 \mathrm{H}), 2.28-2.18(\mathrm{~m}, 1 \mathrm{H}) ;{ }^{13} \mathrm{C}$ NMR $\left(150 \mathrm{MHz}, \mathrm{D}_{2} \mathrm{O}\right) \delta 164.5,153.8,151.5,140.8$, 135.0, 128.4, 58.0, 51.5, 43.8, 35.8, 31.0, 28.3; HRMS m/z Calcd. For $\mathrm{C}_{13} \mathrm{H}_{21} \mathrm{~N}_{4} \mathrm{O}_{2}(\mathrm{M}$ $+\mathrm{H})^{+}$: 265.1665; Found: 265.1638 .

\section{Scheme 3, compound 13b}

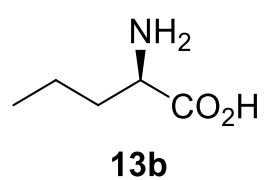

White solid, m.p. $208-212{ }^{\circ} \mathrm{C} ;[\alpha]^{25}{ }_{\mathrm{D}}=-13.0(c 0.20,1.0 \mathrm{M} \mathrm{HCl})(63 \%$ ee $)$; IR $(\mathrm{KBr})$ $1655,1582,1511,1420,1326 \mathrm{~cm}^{-1} ;{ }^{1} \mathrm{H}$ NMR $\left(400 \mathrm{MHz}, \mathrm{D}_{2} \mathrm{O}\right.$ with $\left.20 \% \mathrm{KOH}\right) \delta 2.86$ $(\mathrm{t}, J=6.4 \mathrm{~Hz}, 1 \mathrm{H}), 1.26-1.04(\mathrm{~m}, 2 \mathrm{H}), 1.00-0.86(\mathrm{~m}, 2 \mathrm{H}), 0.54(\mathrm{t}, J=7.2 \mathrm{~Hz}, 3 \mathrm{H})$.

\section{Scheme 3, compound 13c}

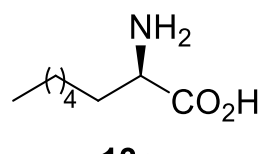

$13 c$

White solid, m.p. $225-228{ }^{\circ} \mathrm{C} ;[\alpha]^{25} \mathrm{D}=-12.7(c 0.20,1.0 \mathrm{M} \mathrm{HCl})(70 \%$ ee); IR $(\mathrm{KBr})$ 3400, 1656, 1582, 1514, 1419, $1340 \mathrm{~cm}^{-1} ;{ }^{1} \mathrm{H}$ NMR (400 MHz, $\mathrm{D}_{2} \mathrm{O}$ with $20 \% \mathrm{KOH}$ ) $\delta 2.87(\mathrm{dd}, J=6.8,6.0 \mathrm{~Hz}, 1 \mathrm{H}), 1.31-1.10(\mathrm{~m}, 2 \mathrm{H}), 1.04-0.85(\mathrm{~m}, 8 \mathrm{H}), 0.53(\mathrm{t}, J=5.6$ $\mathrm{Hz}, 3 \mathrm{H})$. 


\section{Scheme 3, compound 13d}<smiles>C/C=C/C(N)C(=O)O</smiles>

13d

White solid, m.p. $213-215{ }^{\circ} \mathrm{C} ;[\alpha]^{25}=-7.6(c 0.10,1.0 \mathrm{M} \mathrm{HCl})(69 \%$ ee $)$; IR $(\mathrm{KBr})$ 3408, 1656, 1622, 1582, 1413, 1350, $1339 \mathrm{~cm}^{-1}$; ${ }^{1} \mathrm{H}$ NMR $\left(400 \mathrm{MHz}, \mathrm{D}_{2} \mathrm{O}\right.$ with $20 \%$ $\mathrm{KOH}) \delta 2.76(\mathrm{dd}, J=7.6,5.2 \mathrm{~Hz}, 1 \mathrm{H}), 1.29-1.16(\mathrm{~m}, 1 \mathrm{H}), 1.10-0.97(\mathrm{~m}, 1 \mathrm{H})$, $0.96-0.79(\mathrm{~m}, 12 \mathrm{H}), 0.46(\mathrm{t}, J=6.4 \mathrm{~Hz}, 3 \mathrm{H})$.

\section{Scheme 3, compound 13e}<smiles>C=C[14CH]C[C@H](N)C(=O)O</smiles>

$13 e$

White solid, m.p. $202-205{ }^{\circ} \mathrm{C} ;[\alpha]^{25}{ }_{\mathrm{D}}=-1.9(c 0.10,1.0 \mathrm{M} \mathrm{HCl})(76 \%$ ee $)$; IR $(\mathrm{KBr})$ $3405,3078,1656,1582,1412 \mathrm{~cm}^{-1} ;{ }^{1} \mathrm{H}$ NMR $\left(600 \mathrm{MHz}, \mathrm{D}_{2} \mathrm{O}\right.$ with $\left.20 \% \mathrm{KOH}\right) \delta$ 5.76-5.64 (m, 1H), $4.90(\mathrm{~d}, J=16.8 \mathrm{~Hz}, 1 \mathrm{H}), 4.85(\mathrm{~d}, J=10.2 \mathrm{~Hz}, 1 \mathrm{H}), 3.09(\mathrm{dd}, J=$ 7.2, $5.4 \mathrm{~Hz}, 1 \mathrm{H}), 1.95(\mathrm{dt}, J=7.2,7.2 \mathrm{~Hz}, 2 \mathrm{H}), 1.61-1.52(\mathrm{~m}, 1 \mathrm{H}), 1.42-1.10(\mathrm{~m}, 15 \mathrm{H})$; HRMS $m / z$ Calcd. For $\mathrm{C}_{13} \mathrm{H}_{26} \mathrm{NO}_{2}(\mathrm{M}+\mathrm{H})^{+}$: 228.1964; Found: 228.1951.

\section{Scheme 3, compound $13 f$}<smiles>NC(C=COc1ccccc1)C(=O)O</smiles>

White solid, m.p. $198-200{ }^{\circ} \mathrm{C} ;[\alpha]^{25}=-2.4(c 0.10,1.0 \mathrm{M} \mathrm{HCl})(73 \%$ ee $)$; IR $(\mathrm{KBr})$ $3424,1655,1601,1585,1498,1300,1245 \mathrm{~cm}^{-1} ;{ }^{1} \mathrm{H}$ NMR $\left(600 \mathrm{MHz}, \mathrm{D}_{2} \mathrm{O}\right.$ with $20 \%$ $\mathrm{KOH}) \delta 6.74-6.67(\mathrm{~m}, 4 \mathrm{H}), 6.62(\mathrm{dd}, J=7.2,6.6 \mathrm{~Hz}, 1 \mathrm{H}), 3.83(\mathrm{~s}, 2 \mathrm{H}), 2.81(\mathrm{t}, J=$ $6.6 \mathrm{~Hz}, 2 \mathrm{H}), 2.73(\mathrm{dd}, J=7.2,6.0 \mathrm{~Hz}, 1 \mathrm{H}), 1.21-1.13(\mathrm{~m}, 1 \mathrm{H}), 1.05-0.94(\mathrm{~m}, 3 \mathrm{H})$, 0.85-0.72 (m, 6H). 


\section{Scheme 3, compound 13g}<smiles>NC(CCc1ccccc1-c1ccccc1)C(=O)O</smiles>

13g:

White solid, m.p. $223-225{ }^{\circ} \mathrm{C} ;[\alpha]^{25}{ }_{\mathrm{D}}=-3.2(c 0.30,1.0 \mathrm{M} \mathrm{HCl})(71 \%$ ee $)$; IR $(\mathrm{KBr})$ 3407, 1665, 1632, 1575, 1510, $1411 \mathrm{~cm}^{-1}$; ${ }^{1} \mathrm{H}$ NMR (600 MHz, $\mathrm{D}_{2} \mathrm{O}$ with 2.0 equiv. of $\mathrm{KOH}) \delta 7.45(\mathrm{dd}, J=7.8,7.2 \mathrm{~Hz}, 2 \mathrm{H}), 7.42-7.24(\mathrm{~m}, 6 \mathrm{H}), 7.21(\mathrm{~d}, J=7.2 \mathrm{~Hz}, 1 \mathrm{H})$, $3.01(\mathrm{t}, J=6.0 \mathrm{~Hz}, 1 \mathrm{H}), 2.54(\mathrm{t}, J=8.4 \mathrm{~Hz}, 2 \mathrm{H}), 1.72-1.63(\mathrm{~m}, 1 \mathrm{H}), 1.61-1.50(\mathrm{~m}$, $1 \mathrm{H})$.

\section{Scheme 3, compound 13h}<smiles>Cc1cc(C)c(CC[C@H](N)C(=O)O)c(C)c1</smiles>

$13 \mathrm{~h}$

White solid, m.p. $234-236{ }^{\circ} \mathrm{C} ;[\alpha]^{25}{ }_{\mathrm{D}}=-22.5(c 0.10,1.0 \mathrm{M} \mathrm{HCl})(87 \%$ ee $)$; IR $(\mathrm{KBr})$ $3435,1624,1593,1520,1407 \mathrm{~cm}^{-1} ;{ }^{1} \mathrm{H}$ NMR (600 MHz, $\mathrm{D}_{2} \mathrm{O}$ with $\left.20 \% \mathrm{KOH}\right) \delta 6.55$ $(\mathrm{s}, 2 \mathrm{H}), 2.96(\mathrm{t}, J=6.0 \mathrm{~Hz}, 1 \mathrm{H}), 2.26-2.16(\mathrm{~m}, 2 \mathrm{H}), 1.89(\mathrm{~s}, 6 \mathrm{H}), 1.85(\mathrm{~s}, 3 \mathrm{H})$, $1.34-1.23(\mathrm{~m}, 2 \mathrm{H})$

\section{Scheme 3, compound 13a}<smiles>NC(CCc1cccc2ccccc12)C(=O)O</smiles>

$13 a$

White solid, m.p. $211-214{ }^{\circ} \mathrm{C} ;[\alpha]^{25} \mathrm{D}=-14.0(c 0.20,1.0 \mathrm{M} \mathrm{HCl})(70 \%$ ee $)$; IR $(\mathrm{KBr})$ 3423, 1595, 1479, 1403, $1349 \mathrm{~cm}^{-1}$; ${ }^{1} \mathrm{H}$ NMR (600 MHz, $\mathrm{D}_{2} \mathrm{O}$ with $\left.20 \% \mathrm{KOH}\right) \delta 7.89$ $(\mathrm{d}, J=8.4 \mathrm{~Hz}, 1 \mathrm{H}), 7.68(\mathrm{~d}, J=7.8 \mathrm{~Hz}, 1 \mathrm{H}), 7.54(\mathrm{~d}, J=7.8 \mathrm{~Hz}, 1 \mathrm{H}), 7.39(\mathrm{dd}, J=$ 7.8, 7.2 Hz, 1H), $7.33(\mathrm{dd}, J=7.8,7.2 \mathrm{~Hz}, 1 \mathrm{H}), 7.24(\mathrm{t}, J=7.8,7.2 \mathrm{~Hz}, 1 \mathrm{H}), 7.19$ (d, $J=7.2 \mathrm{~Hz}, 1 \mathrm{H}), 3.14(\mathrm{dd}, J=6.6,6.0 \mathrm{~Hz}, 1 \mathrm{H}), 2.90-2.82(\mathrm{~m}, 2 \mathrm{H}), 1.83-1.74(\mathrm{~m}, 1 \mathrm{H})$, $1.73-1.63(\mathrm{~m}, 1 \mathrm{H})$ 


\section{Scheme 3, compound 13i}<smiles>N[C@@H](CCc1ccc2c(c1)CCO2)C(=O)O</smiles>

$13 \mathbf{i}$

White solid, m.p. $235-237{ }^{\circ} \mathrm{C} ;[\alpha]^{25}{ }_{\mathrm{D}}=-11.9(c 0.20,1.0 \mathrm{M} \mathrm{HCl})(81 \%$ ee $)$; IR $(\mathrm{KBr})$ 3419, 1657, 1603, 1583, 1518, 1493, $1407 \mathrm{~cm}^{-1} ;{ }^{1} \mathrm{H}$ NMR $\left(600 \mathrm{MHz}, \mathrm{D}_{2} \mathrm{O}\right.$ with $20 \%$ KOH) $\delta 6.60(\mathrm{~s}, 1 \mathrm{H}), 6.50(\mathrm{~d}, J=7.8 \mathrm{~Hz}, 1 \mathrm{H}), 6.25(\mathrm{~d}, J=7.8 \mathrm{~Hz}, 1 \mathrm{H}), 3.99(\mathrm{t}, J=$ $9.0 \mathrm{~Hz}, 2 \mathrm{H}), 2.75(\mathrm{dd}, J=6.6,6.0 \mathrm{~Hz}, 1 \mathrm{H}), 2.60(\mathrm{t}, J=9.0 \mathrm{~Hz}, 2 \mathrm{H}), 2.13-2.00(\mathrm{~m}$, 2H), 1.42-1.34 (m, 1H), 1.29-1.20 (m, 1H); HRMS m/z Calcd. For $\mathrm{C}_{12} \mathrm{H}_{16} \mathrm{NO}_{3}(\mathrm{M}+$ $\mathrm{H})^{+}:$222.1130; Found: 222.1094.

\section{Scheme 3, compound 13j}<smiles>N[C@@H](Cc1ccccc1)C(=O)O</smiles>

13j

White solid, m.p. $207-211{ }^{\circ} \mathrm{C} ;[\alpha]^{25}=9.3(c 0.10,1.0 \mathrm{M} \mathrm{HCl})(66 \%$ ee $)$; IR $(\mathrm{KBr})$ $3434,1609,1509,1394 \mathrm{~cm}^{-1} ;{ }^{1} \mathrm{H}$ NMR $\left(600 \mathrm{MHz}, \mathrm{D}_{2} \mathrm{O}\right.$ with $\left.20 \% \mathrm{KOH}\right) \delta 7.22(\mathrm{dd}, J$ $=7.8,7.2 \mathrm{~Hz}, 2 \mathrm{H}), 7.15(\mathrm{dd}, J=7.8,7.2 \mathrm{~Hz}, 1 \mathrm{H}), 7.11(\mathrm{~d}, J=7.2 \mathrm{~Hz}, 2 \mathrm{H}), 3.33(\mathrm{dd}, J$ $=7.2,6.0 \mathrm{~Hz}, 1 \mathrm{H}), 2.82(\mathrm{dd}, J=13.2,6.0 \mathrm{~Hz}, 1 \mathrm{H}), 2.67(\mathrm{dd}, J=13.2,7.2 \mathrm{~Hz}, 1 \mathrm{H})$.

\section{Scheme 3, compound 13k}<smiles>N[C@@H](Cc1ccc2ccccc2c1)C(=O)O</smiles>

White solid, m.p. $221-224{ }^{\circ} \mathrm{C} ;[\alpha]^{25} \mathrm{D}=6.4(c 0.30,1.0 \mathrm{M} \mathrm{HCl})(68 \%$ ee $)$; IR $(\mathrm{KBr})$ 3446, 3052, 1616, 1586, 1504, 1409, $1313 \mathrm{~cm}^{-1}$; ${ }^{1} \mathrm{H}$ NMR $\left(600 \mathrm{MHz}, \mathrm{D}_{2} \mathrm{O}\right.$ with $20 \%$ $\mathrm{KOH}) \delta 7.34(\mathrm{~d}, J=7.8 \mathrm{~Hz}, 1 \mathrm{H}), 7.30-7.24(\mathrm{~m}, 2 \mathrm{H}), 7.20(\mathrm{~s}, 1 \mathrm{H}), 6.99(\mathrm{dd}, J=7.8$, $7.2 \mathrm{~Hz}, 1 \mathrm{H}), 6.97-6.90(\mathrm{~m}, 2 \mathrm{H}), 3.18(\mathrm{dd}, J=8.4,4.8 \mathrm{~Hz}, 1 \mathrm{H}), 2.81(\mathrm{dd}, J=13.8,4.8$ $\mathrm{Hz}, 1 \mathrm{H}), 2.48(\mathrm{dd}, J=13.8,8.4 \mathrm{~Hz}, 1 \mathrm{H})$. 


\section{Scheme 3, compound 131}<smiles>CC(C)C[C@H](N)C(=O)O</smiles>

131

White solid, m.p. $232-234{ }^{\circ} \mathrm{C} ;[\alpha]^{25}{ }_{\mathrm{D}}=-14.3(c 0.10,1.0 \mathrm{M} \mathrm{HCl})(64 \%$ ee $)$; IR $(\mathrm{KBr})$ 3424, 3103, 1617, 1587, 1514, 1415, 1340, 1312, $1296 \mathrm{~cm}^{-1}$; ${ }^{1} \mathrm{H}$ NMR (600 MHz, $\mathrm{D}_{2} \mathrm{O}$ with $\left.20 \% \mathrm{KOH}\right) \delta 3.08(\mathrm{dd}, J=8.4,6.0 \mathrm{~Hz}, 1 \mathrm{H}), 1.54-1.44(\mathrm{~m}, 1 \mathrm{H}), 1.32-1.26$ (m, 1H), 1.23-1.16 (m, 1H), $0.75(\mathrm{~d}, J=7.2 \mathrm{~Hz}, 3 \mathrm{H}), 0.73(\mathrm{~d}, J=6.6 \mathrm{~Hz}, 3 \mathrm{H})$.

\section{Scheme 3, compound 13m}

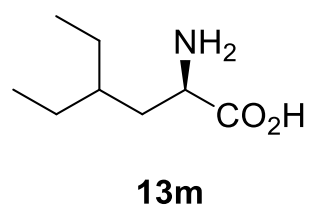

White solid, m.p. $220-222{ }^{\circ} \mathrm{C} ;[\alpha]^{25}{ }_{\mathrm{D}}=-3.6(c 0.10,1.0 \mathrm{M} \mathrm{HCl})(70 \%$ ee $)$; IR $(\mathrm{KBr})$ $3411,3038,1625,1590,1523,1405 \mathrm{~cm}^{-1} ;{ }^{1} \mathrm{H}$ NMR (400 MHz, $\mathrm{D}_{2} \mathrm{O}$ with $20 \% \mathrm{KOH}$ ) $\delta 2.84(\mathrm{dd}, J=8.4,6.0 \mathrm{~Hz}, 1 \mathrm{H}), 1.16-1.07(\mathrm{~m}, 1 \mathrm{H}), 1.02-0.82(\mathrm{~m}, 6 \mathrm{H}), 0.43(\mathrm{t}, J=6.8$ $\mathrm{Hz}, 6 \mathrm{H})$.

\section{Scheme 3, compound 13n}<smiles>NC(CC(c1ccccc1)c1ccccc1)C(=O)O</smiles>

$13 n$

White solid, m.p. $207-209{ }^{\circ} \mathrm{C} ;[\alpha]^{25}{ }_{\mathrm{D}}=-4.9(c 0.10,1.0 \mathrm{M} \mathrm{HCl})(78 \%$ ee $)$; IR $(\mathrm{KBr})$ $3607,3474,3027,1633,1598,1535,1493,1400 \mathrm{~cm}^{-1}$; ${ }^{1} \mathrm{H}$ NMR $\left(600 \mathrm{MHz}, \mathrm{D}_{2} \mathrm{O}\right.$ with $20 \% \mathrm{KOH}) \delta 7.30-7.19(\mathrm{~m}, 8 \mathrm{H}), 7.15-7.08(\mathrm{~m}, 2 \mathrm{H}), 4.00(\mathrm{dd}, J=9.0,7.2 \mathrm{~Hz}, 1 \mathrm{H})$, $2.91(\mathrm{dd}, J=8.4,5.4 \mathrm{~Hz}, 1 \mathrm{H}), 2.39-2.31(\mathrm{~m}, 1 \mathrm{H}), 2.06-1.99(\mathrm{~m}, 1 \mathrm{H})$. 


\section{Scheme 3, compound 130}

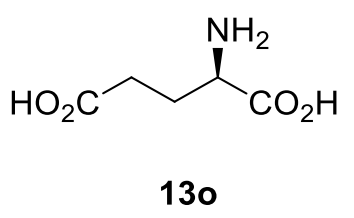

White solid, m.p. $200-202{ }^{\circ} \mathrm{C} ;[\alpha]^{25}=-1.6(c 0.20,1.0 \mathrm{M} \mathrm{HCl})(14 \%$ ee $)$; IR ( $\left.\mathrm{KBr}\right)$ 3392, 3216, 3039, 1703, 1633, 1566, $1498 \mathrm{~cm}^{-1}$; ${ }^{1} \mathrm{H}$ NMR (400 MHz, $\mathrm{D}_{2} \mathrm{O}$ with $20 \%$ $\mathrm{KOH}) \delta 2.86(\mathrm{dd}, J=6.8,6.0 \mathrm{~Hz}, 1 \mathrm{H}), 1.84(\mathrm{t}, J=8.4 \mathrm{~Hz}, 2 \mathrm{H}), 1.57-1.44(\mathrm{~m}, 1 \mathrm{H})$, $1.44-1.33(\mathrm{~m}, 1 \mathrm{H})$. 
The X-ray structure of compound $(S, R)-8$

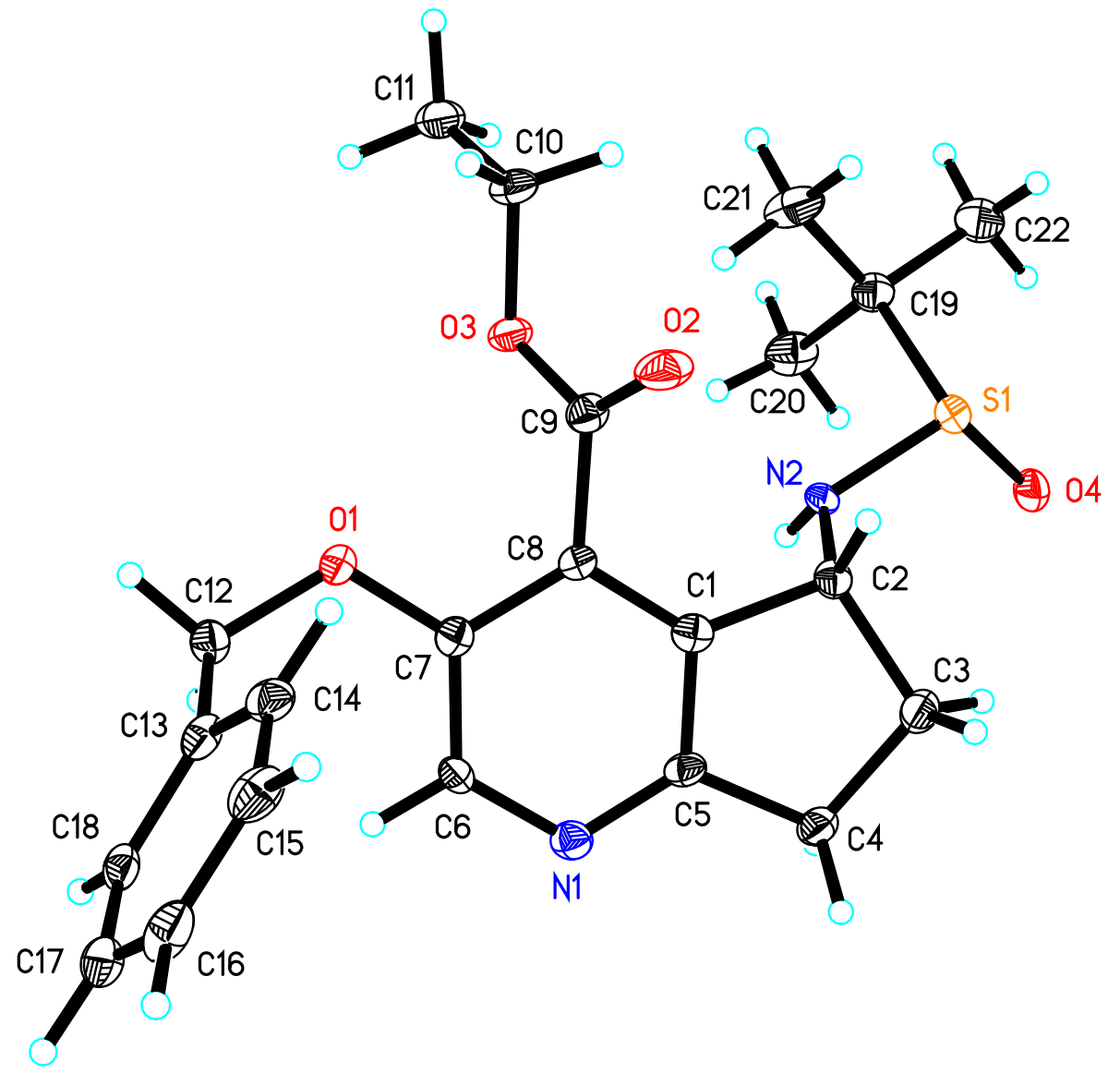


Table 1. Crystal data and structure refinement for mo_(S,R)-8_0m.

Identification code

Empirical formula

Formula weight

Temperature

Wavelength

Crystal system

Space group

Unit cell dimensions

$108.916(4)^{\circ}$

Volume

Z

Density (calculated)

Absorption coefficient

$\mathrm{F}(000)$

Crystal size

Theta range for data collection

Index ranges

Reflections collected

Independent reflections

Completeness to theta $=25.242^{\circ}$

Absorption correction

Max. and min. transmission

Refinement method

Data / restraints / parameters

Goodness-of-fit on $\mathrm{F}^{2}$

Final R indices [I $>2 \operatorname{sigma}(\mathrm{I})]$

$\mathrm{R}$ indices (all data)

Absolute structure parameter

Extinction coefficient

Largest diff. peak and hole
mo_(S,R)-8_0m

C44 H58 N4 O9 S2

851.06

133(2) K

$0.71073 \AA$

Monoclinic

P 21

$\mathrm{a}=8.0776(15) \AA \quad \mathrm{a}=90^{\circ}$.

$\mathrm{b}=24.432(5) \AA \quad \mathrm{b}=$

$\mathrm{c}=11.904(2) \AA \quad \mathrm{g}=90^{\circ}$.

2222.4(7) $\AA^{3}$

2

$1.272 \mathrm{Mg} / \mathrm{m}^{3}$

$0.178 \mathrm{~mm}^{-1}$

908

$0.211 \times 0.165 \times 0.123 \mathrm{~mm}^{3}$

1.667 to $25.499^{\circ}$.

$-9<=\mathrm{h}<=9,-29<=\mathrm{k}<=28,-14<=\mathrm{l}<=14$

15414

$8126[\mathrm{R}($ int $)=0.0448]$

$99.7 \%$

Semi-empirical from equivalents

0.7456 and 0.4978

Full-matrix least-squares on $\mathrm{F}^{2}$

$8126 / 3 / 557$

1.053

$\mathrm{R} 1=0.0684, \mathrm{wR} 2=0.1828$

$\mathrm{R} 1=0.0772, \mathrm{wR} 2=0.1903$

$0.03(5)$

$0.007(2)$

0.862 and -0.317 e. $\AA^{-3}$ 
Table 2. Atomic coordinates ( $\left.\quad 10^{4}\right)$ and equivalent isotropic displacement parameters $\left(\AA^{2} \mathrm{x} 10^{3}\right)$ for mo_(S,R)-8_0m. U(eq) is defined as one third of the trace of the orthogonalized $\mathrm{U}^{\mathrm{ij}}$ tensor.

\begin{tabular}{|c|c|c|c|c|}
\hline & $\mathrm{X}$ & $\mathrm{y}$ & $\mathrm{z}$ & $\mathrm{U}(\mathrm{eq})$ \\
\hline$S(1)$ & $11692(2)$ & $5140(1)$ & $10645(1)$ & $26(1)$ \\
\hline$S(2)$ & $5975(2)$ & $7852(1)$ & $5201(2)$ & $29(1)$ \\
\hline $\mathrm{N}(1)$ & $5690(7)$ & $4143(2)$ & $6813(5)$ & $28(1)$ \\
\hline $\mathrm{N}(2)$ & $10196(7)$ & $4978(2)$ & $9370(5)$ & $22(1)$ \\
\hline $\mathrm{N}(3)$ & $8495(8)$ & $8969(2)$ & $1508(5)$ & $26(1)$ \\
\hline $\mathrm{N}(4)$ & $6247(8)$ & $8071(2)$ & $3958(5)$ & $22(1)$ \\
\hline $\mathrm{O}(1)$ & $5186(6)$ & $5473(2)$ & $5323(4)$ & $30(1)$ \\
\hline $\mathrm{O}(2)$ & $6779(9)$ & $6054(2)$ & $8373(5)$ & $44(2)$ \\
\hline $\mathrm{O}(3)$ & $8075(7)$ & $6049(2)$ & $6980(4)$ & $29(1)$ \\
\hline $\mathrm{O}(4)$ & $12630(7)$ & $4638(2)$ & $11249(4)$ & $33(1)$ \\
\hline $\mathrm{O}(5)$ & $7128(7)$ & $7716(2)$ & $-211(4)$ & $32(1)$ \\
\hline $\mathrm{O}(6)$ & $5901(7)$ & $7054(2)$ & $1463(5)$ & $38(1)$ \\
\hline $\mathrm{O}(7)$ & $8656(7)$ & $7065(2)$ & $2723(5)$ & $36(1)$ \\
\hline $\mathrm{O}(8)$ & $5674(8)$ & $8324(3)$ & $5904(5)$ & $45(2)$ \\
\hline $\mathrm{O}(9)$ & $4437(9)$ & $9125(3)$ & $7121(7)$ & $63(2)$ \\
\hline $\mathrm{C}(1)$ & $7222(9)$ & $4836(3)$ & $8147(6)$ & $25(1)$ \\
\hline $\mathrm{C}(2)$ & $8431(9)$ & $4886(3)$ & $9412(6)$ & $25(1)$ \\
\hline$C(3)$ & $8128(10)$ & $4332(3)$ & 9981(6) & $32(2)$ \\
\hline$C(4)$ & $7324(10)$ & $3930(3)$ & $8927(6)$ & $31(2)$ \\
\hline$C(5)$ & $6672(9)$ & $4304(3)$ & $7882(6)$ & $24(1)$ \\
\hline$C(6)$ & $5207(9)$ & $4526(3)$ & $5955(6)$ & $26(1)$ \\
\hline$C(7)$ & $5687(8)$ & $5072(3)$ & $6157(6)$ & $25(1)$ \\
\hline $\mathrm{C}(8)$ & $6725(8)$ & $5235(3)$ & $7291(5)$ & $22(1)$ \\
\hline $\mathrm{C}(9)$ & $7179(9)$ & $5821(3)$ & $7607(6)$ & $27(2)$ \\
\hline$C(10)$ & $8526(11)$ & $6625(3)$ & $7244(7)$ & $34(2)$ \\
\hline $\mathrm{C}(11)$ & $9740(12)$ & $6791(3)$ & $6585(7)$ & $40(2)$ \\
\hline $\mathrm{C}(12)$ & 3931(9) & $5337(3)$ & $4198(6)$ & $30(2)$ \\
\hline$C(13)$ & 2124(9) & $5235(3)$ & $4253(6)$ & $28(2)$ \\
\hline$C(14)$ & $1485(11)$ & $5509(3)$ & $5047(7)$ & $32(2)$ \\
\hline$C(15)$ & $-207(11)$ & $5428(3)$ & $5039(8)$ & $38(2)$ \\
\hline
\end{tabular}




\begin{tabular}{|c|c|c|c|c|}
\hline$C(16)$ & $-1285(10)$ & $5058(3)$ & $4229(7)$ & $39(2)$ \\
\hline$C(17)$ & $-648(10)$ & $4785(3)$ & $3446(7)$ & $36(2)$ \\
\hline$C(18)$ & $1028(10)$ & $4861(3)$ & $3455(6)$ & $28(2)$ \\
\hline$C(19)$ & $13251(10)$ & $5503(3)$ & $10072(7)$ & $34(2)$ \\
\hline$C(20)$ & $13751(11)$ & $5156(4)$ & $9182(8)$ & $43(2)$ \\
\hline$C(21)$ & $12374(13)$ & $6019(4)$ & $9502(10)$ & $52(2)$ \\
\hline $\mathrm{C}(22)$ & $14813(13)$ & $5623(4)$ & $11156(8)$ & $54(2)$ \\
\hline$C(23)$ & $8045(8)$ & $8250(3)$ & $2731(6)$ & $21(1)$ \\
\hline $\mathrm{C}(24)$ & $8051(9)$ & $8162(3)$ & $3997(5)$ & $23(1)$ \\
\hline$C(25)$ & $8959(10)$ & $8689(3)$ & $4628(6)$ & $30(2)$ \\
\hline$C(26)$ & $8943(10)$ & $9115(3)$ & $3668(6)$ & $29(2)$ \\
\hline $\mathrm{C}(27)$ & $8473(9)$ & $8777(3)$ & $2561(6)$ & $24(1)$ \\
\hline $\mathrm{C}(28)$ & $8033(10)$ & $8619(3)$ & $598(6)$ & $29(2)$ \\
\hline$C(29)$ & 7572(9) & $8076(3)$ & $687(6)$ & $24(1)$ \\
\hline$C(30)$ & $7589(8)$ & $7883(3)$ & $1799(5)$ & $24(1)$ \\
\hline $\mathrm{C}(31)$ & $7226(9)$ & $7297(3)$ & $1936(6)$ & $25(1)$ \\
\hline$C(32)$ & $8621(12)$ & $6471(3)$ & $2868(8)$ & $40(2)$ \\
\hline $\mathrm{C}(33)$ & $9267(12)$ & $6203(3)$ & 1932(8) & $46(2)$ \\
\hline$C(34)$ & $7341(9)$ & $7873(3)$ & $-1323(5)$ & $30(2)$ \\
\hline$C(35)$ & $9218(9)$ & 7907(3) & $-1267(6)$ & $28(1)$ \\
\hline$C(36)$ & $9703(10)$ & $8250(3)$ & $-2054(6)$ & $30(2)$ \\
\hline $\mathrm{C}(37)$ & $11375(11)$ & $8262(3)$ & $-2063(6)$ & $36(2)$ \\
\hline $\mathrm{C}(38)$ & $12657(11)$ & $7950(3)$ & $-1274(8)$ & $40(2)$ \\
\hline C(39) & $12207(11)$ & $7608(4)$ & $-478(7)$ & $45(2)$ \\
\hline$C(40)$ & $10508(10)$ & 7594(3) & $-471(7)$ & $35(2)$ \\
\hline $\mathrm{C}(41)$ & $3818(10)$ & $7511(4)$ & $4550(7)$ & $37(2)$ \\
\hline$C(42)$ & $3241(12)$ & $7376(5)$ & $5631(8)$ & $52(2)$ \\
\hline$C(43)$ & $4154(12)$ & $6999(4)$ & $3945(9)$ & $50(2)$ \\
\hline$C(44)$ & $2545(10)$ & $7898(4)$ & $3714(8)$ & $46(2)$ \\
\hline
\end{tabular}


Table 3. Bond lengths $[\AA ̊ \cap]$ and angles $\left[{ }^{\circ}\right]$ for mo_(S,R)-8_0m.

\begin{tabular}{|c|c|}
\hline $\mathrm{S}(1)-\mathrm{O}(4)$ & $1.497(5)$ \\
\hline $\mathrm{S}(1)-\mathrm{N}(2)$ & $1.653(6)$ \\
\hline$S(1)-C(19)$ & $1.843(8)$ \\
\hline $\mathrm{S}(2)-\mathrm{O}(8)$ & $1.489(6)$ \\
\hline $\mathrm{S}(2)-\mathrm{N}(4)$ & $1.653(5)$ \\
\hline$S(2)-C(41)$ & $1.859(8)$ \\
\hline $\mathrm{N}(1)-\mathrm{C}(5)$ & $1.323(9)$ \\
\hline $\mathrm{N}(1)-\mathrm{C}(6)$ & $1.346(9)$ \\
\hline $\mathrm{N}(2)-\mathrm{C}(2)$ & $1.460(9)$ \\
\hline $\mathrm{N}(2)-\mathrm{H}(2 \mathrm{~A})$ & $0.83(9)$ \\
\hline $\mathrm{N}(3)-\mathrm{C}(28)$ & $1.336(9)$ \\
\hline $\mathrm{N}(3)-\mathrm{C}(27)$ & $1.344(9)$ \\
\hline $\mathrm{N}(4)-\mathrm{C}(24)$ & $1.460(9)$ \\
\hline $\mathrm{N}(4)-\mathrm{H}(4)$ & $0.88(7)$ \\
\hline $\mathrm{O}(1)-\mathrm{C}(7)$ & $1.361(8)$ \\
\hline $\mathrm{O}(1)-\mathrm{C}(12)$ & $1.433(9)$ \\
\hline $\mathrm{O}(2)-\mathrm{C}(9)$ & $1.204(8)$ \\
\hline $\mathrm{O}(3)-\mathrm{C}(9)$ & $1.318(8)$ \\
\hline $\mathrm{O}(3)-\mathrm{C}(10)$ & $1.462(9)$ \\
\hline $\mathrm{O}(5)-\mathrm{C}(29)$ & $1.340(8)$ \\
\hline $\mathrm{O}(5)-\mathrm{C}(34)$ & $1.440(8)$ \\
\hline $\mathrm{O}(6)-\mathrm{C}(31)$ & $1.195(9)$ \\
\hline $\mathrm{O}(7)-\mathrm{C}(31)$ & $1.353(9)$ \\
\hline $\mathrm{O}(7)-\mathrm{C}(32)$ & $1.463(9)$ \\
\hline $\mathrm{O}(9)-\mathrm{H}(9 \mathrm{~A})$ & $0.82(3)$ \\
\hline $\mathrm{O}(9)-\mathrm{H}(9 \mathrm{~B})$ & $0.83(3)$ \\
\hline $\mathrm{C}(1)-\mathrm{C}(8)$ & $1.373(9)$ \\
\hline$C(1)-C(5)$ & $1.376(9)$ \\
\hline$C(1)-C(2)$ & $1.510(9)$ \\
\hline$C(2)-C(3)$ & $1.569(10)$ \\
\hline $\mathrm{C}(2)-\mathrm{H}(2)$ & 1.0000 \\
\hline$C(3)-C(4)$ & $1.558(10)$ \\
\hline $\mathrm{C}(3)-\mathrm{H}(3 \mathrm{~A})$ & 0.9900 \\
\hline $\mathrm{C}(3)-\mathrm{H}(3 \mathrm{~B})$ & 0.9900 \\
\hline$C(4)-C(5)$ & $1.495(9)$ \\
\hline $\mathrm{C}(4)-\mathrm{H}(4 \mathrm{C})$ & 0.9900 \\
\hline
\end{tabular}




\begin{tabular}{|c|c|}
\hline $\mathrm{C}(4)-\mathrm{H}(4 \mathrm{D})$ & 0.9900 \\
\hline$C(6)-C(7)$ & $1.389(10)$ \\
\hline $\mathrm{C}(6)-\mathrm{H}(6)$ & 0.9500 \\
\hline $\mathrm{C}(7)-\mathrm{C}(8)$ & $1.396(9)$ \\
\hline C(8)-C(9) & $1.496(9)$ \\
\hline $\mathrm{C}(10)-\mathrm{C}(11)$ & $1.497(11)$ \\
\hline $\mathrm{C}(10)-\mathrm{H}(10 \mathrm{~A})$ & 0.9900 \\
\hline $\mathrm{C}(10)-\mathrm{H}(10 \mathrm{~B})$ & 0.9900 \\
\hline $\mathrm{C}(11)-\mathrm{H}(11 \mathrm{~A})$ & 0.9800 \\
\hline $\mathrm{C}(11)-\mathrm{H}(11 \mathrm{~B})$ & 0.9800 \\
\hline $\mathrm{C}(11)-\mathrm{H}(11 \mathrm{C})$ & 0.9800 \\
\hline$C(12)-C(13)$ & $1.503(10)$ \\
\hline $\mathrm{C}(12)-\mathrm{H}(12 \mathrm{~A})$ & 0.9900 \\
\hline $\mathrm{C}(12)-\mathrm{H}(12 \mathrm{~B})$ & 0.9900 \\
\hline $\mathrm{C}(13)-\mathrm{C}(14)$ & $1.387(10)$ \\
\hline $\mathrm{C}(13)-\mathrm{C}(18)$ & $1.405(10)$ \\
\hline $\mathrm{C}(14)-\mathrm{C}(15)$ & $1.378(12)$ \\
\hline $\mathrm{C}(14)-\mathrm{H}(14)$ & 0.9500 \\
\hline$C(15)-C(16)$ & $1.400(13)$ \\
\hline $\mathrm{C}(15)-\mathrm{H}(15)$ & 0.9500 \\
\hline$C(16)-C(17)$ & $1.375(11)$ \\
\hline $\mathrm{C}(16)-\mathrm{H}(16)$ & 0.9500 \\
\hline $\mathrm{C}(17)-\mathrm{C}(18)$ & $1.363(11)$ \\
\hline $\mathrm{C}(17)-\mathrm{H}(17)$ & 0.9500 \\
\hline $\mathrm{C}(18)-\mathrm{H}(18)$ & 0.9500 \\
\hline$C(19)-C(21)$ & $1.497(12)$ \\
\hline$C(19)-C(20)$ & $1.511(11)$ \\
\hline$C(19)-C(22)$ & $1.512(12)$ \\
\hline $\mathrm{C}(20)-\mathrm{H}(20 \mathrm{~A})$ & 0.9800 \\
\hline $\mathrm{C}(20)-\mathrm{H}(20 \mathrm{~B})$ & 0.9800 \\
\hline $\mathrm{C}(20)-\mathrm{H}(20 \mathrm{C})$ & 0.9800 \\
\hline $\mathrm{C}(21)-\mathrm{H}(21 \mathrm{~A})$ & 0.9800 \\
\hline $\mathrm{C}(21)-\mathrm{H}(21 \mathrm{~B})$ & 0.9800 \\
\hline $\mathrm{C}(21)-\mathrm{H}(21 \mathrm{C})$ & 0.9800 \\
\hline $\mathrm{C}(22)-\mathrm{H}(22 \mathrm{~A})$ & 0.9800 \\
\hline $\mathrm{C}(22)-\mathrm{H}(22 \mathrm{~B})$ & 0.9800 \\
\hline $\mathrm{C}(22)-\mathrm{H}(22 \mathrm{C})$ & 0.9800 \\
\hline $\mathrm{C}(23)-\mathrm{C}(27)$ & $1.365(9)$ \\
\hline
\end{tabular}




\begin{tabular}{|c|c|}
\hline $\mathrm{C}(23)-\mathrm{C}(30)$ & $1.380(9)$ \\
\hline $\mathrm{C}(23)-\mathrm{C}(24)$ & $1.521(9)$ \\
\hline$C(24)-C(25)$ & $1.551(9)$ \\
\hline $\mathrm{C}(24)-\mathrm{H}(24)$ & 1.0000 \\
\hline$C(25)-C(26)$ & $1.543(9)$ \\
\hline $\mathrm{C}(25)-\mathrm{H}(25 \mathrm{~A})$ & 0.9900 \\
\hline $\mathrm{C}(25)-\mathrm{H}(25 \mathrm{~B})$ & 0.9900 \\
\hline$C(26)-C(27)$ & $1.495(9)$ \\
\hline $\mathrm{C}(26)-\mathrm{H}(26 \mathrm{~A})$ & 0.9900 \\
\hline $\mathrm{C}(26)-\mathrm{H}(26 \mathrm{~B})$ & 0.9900 \\
\hline $\mathrm{C}(28)-\mathrm{C}(29)$ & $1.391(10)$ \\
\hline $\mathrm{C}(28)-\mathrm{H}(28)$ & 0.9500 \\
\hline C(29)-C(30) & $1.402(9)$ \\
\hline $\mathrm{C}(30)-\mathrm{C}(31)$ & $1.479(10)$ \\
\hline $\mathrm{C}(32)-\mathrm{C}(33)$ & $1.524(12)$ \\
\hline $\mathrm{C}(32)-\mathrm{H}(32 \mathrm{~A})$ & 0.9900 \\
\hline $\mathrm{C}(32)-\mathrm{H}(32 \mathrm{~B})$ & 0.9900 \\
\hline $\mathrm{C}(33)-\mathrm{H}(33 \mathrm{~A})$ & 0.9800 \\
\hline $\mathrm{C}(33)-\mathrm{H}(33 \mathrm{~B})$ & 0.9800 \\
\hline $\mathrm{C}(33)-\mathrm{H}(33 \mathrm{C})$ & 0.9800 \\
\hline $\mathrm{C}(34)-\mathrm{C}(35)$ & $1.498(10)$ \\
\hline $\mathrm{C}(34)-\mathrm{H}(34 \mathrm{~A})$ & 0.9900 \\
\hline $\mathrm{C}(34)-\mathrm{H}(34 \mathrm{~B})$ & 0.9900 \\
\hline $\mathrm{C}(35)-\mathrm{C}(40)$ & $1.389(10)$ \\
\hline$C(35)-C(36)$ & $1.404(10)$ \\
\hline$C(36)-C(37)$ & $1.354(11)$ \\
\hline $\mathrm{C}(36)-\mathrm{H}(36)$ & 0.9500 \\
\hline $\mathrm{C}(37)-\mathrm{C}(38)$ & $1.381(12)$ \\
\hline $\mathrm{C}(37)-\mathrm{H}(37)$ & 0.9500 \\
\hline $\mathrm{C}(38)-\mathrm{C}(39)$ & $1.396(13)$ \\
\hline $\mathrm{C}(38)-\mathrm{H}(38)$ & 0.9500 \\
\hline C(39)-C(40) & $1.375(12)$ \\
\hline C(39)-H(39) & 0.9500 \\
\hline $\mathrm{C}(40)-\mathrm{H}(40)$ & 0.9500 \\
\hline$C(41)-C(44)$ & $1.508(12)$ \\
\hline $\mathrm{C}(41)-\mathrm{C}(43)$ & $1.511(12)$ \\
\hline $\mathrm{C}(41)-\mathrm{C}(42)$ & $1.540(11)$ \\
\hline $\mathrm{C}(42)-\mathrm{H}(42 \mathrm{~A})$ & 0.9800 \\
\hline
\end{tabular}




\begin{tabular}{|c|c|}
\hline $\mathrm{C}(42)-\mathrm{H}(42 \mathrm{~B})$ & 0.9800 \\
\hline $\mathrm{C}(42)-\mathrm{H}(42 \mathrm{C})$ & 0.9800 \\
\hline $\mathrm{C}(43)-\mathrm{H}(43 \mathrm{~A})$ & 0.9800 \\
\hline $\mathrm{C}(43)-\mathrm{H}(43 \mathrm{~B})$ & 0.9800 \\
\hline $\mathrm{C}(43)-\mathrm{H}(43 \mathrm{C})$ & 0.9800 \\
\hline $\mathrm{C}(44)-\mathrm{H}(44 \mathrm{~A})$ & 0.9800 \\
\hline $\mathrm{C}(44)-\mathrm{H}(44 \mathrm{~B})$ & 0.9800 \\
\hline $\mathrm{C}(44)-\mathrm{H}(44 \mathrm{C})$ & 0.9800 \\
\hline $\mathrm{O}(4)-\mathrm{S}(1)-\mathrm{N}(2)$ & $110.5(3)$ \\
\hline $\mathrm{O}(4)-\mathrm{S}(1)-\mathrm{C}(19)$ & $105.8(3)$ \\
\hline $\mathrm{N}(2)-\mathrm{S}(1)-\mathrm{C}(19)$ & $99.2(3)$ \\
\hline $\mathrm{O}(8)-\mathrm{S}(2)-\mathrm{N}(4)$ & $110.3(3)$ \\
\hline $\mathrm{O}(8)-\mathrm{S}(2)-\mathrm{C}(41)$ & $106.2(4)$ \\
\hline $\mathrm{N}(4)-\mathrm{S}(2)-\mathrm{C}(41)$ & $98.6(3)$ \\
\hline $\mathrm{C}(5)-\mathrm{N}(1)-\mathrm{C}(6)$ & $117.3(6)$ \\
\hline $\mathrm{C}(2)-\mathrm{N}(2)-\mathrm{S}(1)$ & $115.9(4)$ \\
\hline $\mathrm{C}(2)-\mathrm{N}(2)-\mathrm{H}(2 \mathrm{~A})$ & 116(6) \\
\hline $\mathrm{S}(1)-\mathrm{N}(2)-\mathrm{H}(2 \mathrm{~A})$ & $114(6)$ \\
\hline $\mathrm{C}(28)-\mathrm{N}(3)-\mathrm{C}(27)$ & $116.6(6)$ \\
\hline $\mathrm{C}(24)-\mathrm{N}(4)-\mathrm{S}(2)$ & $116.2(4)$ \\
\hline $\mathrm{C}(24)-\mathrm{N}(4)-\mathrm{H}(4)$ & 109(4) \\
\hline $\mathrm{S}(2)-\mathrm{N}(4)-\mathrm{H}(4)$ & $111(4)$ \\
\hline $\mathrm{C}(7)-\mathrm{O}(1)-\mathrm{C}(12)$ & $117.9(5)$ \\
\hline $\mathrm{C}(9)-\mathrm{O}(3)-\mathrm{C}(10)$ & $115.3(5)$ \\
\hline $\mathrm{C}(29)-\mathrm{O}(5)-\mathrm{C}(34)$ & $118.5(6)$ \\
\hline $\mathrm{C}(31)-\mathrm{O}(7)-\mathrm{C}(32)$ & $116.9(6)$ \\
\hline $\mathrm{H}(9 \mathrm{~A})-\mathrm{O}(9)-\mathrm{H}(9 \mathrm{~B})$ & $96(10)$ \\
\hline $\mathrm{C}(8)-\mathrm{C}(1)-\mathrm{C}(5)$ & $120.4(6)$ \\
\hline $\mathrm{C}(8)-\mathrm{C}(1)-\mathrm{C}(2)$ & $128.4(6)$ \\
\hline$C(5)-C(1)-C(2)$ & $111.2(6)$ \\
\hline $\mathrm{N}(2)-\mathrm{C}(2)-\mathrm{C}(1)$ & $107.5(5)$ \\
\hline $\mathrm{N}(2)-\mathrm{C}(2)-\mathrm{C}(3)$ & $115.9(6)$ \\
\hline $\mathrm{C}(1)-\mathrm{C}(2)-\mathrm{C}(3)$ & $102.7(6)$ \\
\hline $\mathrm{N}(2)-\mathrm{C}(2)-\mathrm{H}(2)$ & 110.1 \\
\hline $\mathrm{C}(1)-\mathrm{C}(2)-\mathrm{H}(2)$ & 110.1 \\
\hline $\mathrm{C}(3)-\mathrm{C}(2)-\mathrm{H}(2)$ & 110.1 \\
\hline$C(4)-C(3)-C(2)$ & $106.2(5)$ \\
\hline
\end{tabular}




\begin{tabular}{|c|c|}
\hline $\mathrm{C}(4)-\mathrm{C}(3)-\mathrm{H}(3 \mathrm{~A})$ & 110.5 \\
\hline$C(2)-C(3)-H(3 A)$ & 110.5 \\
\hline $\mathrm{C}(4)-\mathrm{C}(3)-\mathrm{H}(3 \mathrm{~B})$ & 110.5 \\
\hline $\mathrm{C}(2)-\mathrm{C}(3)-\mathrm{H}(3 \mathrm{~B})$ & 110.5 \\
\hline $\mathrm{H}(3 \mathrm{~A})-\mathrm{C}(3)-\mathrm{H}(3 \mathrm{~B})$ & 108.7 \\
\hline$C(5)-C(4)-C(3)$ & $103.1(6)$ \\
\hline $\mathrm{C}(5)-\mathrm{C}(4)-\mathrm{H}(4 \mathrm{C})$ & 111.1 \\
\hline $\mathrm{C}(3)-\mathrm{C}(4)-\mathrm{H}(4 \mathrm{C})$ & 111.1 \\
\hline$C(5)-C(4)-H(4 D)$ & 111.1 \\
\hline $\mathrm{C}(3)-\mathrm{C}(4)-\mathrm{H}(4 \mathrm{D})$ & 111.1 \\
\hline $\mathrm{H}(4 \mathrm{C})-\mathrm{C}(4)-\mathrm{H}(4 \mathrm{D})$ & 109.1 \\
\hline$N(1)-C(5)-C(1)$ & $123.4(6)$ \\
\hline $\mathrm{N}(1)-\mathrm{C}(5)-\mathrm{C}(4)$ & $123.9(6)$ \\
\hline$C(1)-C(5)-C(4)$ & $112.7(6)$ \\
\hline $\mathrm{N}(1)-\mathrm{C}(6)-\mathrm{C}(7)$ & $122.7(6)$ \\
\hline $\mathrm{N}(1)-\mathrm{C}(6)-\mathrm{H}(6)$ & 118.7 \\
\hline $\mathrm{C}(7)-\mathrm{C}(6)-\mathrm{H}(6)$ & 118.7 \\
\hline $\mathrm{O}(1)-\mathrm{C}(7)-\mathrm{C}(6)$ & $124.5(6)$ \\
\hline $\mathrm{O}(1)-\mathrm{C}(7)-\mathrm{C}(8)$ & $116.3(6)$ \\
\hline$C(6)-C(7)-C(8)$ & $119.3(6)$ \\
\hline $\mathrm{C}(1)-\mathrm{C}(8)-\mathrm{C}(7)$ & $117.0(6)$ \\
\hline$C(1)-C(8)-C(9)$ & $120.3(6)$ \\
\hline $\mathrm{C}(7)-\mathrm{C}(8)-\mathrm{C}(9)$ & $122.6(6)$ \\
\hline $\mathrm{O}(2)-\mathrm{C}(9)-\mathrm{O}(3)$ & $124.1(6)$ \\
\hline $\mathrm{O}(2)-\mathrm{C}(9)-\mathrm{C}(8)$ & $122.6(6)$ \\
\hline $\mathrm{O}(3)-\mathrm{C}(9)-\mathrm{C}(8)$ & $113.3(6)$ \\
\hline $\mathrm{O}(3)-\mathrm{C}(10)-\mathrm{C}(11)$ & $108.0(6)$ \\
\hline $\mathrm{O}(3)-\mathrm{C}(10)-\mathrm{H}(10 \mathrm{~A})$ & 110.1 \\
\hline $\mathrm{C}(11)-\mathrm{C}(10)-\mathrm{H}(10 \mathrm{~A})$ & 110.1 \\
\hline $\mathrm{O}(3)-\mathrm{C}(10)-\mathrm{H}(10 \mathrm{~B})$ & 110.1 \\
\hline $\mathrm{C}(11)-\mathrm{C}(10)-\mathrm{H}(10 \mathrm{~B})$ & 110.1 \\
\hline $\mathrm{H}(10 \mathrm{~A})-\mathrm{C}(10)-\mathrm{H}(10 \mathrm{~B})$ & 108.4 \\
\hline$C(10)-C(11)-H(11 A)$ & 109.5 \\
\hline $\mathrm{C}(10)-\mathrm{C}(11)-\mathrm{H}(11 \mathrm{~B})$ & 109.5 \\
\hline $\mathrm{H}(11 \mathrm{~A})-\mathrm{C}(11)-\mathrm{H}(11 \mathrm{~B})$ & 109.5 \\
\hline $\mathrm{C}(10)-\mathrm{C}(11)-\mathrm{H}(11 \mathrm{C})$ & 109.5 \\
\hline $\mathrm{H}(11 \mathrm{~A})-\mathrm{C}(11)-\mathrm{H}(11 \mathrm{C})$ & 109.5 \\
\hline $\mathrm{H}(11 \mathrm{~B})-\mathrm{C}(11)-\mathrm{H}(11 \mathrm{C})$ & 109.5 \\
\hline
\end{tabular}




\begin{tabular}{|c|c|}
\hline $\mathrm{O}(1)-\mathrm{C}(12)-\mathrm{C}(13)$ & $113.7(6)$ \\
\hline $\mathrm{O}(1)-\mathrm{C}(12)-\mathrm{H}(12 \mathrm{~A})$ & 108.8 \\
\hline $\mathrm{C}(13)-\mathrm{C}(12)-\mathrm{H}(12 \mathrm{~A})$ & 108.8 \\
\hline $\mathrm{O}(1)-\mathrm{C}(12)-\mathrm{H}(12 \mathrm{~B})$ & 108.8 \\
\hline $\mathrm{C}(13)-\mathrm{C}(12)-\mathrm{H}(12 \mathrm{~B})$ & 108.8 \\
\hline $\mathrm{H}(12 \mathrm{~A})-\mathrm{C}(12)-\mathrm{H}(12 \mathrm{~B})$ & 107.7 \\
\hline $\mathrm{C}(14)-\mathrm{C}(13)-\mathrm{C}(18)$ & $118.8(7)$ \\
\hline$C(14)-C(13)-C(12)$ & $122.1(7)$ \\
\hline$C(18)-C(13)-C(12)$ & 119.1(6) \\
\hline$C(15)-C(14)-C(13)$ & $120.9(7)$ \\
\hline $\mathrm{C}(15)-\mathrm{C}(14)-\mathrm{H}(14)$ & 119.5 \\
\hline $\mathrm{C}(13)-\mathrm{C}(14)-\mathrm{H}(14)$ & 119.5 \\
\hline $\mathrm{C}(14)-\mathrm{C}(15)-\mathrm{C}(16)$ & $119.4(7)$ \\
\hline $\mathrm{C}(14)-\mathrm{C}(15)-\mathrm{H}(15)$ & 120.3 \\
\hline $\mathrm{C}(16)-\mathrm{C}(15)-\mathrm{H}(15)$ & 120.3 \\
\hline$C(17)-C(16)-C(15)$ & $119.6(7)$ \\
\hline $\mathrm{C}(17)-\mathrm{C}(16)-\mathrm{H}(16)$ & 120.2 \\
\hline$C(15)-C(16)-H(16)$ & 120.2 \\
\hline $\mathrm{C}(18)-\mathrm{C}(17)-\mathrm{C}(16)$ & $121.1(7)$ \\
\hline $\mathrm{C}(18)-\mathrm{C}(17)-\mathrm{H}(17)$ & 119.4 \\
\hline $\mathrm{C}(16)-\mathrm{C}(17)-\mathrm{H}(17)$ & 119.4 \\
\hline$C(17)-C(18)-C(13)$ & $120.1(7)$ \\
\hline $\mathrm{C}(17)-\mathrm{C}(18)-\mathrm{H}(18)$ & 120.0 \\
\hline $\mathrm{C}(13)-\mathrm{C}(18)-\mathrm{H}(18)$ & 120.0 \\
\hline$C(21)-C(19)-C(20)$ & $110.5(7)$ \\
\hline $\mathrm{C}(21)-\mathrm{C}(19)-\mathrm{C}(22)$ & $111.4(8)$ \\
\hline$C(20)-C(19)-C(22)$ & $111.6(7)$ \\
\hline $\mathrm{C}(21)-\mathrm{C}(19)-\mathrm{S}(1)$ & $107.1(6)$ \\
\hline$C(20)-C(19)-S(1)$ & $111.0(5)$ \\
\hline $\mathrm{C}(22)-\mathrm{C}(19)-\mathrm{S}(1)$ & $105.0(6)$ \\
\hline$C(19)-C(20)-H(20 A)$ & 109.5 \\
\hline $\mathrm{C}(19)-\mathrm{C}(20)-\mathrm{H}(20 \mathrm{~B})$ & 109.5 \\
\hline $\mathrm{H}(20 \mathrm{~A})-\mathrm{C}(20)-\mathrm{H}(20 \mathrm{~B})$ & 109.5 \\
\hline $\mathrm{C}(19)-\mathrm{C}(20)-\mathrm{H}(20 \mathrm{C})$ & 109.5 \\
\hline $\mathrm{H}(20 \mathrm{~A})-\mathrm{C}(20)-\mathrm{H}(20 \mathrm{C})$ & 109.5 \\
\hline $\mathrm{H}(20 \mathrm{~B})-\mathrm{C}(20)-\mathrm{H}(20 \mathrm{C})$ & 109.5 \\
\hline $\mathrm{C}(19)-\mathrm{C}(21)-\mathrm{H}(21 \mathrm{~A})$ & 109.5 \\
\hline $\mathrm{C}(19)-\mathrm{C}(21)-\mathrm{H}(21 \mathrm{~B})$ & 109.5 \\
\hline
\end{tabular}




\begin{tabular}{|c|c|}
\hline $\mathrm{H}(21 \mathrm{~A})-\mathrm{C}(21)-\mathrm{H}(21 \mathrm{~B})$ & 109.5 \\
\hline $\mathrm{C}(19)-\mathrm{C}(21)-\mathrm{H}(21 \mathrm{C})$ & 109.5 \\
\hline $\mathrm{H}(21 \mathrm{~A})-\mathrm{C}(21)-\mathrm{H}(21 \mathrm{C})$ & 109.5 \\
\hline $\mathrm{H}(21 \mathrm{~B})-\mathrm{C}(21)-\mathrm{H}(21 \mathrm{C})$ & 109.5 \\
\hline $\mathrm{C}(19)-\mathrm{C}(22)-\mathrm{H}(22 \mathrm{~A})$ & 109.5 \\
\hline $\mathrm{C}(19)-\mathrm{C}(22)-\mathrm{H}(22 \mathrm{~B})$ & 109.5 \\
\hline $\mathrm{H}(22 \mathrm{~A})-\mathrm{C}(22)-\mathrm{H}(22 \mathrm{~B})$ & 109.5 \\
\hline $\mathrm{C}(19)-\mathrm{C}(22)-\mathrm{H}(22 \mathrm{C})$ & 109.5 \\
\hline $\mathrm{H}(22 \mathrm{~A})-\mathrm{C}(22)-\mathrm{H}(22 \mathrm{C})$ & 109.5 \\
\hline $\mathrm{H}(22 \mathrm{~B})-\mathrm{C}(22)-\mathrm{H}(22 \mathrm{C})$ & 109.5 \\
\hline $\mathrm{C}(27)-\mathrm{C}(23)-\mathrm{C}(30)$ & $120.6(6)$ \\
\hline $\mathrm{C}(27)-\mathrm{C}(23)-\mathrm{C}(24)$ & $111.1(6)$ \\
\hline$C(30)-C(23)-C(24)$ & $128.2(6)$ \\
\hline $\mathrm{N}(4)-\mathrm{C}(24)-\mathrm{C}(23)$ & $107.9(5)$ \\
\hline $\mathrm{N}(4)-\mathrm{C}(24)-\mathrm{C}(25)$ & $116.9(5)$ \\
\hline $\mathrm{C}(23)-\mathrm{C}(24)-\mathrm{C}(25)$ & $102.3(5)$ \\
\hline $\mathrm{N}(4)-\mathrm{C}(24)-\mathrm{H}(24)$ & 109.8 \\
\hline $\mathrm{C}(23)-\mathrm{C}(24)-\mathrm{H}(24)$ & 109.8 \\
\hline $\mathrm{C}(25)-\mathrm{C}(24)-\mathrm{H}(24)$ & 109.8 \\
\hline $\mathrm{C}(26)-\mathrm{C}(25)-\mathrm{C}(24)$ & $108.2(5)$ \\
\hline$C(26)-C(25)-H(25 A)$ & 110.1 \\
\hline $\mathrm{C}(24)-\mathrm{C}(25)-\mathrm{H}(25 \mathrm{~A})$ & 110.1 \\
\hline $\mathrm{C}(26)-\mathrm{C}(25)-\mathrm{H}(25 \mathrm{~B})$ & 110.1 \\
\hline $\mathrm{C}(24)-\mathrm{C}(25)-\mathrm{H}(25 \mathrm{~B})$ & 110.1 \\
\hline $\mathrm{H}(25 \mathrm{~A})-\mathrm{C}(25)-\mathrm{H}(25 \mathrm{~B})$ & 108.4 \\
\hline $\mathrm{C}(27)-\mathrm{C}(26)-\mathrm{C}(25)$ & $102.7(6)$ \\
\hline $\mathrm{C}(27)-\mathrm{C}(26)-\mathrm{H}(26 \mathrm{~A})$ & 111.2 \\
\hline $\mathrm{C}(25)-\mathrm{C}(26)-\mathrm{H}(26 \mathrm{~A})$ & 111.2 \\
\hline $\mathrm{C}(27)-\mathrm{C}(26)-\mathrm{H}(26 \mathrm{~B})$ & 111.2 \\
\hline $\mathrm{C}(25)-\mathrm{C}(26)-\mathrm{H}(26 \mathrm{~B})$ & 111.2 \\
\hline $\mathrm{H}(26 \mathrm{~A})-\mathrm{C}(26)-\mathrm{H}(26 \mathrm{~B})$ & 109.1 \\
\hline $\mathrm{N}(3)-\mathrm{C}(27)-\mathrm{C}(23)$ & $123.2(6)$ \\
\hline $\mathrm{N}(3)-\mathrm{C}(27)-\mathrm{C}(26)$ & $123.5(6)$ \\
\hline$C(23)-C(27)-C(26)$ & 113.3(6) \\
\hline $\mathrm{N}(3)-\mathrm{C}(28)-\mathrm{C}(29)$ & $124.1(6)$ \\
\hline $\mathrm{N}(3)-\mathrm{C}(28)-\mathrm{H}(28)$ & 117.9 \\
\hline $\mathrm{C}(29)-\mathrm{C}(28)-\mathrm{H}(28)$ & 117.9 \\
\hline $\mathrm{O}(5)-\mathrm{C}(29)-\mathrm{C}(28)$ & $125.0(6)$ \\
\hline
\end{tabular}




$\begin{array}{ll}\mathrm{O}(5)-\mathrm{C}(29)-\mathrm{C}(30) & 116.8(6) \\ \mathrm{C}(28)-\mathrm{C}(29)-\mathrm{C}(30) & 118.1(6) \\ \mathrm{C}(23)-\mathrm{C}(30)-\mathrm{C}(29) & 117.3(6) \\ \mathrm{C}(23)-\mathrm{C}(30)-\mathrm{C}(31) & 123.4(6) \\ \mathrm{C}(29)-\mathrm{C}(30)-\mathrm{C}(31) & 119.2(6) \\ \mathrm{O}(6)-\mathrm{C}(31)-\mathrm{O}(7) & 123.4(7) \\ \mathrm{O}(6)-\mathrm{C}(31)-\mathrm{C}(30) & 127.6(6) \\ \mathrm{O}(7)-\mathrm{C}(31)-\mathrm{C}(30) & 109.0(6) \\ \mathrm{O}(7)-\mathrm{C}(32)-\mathrm{C}(33) & 108.4(7) \\ \mathrm{O}(7)-\mathrm{C}(32)-\mathrm{H}(32 \mathrm{~A}) & 110.0 \\ \mathrm{C}(33)-\mathrm{C}(32)-\mathrm{H}(32 \mathrm{~A}) & 110.0 \\ \mathrm{O}(7)-\mathrm{C}(32)-\mathrm{H}(32 \mathrm{~B}) & 110.0 \\ \mathrm{C}(33)-\mathrm{C}(32)-\mathrm{H}(32 \mathrm{~B}) & 110.0 \\ \mathrm{H}(32 \mathrm{~A})-\mathrm{C}(32)-\mathrm{H}(32 \mathrm{~B}) & 108.4 \\ \mathrm{C}(32)-\mathrm{C}(33)-\mathrm{H}(33 \mathrm{~A}) & 109.5 \\ \mathrm{C}(32)-\mathrm{C}(33)-\mathrm{H}(33 \mathrm{~B}) & 109.5 \\ \mathrm{H}(33 \mathrm{~A})-\mathrm{C}(33)-\mathrm{H}(33 \mathrm{~B}) & 109.5 \\ \mathrm{C}(32)-\mathrm{C}(33)-\mathrm{H}(33 \mathrm{C}) & 109.5 \\ \mathrm{H}(33 \mathrm{~A})-\mathrm{C}(33)-\mathrm{H}(33 \mathrm{C}) & 109.5 \\ \mathrm{H}(33 \mathrm{~B})-\mathrm{C}(33)-\mathrm{H}(33 \mathrm{C}) & 109.5 \\ \mathrm{O}(5)-\mathrm{C}(34)-\mathrm{C}(35) & 113.2(5) \\ \mathrm{O}(5)-\mathrm{C}(34)-\mathrm{H}(34 \mathrm{~A}) & 108.9 \\ \mathrm{C}(35)-\mathrm{C}(34)-\mathrm{H}(34 \mathrm{~A}) & 108.9 \\ \mathrm{O}(5)-\mathrm{C}(34)-\mathrm{H}(34 \mathrm{~B}) & 108.9 \\ \mathrm{C}(35)-\mathrm{C}(34)-\mathrm{H}(34 \mathrm{~B}) & 108.9 \\ \mathrm{H}(34 \mathrm{~A})-\mathrm{C}(34)-\mathrm{H}(34 \mathrm{~B}) & 107.7 \\ \mathrm{C}(40)-\mathrm{C}(35)-\mathrm{C}(36) & 118.2(7) \\ \mathrm{C}(40)-\mathrm{C}(35)-\mathrm{C}(34) & 121.7(7) \\ \mathrm{C}(36)-\mathrm{C}(35)-\mathrm{C}(34) & 120.1(6) \\ \mathrm{C}(37)-\mathrm{C}(36)-\mathrm{C}(35) & 120.9(7) \\ \mathrm{C}(37)-\mathrm{C}(36)-\mathrm{H}(36) & 119.5 \\ \mathrm{C}(35)-\mathrm{C}(36)-\mathrm{H}(36) & 119.5 \\ \mathrm{C}(36)-\mathrm{C}(37)-\mathrm{C}(38) & 120.8(8) \\ \mathrm{C}(36)-\mathrm{C}(37)-\mathrm{H}(37) & 119.6 \\ \mathrm{C}(38) & 119.6 \\ \mathrm{C}(38) & 120.3 \\ \end{array}$




$\begin{array}{ll}\mathrm{C}(40)-\mathrm{C}(39)-\mathrm{C}(38) & 119.9(8) \\ \mathrm{C}(40)-\mathrm{C}(39)-\mathrm{H}(39) & 120.0 \\ \mathrm{C}(38)-\mathrm{C}(39)-\mathrm{H}(39) & 120.0 \\ \mathrm{C}(39)-\mathrm{C}(40)-\mathrm{C}(35) & 120.8(8) \\ \mathrm{C}(39)-\mathrm{C}(40)-\mathrm{H}(40) & 119.6 \\ \mathrm{C}(35)-\mathrm{C}(40)-\mathrm{H}(40) & 119.6 \\ \mathrm{C}(44)-\mathrm{C}(41)-\mathrm{C}(43) & 113.2(7) \\ \mathrm{C}(44)-\mathrm{C}(41)-\mathrm{C}(42) & 111.0(7) \\ \mathrm{C}(43)-\mathrm{C}(41)-\mathrm{C}(42) & 111.7(8) \\ \mathrm{C}(44)-\mathrm{C}(41)-\mathrm{S}(2) & 109.9(6) \\ \mathrm{C}(43)-\mathrm{C}(41)-\mathrm{S}(2) & 106.3(5) \\ \mathrm{C}(42)-\mathrm{C}(41)-\mathrm{S}(2) & 104.1(5) \\ \mathrm{C}(41)-\mathrm{C}(42)-\mathrm{H}(42 \mathrm{~A}) & 109.5 \\ \mathrm{C}(41)-\mathrm{C}(42)-\mathrm{H}(42 \mathrm{~B}) & 109.5 \\ \mathrm{H}(42 \mathrm{~A})-\mathrm{C}(42)-\mathrm{H}(42 \mathrm{~B}) & 109.5 \\ \mathrm{C}(41)-\mathrm{C}(42)-\mathrm{H}(42 \mathrm{C}) & 109.5 \\ \mathrm{H}(42 \mathrm{~A})-\mathrm{C}(42)-\mathrm{H}(42 \mathrm{C}) & 109.5 \\ \mathrm{H}(42 \mathrm{~B})-\mathrm{C}(42)-\mathrm{H}(42 \mathrm{C}) & 109.5 \\ \mathrm{C}(41)-\mathrm{C}(43)-\mathrm{H}(43 \mathrm{~A}) & 109.5 \\ \mathrm{C}(41)-\mathrm{C}(43)-\mathrm{H}(43 \mathrm{~B}) & 109.5 \\ \mathrm{H}(43 \mathrm{~A})-\mathrm{C}(43)-\mathrm{H}(43 \mathrm{~B}) & 109.5 \\ \mathrm{C}(41)-\mathrm{C}(43)-\mathrm{H}(43 \mathrm{C}) & 109.5 \\ \mathrm{H}(43 \mathrm{~A})-\mathrm{C}(43)-\mathrm{H}(43 \mathrm{C}) & 109.5 \\ \mathrm{H}(43 \mathrm{~B})-\mathrm{C}(43)-\mathrm{H}(43 \mathrm{C}) & 109.5 \\ \mathrm{C}(41)-\mathrm{C}(44)-\mathrm{H}(44 \mathrm{~A}) & 109.5 \\ \mathrm{C}(41)-\mathrm{C}(44)-\mathrm{H}(44 \mathrm{~B}) & 109.5 \\ \mathrm{H}(44 \mathrm{~A})-\mathrm{C}(44)-\mathrm{H}(44 \mathrm{~B}) & 109.5 \\ \mathrm{C}(41)-\mathrm{C}(44)-\mathrm{H}(44 \mathrm{C}) & 109.5 \\ \mathrm{H}(44 \mathrm{~A})-\mathrm{C}(44)-\mathrm{H}(44 \mathrm{C}) & 109.5 \\ \mathrm{H}(44 \mathrm{~B})-\mathrm{C}(44)-\mathrm{H}(44 \mathrm{C}) & 109.5 \\ & \end{array}$

Symmetry transformations used to generate equivalent atoms: 
Table 4. Anisotropic displacement parameters $\quad\left(\AA^{2} \times 10^{3}\right)$ for mo_(S,R)-8_0m.

The anisotropic displacement factor exponent takes the form: $\quad-2 p^{2}\left[h^{2} a^{* 2} U^{11}+\ldots\right.$ $+2 \mathrm{~h} \mathrm{k} \mathrm{a}^{*} \mathrm{~b}^{*} \mathrm{U}^{12}$ ]

\begin{tabular}{|c|c|c|c|c|c|c|}
\hline & $\mathrm{U}^{11}$ & $\mathrm{U}^{22}$ & $\mathrm{U}^{33}$ & $\mathrm{U}^{23}$ & $\mathrm{U}^{13}$ & $\mathrm{U}^{12}$ \\
\hline$S(1)$ & $27(1)$ & $27(1)$ & $23(1)$ & $-2(1)$ & $7(1)$ & $-1(1)$ \\
\hline$S(2)$ & $26(1)$ & $41(1)$ & $21(1)$ & $4(1)$ & $7(1)$ & $-1(1)$ \\
\hline $\mathrm{N}(1)$ & $23(3)$ & $26(3)$ & $31(3)$ & $1(2)$ & $6(2)$ & $0(2)$ \\
\hline $\mathrm{N}(2)$ & $30(3)$ & $15(3)$ & $20(3)$ & $-4(2)$ & $7(2)$ & $0(2)$ \\
\hline $\mathrm{N}(3)$ & $34(3)$ & $19(3)$ & $29(3)$ & $4(2)$ & $14(3)$ & $2(2)$ \\
\hline $\mathrm{N}(4)$ & $25(3)$ & $19(3)$ & $22(3)$ & $5(2)$ & $6(2)$ & $3(2)$ \\
\hline $\mathrm{O}(1)$ & $32(3)$ & $28(3)$ & $25(2)$ & $7(2)$ & $3(2)$ & $-5(2)$ \\
\hline $\mathrm{O}(2)$ & $71(4)$ & $23(3)$ & $50(3)$ & $-9(2)$ & $37(3)$ & $-9(3)$ \\
\hline $\mathrm{O}(3)$ & $37(3)$ & $21(2)$ & $32(3)$ & $-1(2)$ & $16(2)$ & $-7(2)$ \\
\hline $\mathrm{O}(4)$ & $36(3)$ & $33(3)$ & $24(2)$ & $3(2)$ & $2(2)$ & $1(2)$ \\
\hline $\mathrm{O}(5)$ & $40(3)$ & $35(3)$ & $24(2)$ & $-4(2)$ & $13(2)$ & $-5(2)$ \\
\hline $\mathrm{O}(6)$ & $33(3)$ & $33(3)$ & $40(3)$ & $0(2)$ & $0(2)$ & $-9(2)$ \\
\hline $\mathrm{O}(7)$ & $37(3)$ & $21(2)$ & $41(3)$ & $1(2)$ & $2(2)$ & $-1(2)$ \\
\hline $\mathrm{O}(8)$ & $41(3)$ & $68(4)$ & $28(3)$ & $-16(3)$ & $12(2)$ & $-2(3)$ \\
\hline $\mathrm{O}(9)$ & $38(4)$ & $67(5)$ & $69(5)$ & $-20(4)$ & $-2(3)$ & $6(4)$ \\
\hline $\mathrm{C}(1)$ & $21(3)$ & $27(3)$ & $29(3)$ & $-2(3)$ & $12(3)$ & $0(3)$ \\
\hline$C(2)$ & $31(4)$ & $20(3)$ & $23(3)$ & $-2(3)$ & $8(3)$ & $4(3)$ \\
\hline$C(3)$ & $32(4)$ & $35(4)$ & $28(4)$ & $6(3)$ & $10(3)$ & $-3(3)$ \\
\hline$C(4)$ & $32(4)$ & $26(4)$ & $31(4)$ & $8(3)$ & $6(3)$ & $-3(3)$ \\
\hline$C(5)$ & $24(3)$ & $17(3)$ & $32(4)$ & $3(3)$ & $8(3)$ & $1(3)$ \\
\hline$C(6)$ & $28(3)$ & $24(3)$ & $21(3)$ & $-2(3)$ & 1(3) & $-3(3)$ \\
\hline$C(7)$ & $25(3)$ & $26(4)$ & $25(3)$ & $2(3)$ & $8(3)$ & 1(3) \\
\hline$C(8)$ & $23(3)$ & $19(3)$ & $24(3)$ & $1(3)$ & $8(2)$ & $0(3)$ \\
\hline C(9) & $30(4)$ & $24(3)$ & $29(4)$ & 1(3) & $12(3)$ & $0(3)$ \\
\hline$C(10)$ & $46(5)$ & $18(3)$ & $40(4)$ & $1(3)$ & $15(4)$ & $-2(3)$ \\
\hline $\mathrm{C}(11)$ & $58(5)$ & $27(4)$ & $40(4)$ & $-3(3)$ & $23(4)$ & $-10(4)$ \\
\hline$C(12)$ & $31(4)$ & $30(4)$ & $26(3)$ & $1(3)$ & $4(3)$ & $1(3)$ \\
\hline $\mathrm{C}(13)$ & $37(4)$ & $24(4)$ & $27(3)$ & $7(3)$ & $14(3)$ & $6(3)$ \\
\hline$C(14)$ & $44(4)$ & $21(3)$ & $33(4)$ & $1(3)$ & $14(3)$ & $0(3)$ \\
\hline
\end{tabular}




$\begin{array}{llllccc}\mathrm{C}(15) & 50(5) & 27(4) & 44(4) & 7(3) & 24(4) & 11(3) \\ \mathrm{C}(16) & 30(4) & 49(5) & 42(4) & 21(4) & 16(3) & 9(4) \\ \mathrm{C}(17) & 35(4) & 33(4) & 33(4) & 10(3) & 1(3) & 2(3) \\ \mathrm{C}(18) & 36(4) & 23(3) & 22(3) & 5(3) & 7(3) & 7(3) \\ \mathrm{C}(19) & 32(4) & 35(4) & 35(4) & 1(3) & 9(3) & -8(3) \\ \mathrm{C}(20) & 37(4) & 44(4) & 58(5) & -2(4) & 28(4) & -5(4) \\ \mathrm{C}(21) & 49(5) & 34(4) & 72(6) & 11(4) & 20(5) & -8(4) \\ \mathrm{C}(22) & 54(6) & 57(6) & 47(5) & -1(4) & 9(4) & -27(5) \\ \mathrm{C}(23) & 22(3) & 16(3) & 26(3) & 1(2) & 10(3) & 0(2) \\ \mathrm{C}(24) & 28(3) & 21(3) & 17(3) & 2(2) & 4(3) & 4(3) \\ \mathrm{C}(25) & 34(4) & 27(4) & 28(4) & 3(3) & 10(3) & -2(3) \\ \mathrm{C}(26) & 40(4) & 25(3) & 23(3) & 0(3) & 12(3) & -6(3) \\ \mathrm{C}(27) & 24(3) & 26(3) & 24(3) & -2(3) & 10(3) & 2(3) \\ \mathrm{C}(28) & 34(4) & 30(4) & 22(3) & 4(3) & 9(3) & 1(3) \\ \mathrm{C}(29) & 26(3) & 23(3) & 22(3) & -4(3) & 6(3) & -4(3) \\ \mathrm{C}(30) & 22(3) & 27(3) & 21(3) & -2(3) & 6(2) & -3(3) \\ \mathrm{C}(31) & 25(4) & 27(3) & 19(3) & -6(3) & 4(3) & -2(3) \\ \mathrm{C}(32) & 46(5) & 23(4) & 42(4) & -5(3) & 5(4) & -3(3) \\ \mathrm{C}(33) & 46(5) & 27(4) & 59(5) & -11(4) & 9(4) & 3(4) \\ \mathrm{C}(34) & 31(4) & 44(4) & 13(3) & -5(3) & 5(3) & -1(3) \\ \mathrm{C}(35) & 29(3) & 33(4) & 22(3) & -8(3) & 5(3) & 2(3) \\ \mathrm{C}(36) & 38(4) & 28(3) & 21(3) & -9(3) & 7(3) & 1(3) \\ \mathrm{C}(37) & 40(4) & 42(4) & 26(4) & -10(3) & 11(3) & -7(4) \\ \mathrm{C}(38) & 34(4) & 37(4) & 52(5) & -17(4) & 18(4) & -7(3) \\ \mathrm{C}(39) & 43(5) & 45(5) & 35(4) & -18(4) & -3(4) & 12(4) \\ \mathrm{C}(40) & 37(4) & 34(4) & 28(4) & -10(3) & 2(3) & 0(3) \\ \mathrm{C}(41) & 27(4) & 47(4) & 37(4) & 5(3) & 11(3) & -8(3) \\ \mathrm{C}(42) & 40(5) & 77(7) & 43(5) & 6(5) & 19(4) & -13(5) \\ \mathrm{C}(43) & 43(5) & 36(5) & 71(6) & -8(4) & 18(5) & -16(4) \\ \mathrm{C}(44) & 21(4) & 54(5) & 55(5) & 9(4) & 1(3) & -4(4)\end{array}$


Table 5. Hydrogen coordinates ( x 104) and isotropic displacement parameters $\left(\AA^{2} \mathrm{x} 10^{3}\right)$ for mo_(S,R)-8_0m.

\begin{tabular}{|c|c|c|c|c|}
\hline - & $\mathrm{x}$ & $\mathrm{y}$ & $\mathrm{z}$ & $\mathrm{U}(\mathrm{eq})$ \\
\hline $\mathrm{H}(2)$ & 8063 & 5200 & 9814 & 30 \\
\hline $\mathrm{H}(3 \mathrm{~A})$ & 9250 & 4187 & 10519 & 38 \\
\hline $\mathrm{H}(3 \mathrm{~B})$ & 7317 & 4385 & 10441 & 38 \\
\hline $\mathrm{H}(4 \mathrm{C})$ & 8221 & 3676 & 8829 & 37 \\
\hline $\mathrm{H}(4 \mathrm{D})$ & 6355 & 3716 & 9046 & 37 \\
\hline $\mathrm{H}(6)$ & 4509 & 4418 & 5179 & 31 \\
\hline $\mathrm{H}(10 \mathrm{~A})$ & 9103 & 6675 & 8109 & 41 \\
\hline $\mathrm{H}(10 \mathrm{~B})$ & 7456 & 6853 & 6992 & 41 \\
\hline $\mathrm{H}(11 \mathrm{~A})$ & 10839 & 6587 & 6894 & 60 \\
\hline $\mathrm{H}(11 \mathrm{~B})$ & 9979 & 7184 & 6691 & 60 \\
\hline $\mathrm{H}(11 \mathrm{C})$ & 9196 & 6710 & 5738 & 60 \\
\hline $\mathrm{H}(12 \mathrm{~A})$ & 4333 & 5006 & 3884 & 36 \\
\hline $\mathrm{H}(12 \mathrm{~B})$ & 3876 & 5640 & 3634 & 36 \\
\hline $\mathrm{H}(14)$ & 2224 & 5755 & 5605 & 39 \\
\hline $\mathrm{H}(15)$ & -638 & 5621 & 5579 & 46 \\
\hline $\mathrm{H}(16)$ & -2451 & 4997 & 4220 & 47 \\
\hline $\mathrm{H}(17)$ & -1389 & 4539 & 2889 & 43 \\
\hline $\mathrm{H}(18)$ & 1456 & 4661 & 2922 & 33 \\
\hline $\mathrm{H}(20 \mathrm{~A})$ & 12750 & 5127 & 8450 & 65 \\
\hline $\mathrm{H}(20 \mathrm{~B})$ & 14089 & 4790 & 9512 & 65 \\
\hline $\mathrm{H}(20 \mathrm{C})$ & 14737 & 5326 & 9005 & 65 \\
\hline$H(21 \mathrm{~A})$ & 13196 & 6238 & 9237 & 78 \\
\hline $\mathrm{H}(21 \mathrm{~B})$ & 12001 & 6230 & 10078 & 78 \\
\hline $\mathrm{H}(21 \mathrm{C})$ & 11350 & 5928 & 8818 & 78 \\
\hline $\mathrm{H}(22 \mathrm{~A})$ & 15345 & 5277 & 11516 & 82 \\
\hline $\mathrm{H}(22 \mathrm{~B})$ & 14432 & 5831 & 11732 & 82 \\
\hline $\mathrm{H}(22 \mathrm{C})$ & 15673 & 5837 & 10922 & 82 \\
\hline $\mathrm{H}(24)$ & 8781 & 7835 & 4347 & 27 \\
\hline $\mathrm{H}(25 \mathrm{~A})$ & 8328 & 8834 & 5152 & 36 \\
\hline $\mathrm{H}(25 \mathrm{~B})$ & 10179 & 8607 & 5121 & 36 \\
\hline $\mathrm{H}(26 \mathrm{~A})$ & 8060 & 9403 & 3612 & 35 \\
\hline \multirow[t]{2}{*}{$\mathrm{H}(26 \mathrm{~B})$} & 10107 & 9287 & 3831 & 35 \\
\hline & & S-39 & & \\
\hline
\end{tabular}




\begin{tabular}{|c|c|c|c|c|}
\hline $\mathrm{H}(28)$ & 8018 & 8748 & -157 & 34 \\
\hline $\mathrm{H}(32 \mathrm{~A})$ & 7414 & 6349 & 2771 & 48 \\
\hline $\mathrm{H}(32 \mathrm{~B})$ & 9384 & 6366 & 3673 & 48 \\
\hline $\mathrm{H}(33 \mathrm{~A})$ & 8428 & 6272 & 1141 & 69 \\
\hline $\mathrm{H}(33 \mathrm{~B})$ & 9381 & 5807 & 2074 & 69 \\
\hline $\mathrm{H}(33 \mathrm{C})$ & 10409 & 6356 & 1978 & 69 \\
\hline $\mathrm{H}(34 \mathrm{~A})$ & 6780 & 8233 & -1565 & 36 \\
\hline $\mathrm{H}(34 \mathrm{~B})$ & 6729 & 7603 & -1939 & 36 \\
\hline $\mathrm{H}(36)$ & 8847 & 8476 & -2588 & 35 \\
\hline $\mathrm{H}(37)$ & 11670 & 8489 & -2618 & 43 \\
\hline $\mathrm{H}(38)$ & 13832 & 7967 & -1273 & 48 \\
\hline $\mathrm{H}(39)$ & 13073 & 7385 & 57 & 54 \\
\hline $\mathrm{H}(40)$ & 10214 & 7367 & 84 & 42 \\
\hline $\mathrm{H}(42 \mathrm{~A})$ & 3124 & 7716 & 6036 & 78 \\
\hline $\mathrm{H}(42 \mathrm{~B})$ & 4120 & 7141 & 6180 & 78 \\
\hline $\mathrm{H}(42 \mathrm{C})$ & 2113 & 7186 & 5365 & 78 \\
\hline $\mathrm{H}(43 \mathrm{~A})$ & 3089 & 6776 & 3690 & 76 \\
\hline $\mathrm{H}(43 \mathrm{~B})$ & 5102 & 6789 & 4502 & 76 \\
\hline $\mathrm{H}(43 \mathrm{C})$ & 4489 & 7100 & 3252 & 76 \\
\hline $\mathrm{H}(44 \mathrm{~A})$ & 2870 & 7946 & 2995 & 69 \\
\hline $\mathrm{H}(44 \mathrm{~B})$ & 2579 & 8253 & 4105 & 69 \\
\hline $\mathrm{H}(44 \mathrm{C})$ & 1361 & 7746 & 3500 & 69 \\
\hline $\mathrm{H}(2 \mathrm{~A})$ & $10540(100)$ & $4750(40)$ & $8970(70)$ & $30(20)$ \\
\hline $\mathrm{H}(4)$ & $5640(80)$ & $8370(30)$ & $3700(50)$ & $4(14)$ \\
\hline $\mathrm{H}(9 \mathrm{~A})$ & $5030(170)$ & $8910(50)$ & $6880(130)$ & $110(60)$ \\
\hline $\mathrm{H}(9 \mathrm{~B})$ & $5270(90)$ & $9260(40)$ & $7640(60)$ & $50(30)$ \\
\hline
\end{tabular}


Table 6. Torsion angles $\left[{ }^{\circ}\right]$ for mo_ $(S, R)-8 \_0 \mathrm{~m}$.

\begin{tabular}{|c|c|}
\hline $\mathrm{O}(4)-\mathrm{S}(1)-\mathrm{N}(2)-\mathrm{C}(2)$ & $93.6(5)$ \\
\hline $\mathrm{C}(19)-\mathrm{S}(1)-\mathrm{N}(2)-\mathrm{C}(2)$ & $-155.6(5)$ \\
\hline $\mathrm{O}(8)-\mathrm{S}(2)-\mathrm{N}(4)-\mathrm{C}(24)$ & $93.7(5)$ \\
\hline $\mathrm{C}(41)-\mathrm{S}(2)-\mathrm{N}(4)-\mathrm{C}(24)$ & $-155.3(5)$ \\
\hline $\mathrm{S}(1)-\mathrm{N}(2)-\mathrm{C}(2)-\mathrm{C}(1)$ & $170.9(4)$ \\
\hline $\mathrm{S}(1)-\mathrm{N}(2)-\mathrm{C}(2)-\mathrm{C}(3)$ & $-74.9(6)$ \\
\hline $\mathrm{C}(8)-\mathrm{C}(1)-\mathrm{C}(2)-\mathrm{N}(2)$ & $-69.1(8)$ \\
\hline $\mathrm{C}(5)-\mathrm{C}(1)-\mathrm{C}(2)-\mathrm{N}(2)$ & $108.0(6)$ \\
\hline $\mathrm{C}(8)-\mathrm{C}(1)-\mathrm{C}(2)-\mathrm{C}(3)$ & $168.2(7)$ \\
\hline $\mathrm{C}(5)-\mathrm{C}(1)-\mathrm{C}(2)-\mathrm{C}(3)$ & $-14.8(7)$ \\
\hline $\mathrm{N}(2)-\mathrm{C}(2)-\mathrm{C}(3)-\mathrm{C}(4)$ & $-96.9(7)$ \\
\hline$C(1)-C(2)-C(3)-C(4)$ & $20.0(7)$ \\
\hline $\mathrm{C}(2)-\mathrm{C}(3)-\mathrm{C}(4)-\mathrm{C}(5)$ & $-18.3(8)$ \\
\hline $\mathrm{C}(6)-\mathrm{N}(1)-\mathrm{C}(5)-\mathrm{C}(1)$ & $-0.6(10)$ \\
\hline $\mathrm{C}(6)-\mathrm{N}(1)-\mathrm{C}(5)-\mathrm{C}(4)$ & $-179.7(7)$ \\
\hline $\mathrm{C}(8)-\mathrm{C}(1)-\mathrm{C}(5)-\mathrm{N}(1)$ & $1.5(10)$ \\
\hline $\mathrm{C}(2)-\mathrm{C}(1)-\mathrm{C}(5)-\mathrm{N}(1)$ & $-175.8(6)$ \\
\hline $\mathrm{C}(8)-\mathrm{C}(1)-\mathrm{C}(5)-\mathrm{C}(4)$ & $-179.3(6)$ \\
\hline $\mathrm{C}(2)-\mathrm{C}(1)-\mathrm{C}(5)-\mathrm{C}(4)$ & $3.4(8)$ \\
\hline $\mathrm{C}(3)-\mathrm{C}(4)-\mathrm{C}(5)-\mathrm{N}(1)$ & $-171.1(6)$ \\
\hline $\mathrm{C}(3)-\mathrm{C}(4)-\mathrm{C}(5)-\mathrm{C}(1)$ & $9.7(8)$ \\
\hline $\mathrm{C}(5)-\mathrm{N}(1)-\mathrm{C}(6)-\mathrm{C}(7)$ & $-0.5(10)$ \\
\hline $\mathrm{C}(12)-\mathrm{O}(1)-\mathrm{C}(7)-\mathrm{C}(6)$ & $7.1(10)$ \\
\hline $\mathrm{C}(12)-\mathrm{O}(1)-\mathrm{C}(7)-\mathrm{C}(8)$ & $-171.6(6)$ \\
\hline $\mathrm{N}(1)-\mathrm{C}(6)-\mathrm{C}(7)-\mathrm{O}(1)$ & $-178.1(6)$ \\
\hline $\mathrm{N}(1)-\mathrm{C}(6)-\mathrm{C}(7)-\mathrm{C}(8)$ & $0.5(10)$ \\
\hline $\mathrm{C}(5)-\mathrm{C}(1)-\mathrm{C}(8)-\mathrm{C}(7)$ & $-1.4(9)$ \\
\hline $\mathrm{C}(2)-\mathrm{C}(1)-\mathrm{C}(8)-\mathrm{C}(7)$ & $175.4(6)$ \\
\hline $\mathrm{C}(5)-\mathrm{C}(1)-\mathrm{C}(8)-\mathrm{C}(9)$ & $174.1(6)$ \\
\hline $\mathrm{C}(2)-\mathrm{C}(1)-\mathrm{C}(8)-\mathrm{C}(9)$ & $-9.1(10)$ \\
\hline $\mathrm{O}(1)-\mathrm{C}(7)-\mathrm{C}(8)-\mathrm{C}(1)$ & 179.2(6) \\
\hline$C(6)-C(7)-C(8)-C(1)$ & $0.4(9)$ \\
\hline $\mathrm{O}(1)-\mathrm{C}(7)-\mathrm{C}(8)-\mathrm{C}(9)$ & $3.8(9)$ \\
\hline$C(6)-C(7)-C(8)-C(9)$ & $-175.0(6)$ \\
\hline $\mathrm{C}(10)-\mathrm{O}(3)-\mathrm{C}(9)-\mathrm{O}(2)$ & $-1.9(10)$ \\
\hline $\mathrm{C}(10)-\mathrm{O}(3)-\mathrm{C}(9)-\mathrm{C}(8)$ & 179.1(6) \\
\hline
\end{tabular}




\begin{tabular}{|c|c|}
\hline $\mathrm{C}(1)-\mathrm{C}(8)-\mathrm{C}(9)-\mathrm{O}(2)$ & $-55.5(10)$ \\
\hline $\mathrm{C}(7)-\mathrm{C}(8)-\mathrm{C}(9)-\mathrm{O}(2)$ & $119.7(8)$ \\
\hline $\mathrm{C}(1)-\mathrm{C}(8)-\mathrm{C}(9)-\mathrm{O}(3)$ & $123.5(7)$ \\
\hline $\mathrm{C}(7)-\mathrm{C}(8)-\mathrm{C}(9)-\mathrm{O}(3)$ & $-61.3(9)$ \\
\hline $\mathrm{C}(9)-\mathrm{O}(3)-\mathrm{C}(10)-\mathrm{C}(11)$ & $172.2(6)$ \\
\hline $\mathrm{C}(7)-\mathrm{O}(1)-\mathrm{C}(12)-\mathrm{C}(13)$ & $70.0(8)$ \\
\hline $\mathrm{O}(1)-\mathrm{C}(12)-\mathrm{C}(13)-\mathrm{C}(14)$ & $32.4(9)$ \\
\hline $\mathrm{O}(1)-\mathrm{C}(12)-\mathrm{C}(13)-\mathrm{C}(18)$ & $-149.2(6)$ \\
\hline$C(18)-C(13)-C(14)-C(15)$ & $-1.7(10)$ \\
\hline$C(12)-C(13)-C(14)-C(15)$ & $176.7(7)$ \\
\hline$C(13)-C(14)-C(15)-C(16)$ & $1.0(11)$ \\
\hline$C(14)-C(15)-C(16)-C(17)$ & $-0.6(11)$ \\
\hline$C(15)-C(16)-C(17)-C(18)$ & $1.0(11)$ \\
\hline$C(16)-C(17)-C(18)-C(13)$ & $-1.7(10)$ \\
\hline$C(14)-C(13)-C(18)-C(17)$ & $2.1(10)$ \\
\hline$C(12)-C(13)-C(18)-C(17)$ & $-176.4(6)$ \\
\hline $\mathrm{O}(4)-\mathrm{S}(1)-\mathrm{C}(19)-\mathrm{C}(21)$ & $-177.9(6)$ \\
\hline $\mathrm{N}(2)-\mathrm{S}(1)-\mathrm{C}(19)-\mathrm{C}(21)$ & $67.6(6)$ \\
\hline $\mathrm{O}(4)-\mathrm{S}(1)-\mathrm{C}(19)-\mathrm{C}(20)$ & $61.5(6)$ \\
\hline$N(2)-S(1)-C(19)-C(20)$ & $-53.0(6)$ \\
\hline $\mathrm{O}(4)-\mathrm{S}(1)-\mathrm{C}(19)-\mathrm{C}(22)$ & $-59.3(7)$ \\
\hline $\mathrm{N}(2)-\mathrm{S}(1)-\mathrm{C}(19)-\mathrm{C}(22)$ & $-173.8(6)$ \\
\hline $\mathrm{S}(2)-\mathrm{N}(4)-\mathrm{C}(24)-\mathrm{C}(23)$ & $169.0(4)$ \\
\hline$S(2)-N(4)-C(24)-C(25)$ & $-76.5(6)$ \\
\hline $\mathrm{C}(27)-\mathrm{C}(23)-\mathrm{C}(24)-\mathrm{N}(4)$ & $111.5(6)$ \\
\hline $\mathrm{C}(30)-\mathrm{C}(23)-\mathrm{C}(24)-\mathrm{N}(4)$ & $-64.8(8)$ \\
\hline $\mathrm{C}(27)-\mathrm{C}(23)-\mathrm{C}(24)-\mathrm{C}(25)$ & $-12.3(7)$ \\
\hline$C(30)-C(23)-C(24)-C(25)$ & $171.4(7)$ \\
\hline $\mathrm{N}(4)-\mathrm{C}(24)-\mathrm{C}(25)-\mathrm{C}(26)$ & $-101.6(7)$ \\
\hline$C(23)-C(24)-C(25)-C(26)$ & $16.0(7)$ \\
\hline$C(24)-C(25)-C(26)-C(27)$ & $-14.1(7)$ \\
\hline $\mathrm{C}(28)-\mathrm{N}(3)-\mathrm{C}(27)-\mathrm{C}(23)$ & $1.4(10)$ \\
\hline $\mathrm{C}(28)-\mathrm{N}(3)-\mathrm{C}(27)-\mathrm{C}(26)$ & $-179.7(7)$ \\
\hline $\mathrm{C}(30)-\mathrm{C}(23)-\mathrm{C}(27)-\mathrm{N}(3)$ & $-0.6(10)$ \\
\hline $\mathrm{C}(24)-\mathrm{C}(23)-\mathrm{C}(27)-\mathrm{N}(3)$ & $-177.2(6)$ \\
\hline$C(30)-C(23)-C(27)-C(26)$ & $-179.6(6)$ \\
\hline$C(24)-C(23)-C(27)-C(26)$ & $3.8(8)$ \\
\hline $\mathrm{C}(25)-\mathrm{C}(26)-\mathrm{C}(27)-\mathrm{N}(3)$ & $-172.4(6)$ \\
\hline
\end{tabular}




\begin{tabular}{|c|c|}
\hline$C(25)-C(26)-C(27)-C(23)$ & $6.6(8)$ \\
\hline $\mathrm{C}(27)-\mathrm{N}(3)-\mathrm{C}(28)-\mathrm{C}(29)$ & $-1.1(10)$ \\
\hline $\mathrm{C}(34)-\mathrm{O}(5)-\mathrm{C}(29)-\mathrm{C}(28)$ & $8.1(10)$ \\
\hline $\mathrm{C}(34)-\mathrm{O}(5)-\mathrm{C}(29)-\mathrm{C}(30)$ & $-171.0(6)$ \\
\hline $\mathrm{N}(3)-\mathrm{C}(28)-\mathrm{C}(29)-\mathrm{O}(5)$ & $-179.0(7)$ \\
\hline $\mathrm{N}(3)-\mathrm{C}(28)-\mathrm{C}(29)-\mathrm{C}(30)$ & $0.1(11)$ \\
\hline $\mathrm{C}(27)-\mathrm{C}(23)-\mathrm{C}(30)-\mathrm{C}(29)$ & $-0.5(10)$ \\
\hline$C(24)-C(23)-C(30)-C(29)$ & $175.5(6)$ \\
\hline$C(27)-C(23)-C(30)-C(31)$ & $175.8(6)$ \\
\hline$C(24)-C(23)-C(30)-C(31)$ & $-8.2(11)$ \\
\hline $\mathrm{O}(5)-\mathrm{C}(29)-\mathrm{C}(30)-\mathrm{C}(23)$ & $180.0(6)$ \\
\hline$C(28)-C(29)-C(30)-C(23)$ & $0.8(10)$ \\
\hline $\mathrm{O}(5)-\mathrm{C}(29)-\mathrm{C}(30)-\mathrm{C}(31)$ & $3.5(9)$ \\
\hline$C(28)-C(29)-C(30)-C(31)$ & $-175.7(6)$ \\
\hline $\mathrm{C}(32)-\mathrm{O}(7)-\mathrm{C}(31)-\mathrm{O}(6)$ & $8.1(10)$ \\
\hline $\mathrm{C}(32)-\mathrm{O}(7)-\mathrm{C}(31)-\mathrm{C}(30)$ & $-172.9(6)$ \\
\hline $\mathrm{C}(23)-\mathrm{C}(30)-\mathrm{C}(31)-\mathrm{O}(6)$ & $120.1(8)$ \\
\hline $\mathrm{C}(29)-\mathrm{C}(30)-\mathrm{C}(31)-\mathrm{O}(6)$ & $-63.6(10)$ \\
\hline $\mathrm{C}(23)-\mathrm{C}(30)-\mathrm{C}(31)-\mathrm{O}(7)$ & $-58.8(8)$ \\
\hline $\mathrm{C}(29)-\mathrm{C}(30)-\mathrm{C}(31)-\mathrm{O}(7)$ & $117.5(7)$ \\
\hline $\mathrm{C}(31)-\mathrm{O}(7)-\mathrm{C}(32)-\mathrm{C}(33)$ & $86.5(8)$ \\
\hline $\mathrm{C}(29)-\mathrm{O}(5)-\mathrm{C}(34)-\mathrm{C}(35)$ & $70.8(8)$ \\
\hline $\mathrm{O}(5)-\mathrm{C}(34)-\mathrm{C}(35)-\mathrm{C}(40)$ & $27.6(10)$ \\
\hline $\mathrm{O}(5)-\mathrm{C}(34)-\mathrm{C}(35)-\mathrm{C}(36)$ & $-154.3(6)$ \\
\hline$C(40)-C(35)-C(36)-C(37)$ & $1.9(10)$ \\
\hline $\mathrm{C}(34)-\mathrm{C}(35)-\mathrm{C}(36)-\mathrm{C}(37)$ & $-176.2(7)$ \\
\hline $\mathrm{C}(35)-\mathrm{C}(36)-\mathrm{C}(37)-\mathrm{C}(38)$ & $-1.8(11)$ \\
\hline $\mathrm{C}(36)-\mathrm{C}(37)-\mathrm{C}(38)-\mathrm{C}(39)$ & $1.4(11)$ \\
\hline$C(37)-C(38)-C(39)-C(40)$ & $-1.3(11)$ \\
\hline $\mathrm{C}(38)-\mathrm{C}(39)-\mathrm{C}(40)-\mathrm{C}(35)$ & $1.4(11)$ \\
\hline$C(36)-C(35)-C(40)-C(39)$ & $-1.7(10)$ \\
\hline$C(34)-C(35)-C(40)-C(39)$ & $176.3(7)$ \\
\hline $\mathrm{O}(8)-\mathrm{S}(2)-\mathrm{C}(41)-\mathrm{C}(44)$ & $63.0(6)$ \\
\hline $\mathrm{N}(4)-\mathrm{S}(2)-\mathrm{C}(41)-\mathrm{C}(44)$ & $-51.1(6)$ \\
\hline $\mathrm{O}(8)-\mathrm{S}(2)-\mathrm{C}(41)-\mathrm{C}(43)$ & $-174.1(6)$ \\
\hline $\mathrm{N}(4)-\mathrm{S}(2)-\mathrm{C}(41)-\mathrm{C}(43)$ & $71.7(6)$ \\
\hline $\mathrm{O}(8)-\mathrm{S}(2)-\mathrm{C}(41)-\mathrm{C}(42)$ & $-55.9(7)$ \\
\hline $\mathrm{N}(4)-\mathrm{S}(2)-\mathrm{C}(41)-\mathrm{C}(42)$ & $-170.1(6)$ \\
\hline
\end{tabular}


Symmetry transformations used to generate equivalent atoms:

Table 7. Hydrogen bonds for mo_(S,R)-8_0m $\quad\left[\AA\right.$ and $\left.^{\circ}\right]$.

D-H...A $\quad$ d(D-H) $\quad$ d(H...A $\quad$ d(D...A $\quad<($ DHA $)$




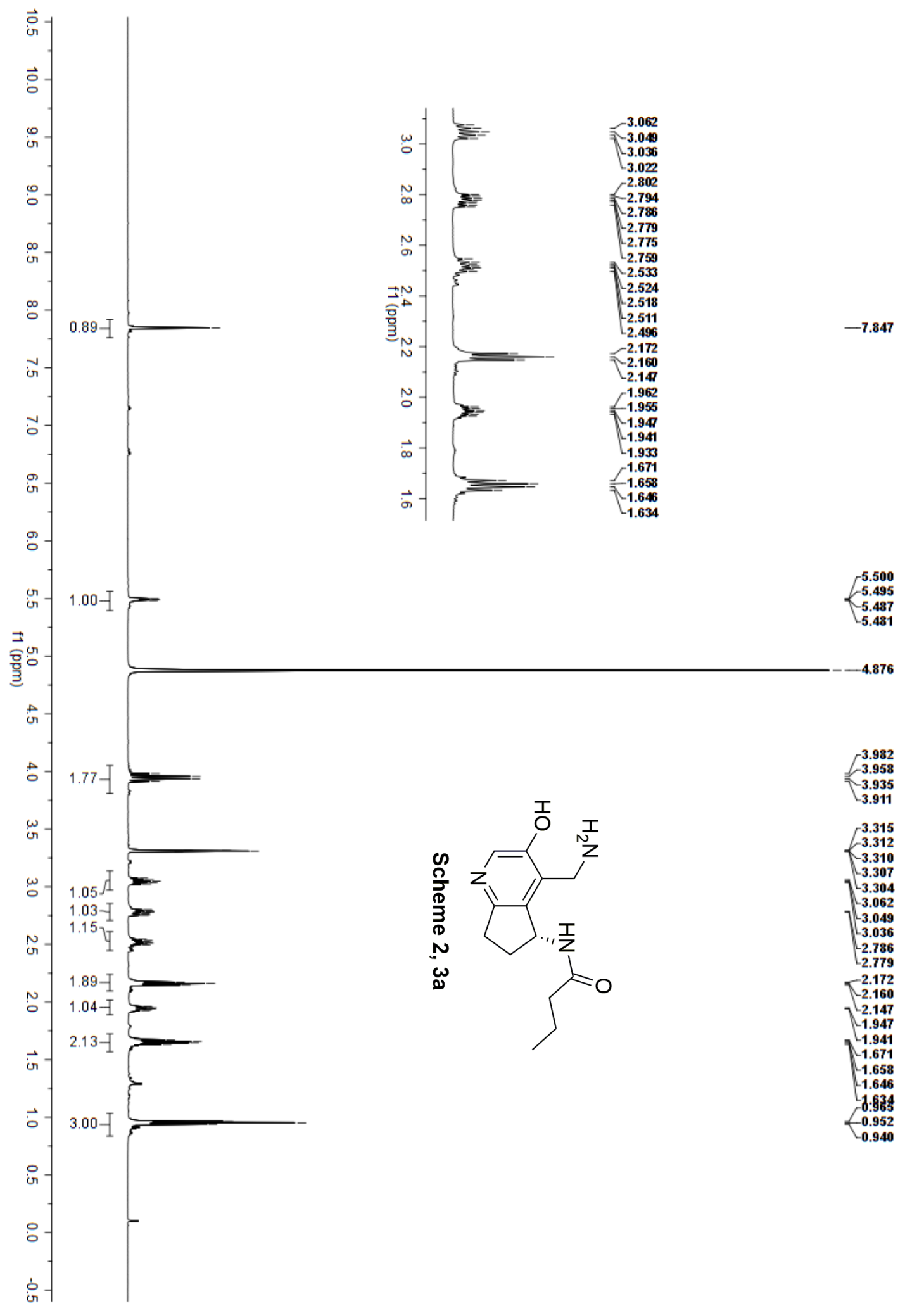




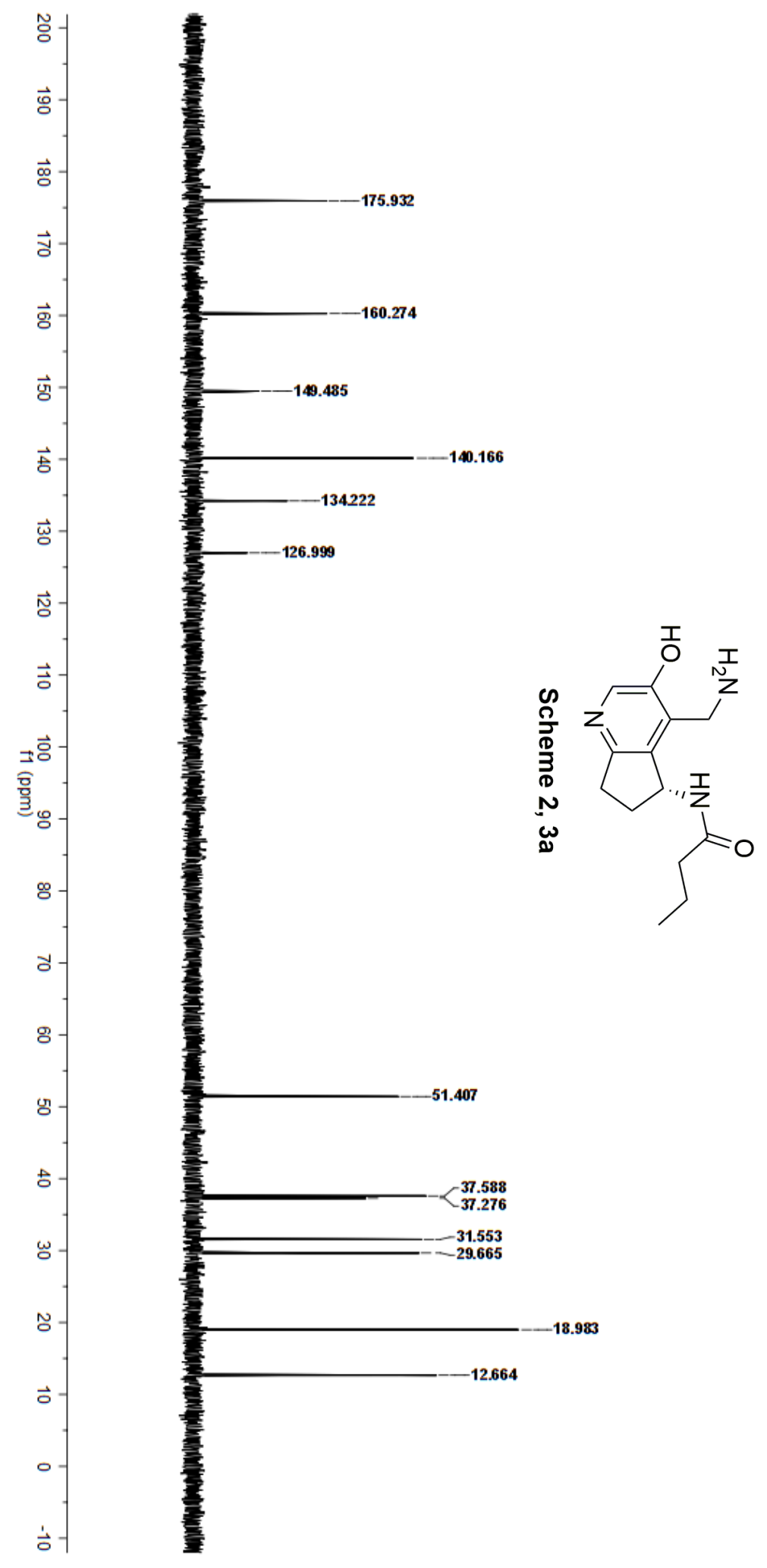



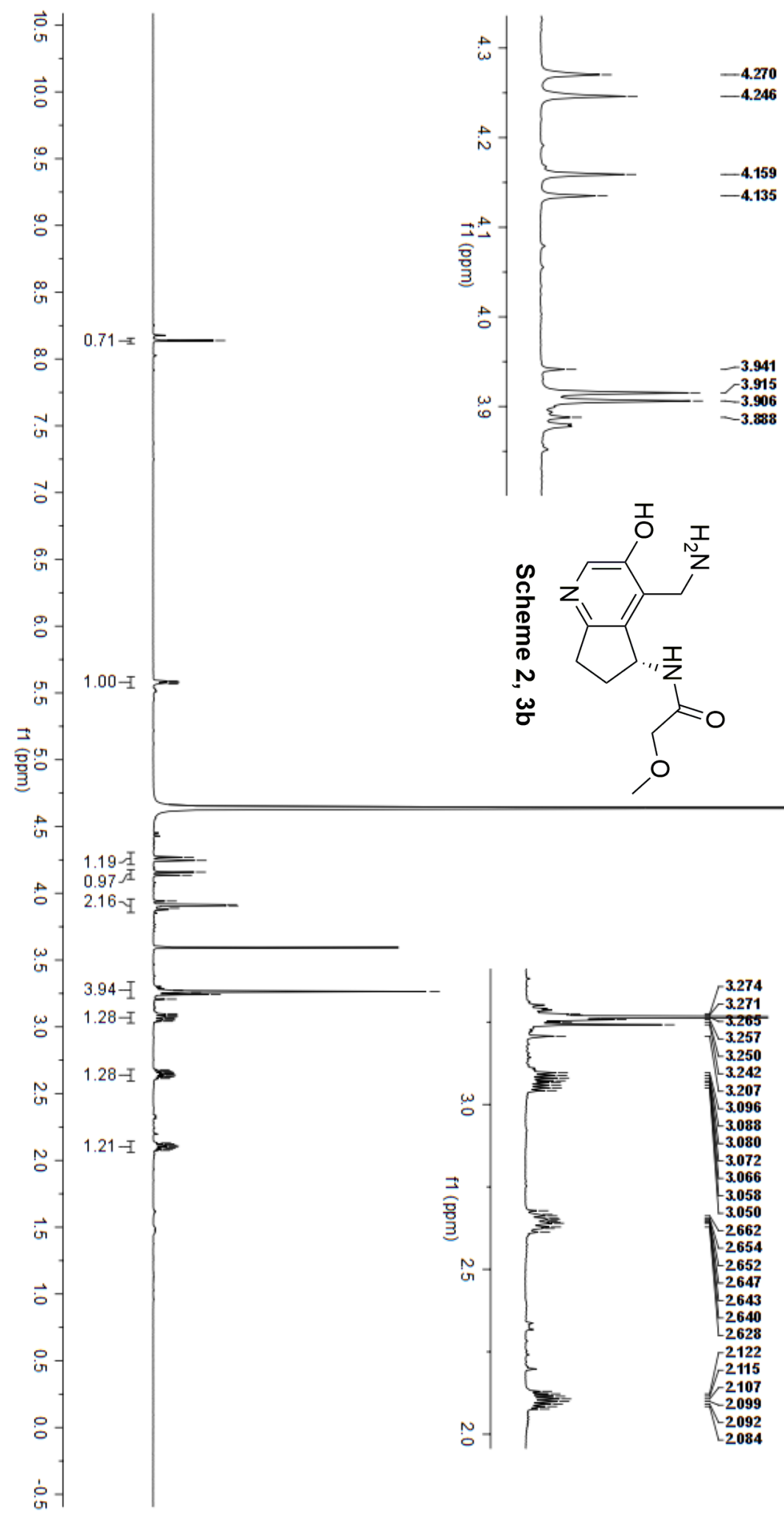

$-8.138$
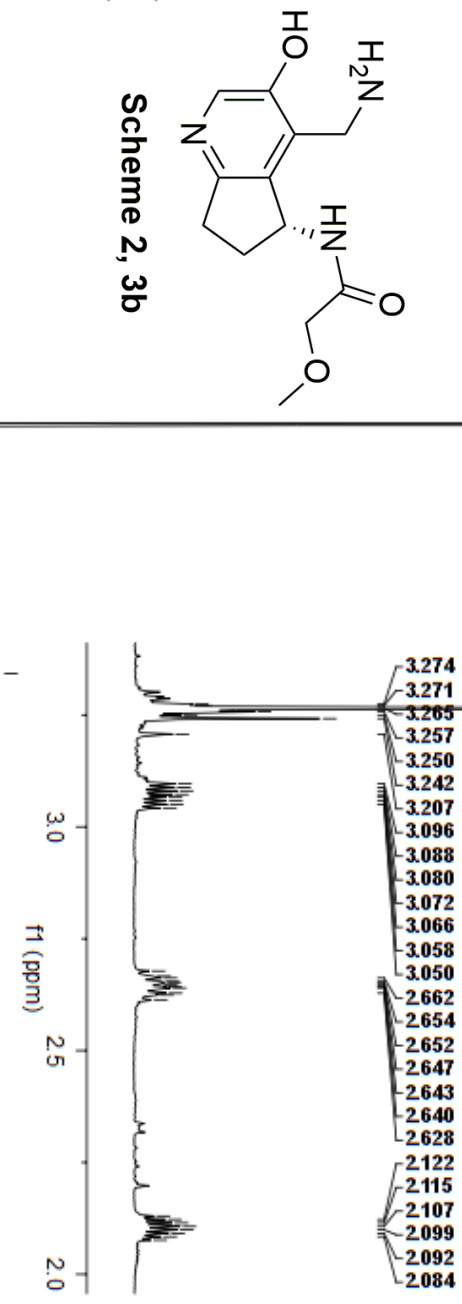

5.590
-5.583

-5.583
-5.576
5.569 

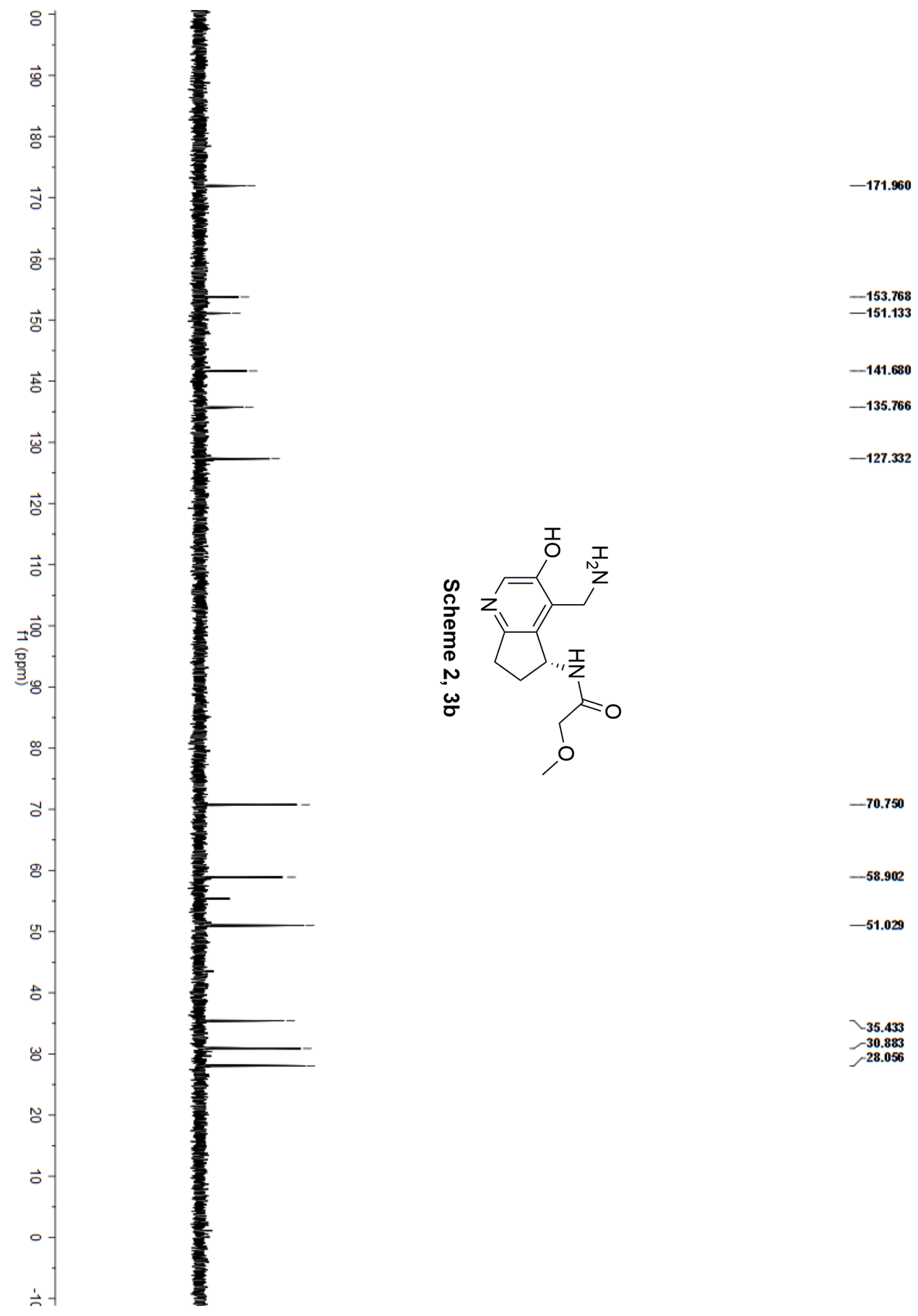

$\checkmark 35.433$ $-\mathbf{- 3 0 . 8 8 3}$ 


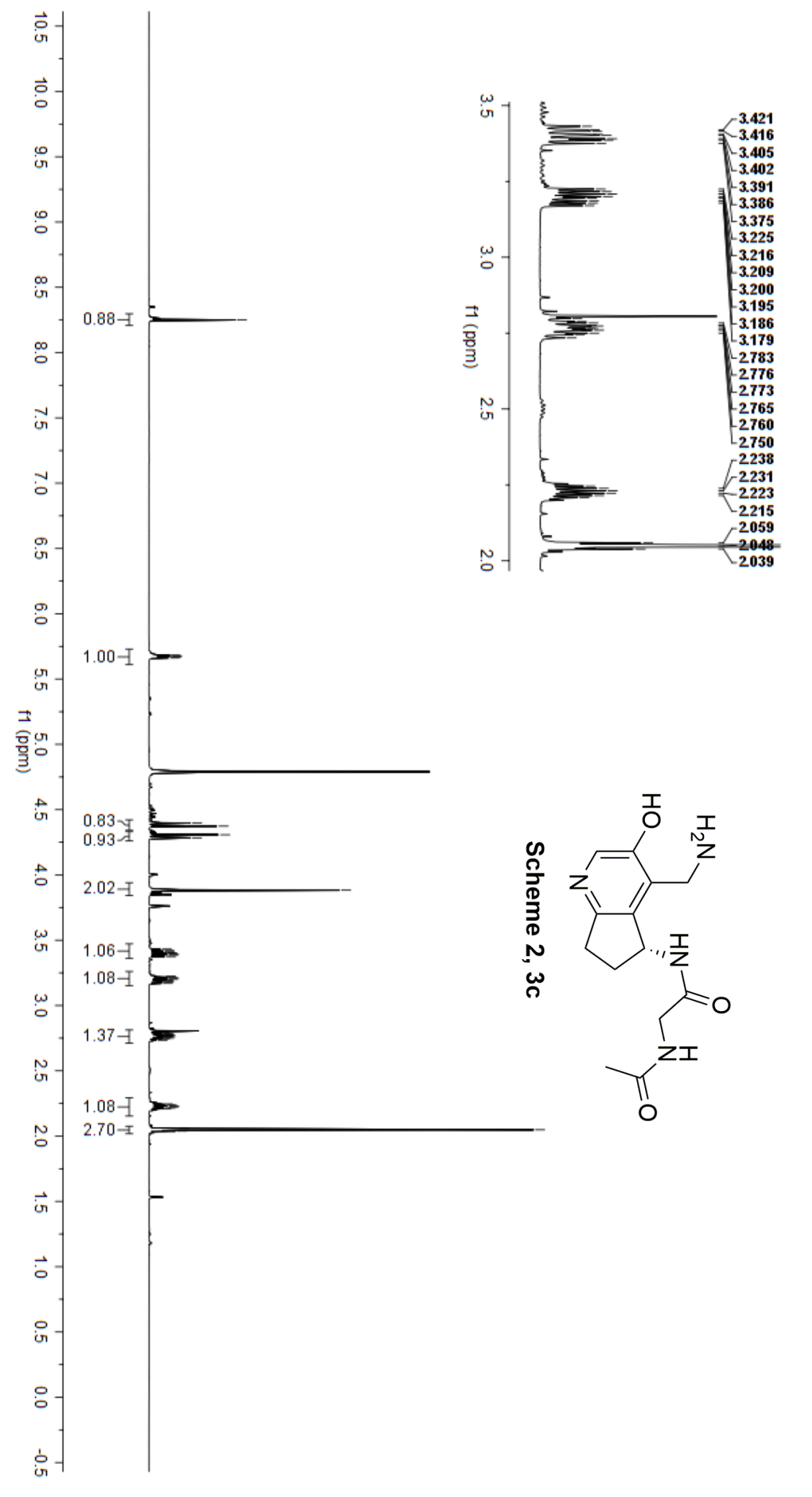




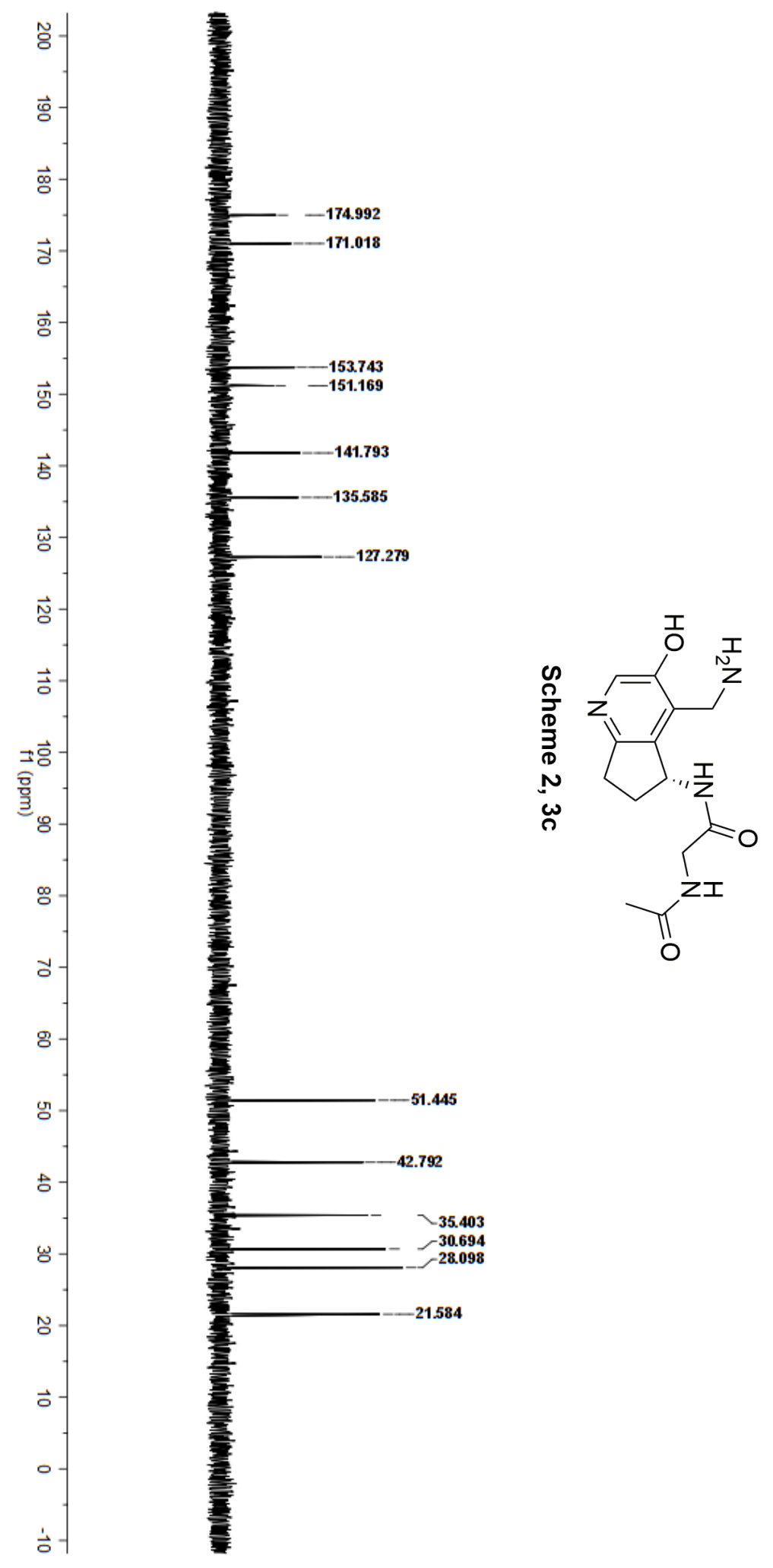




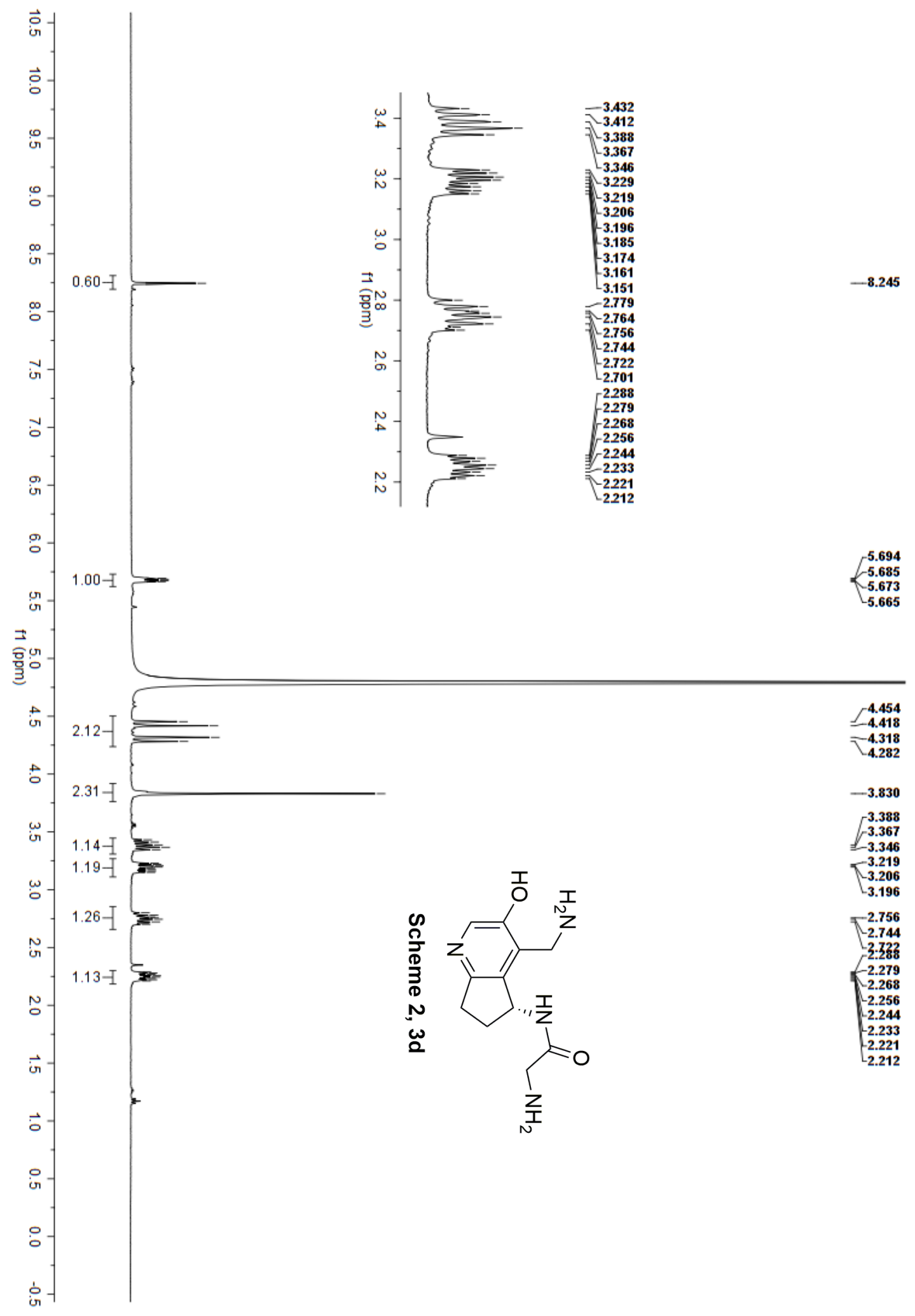




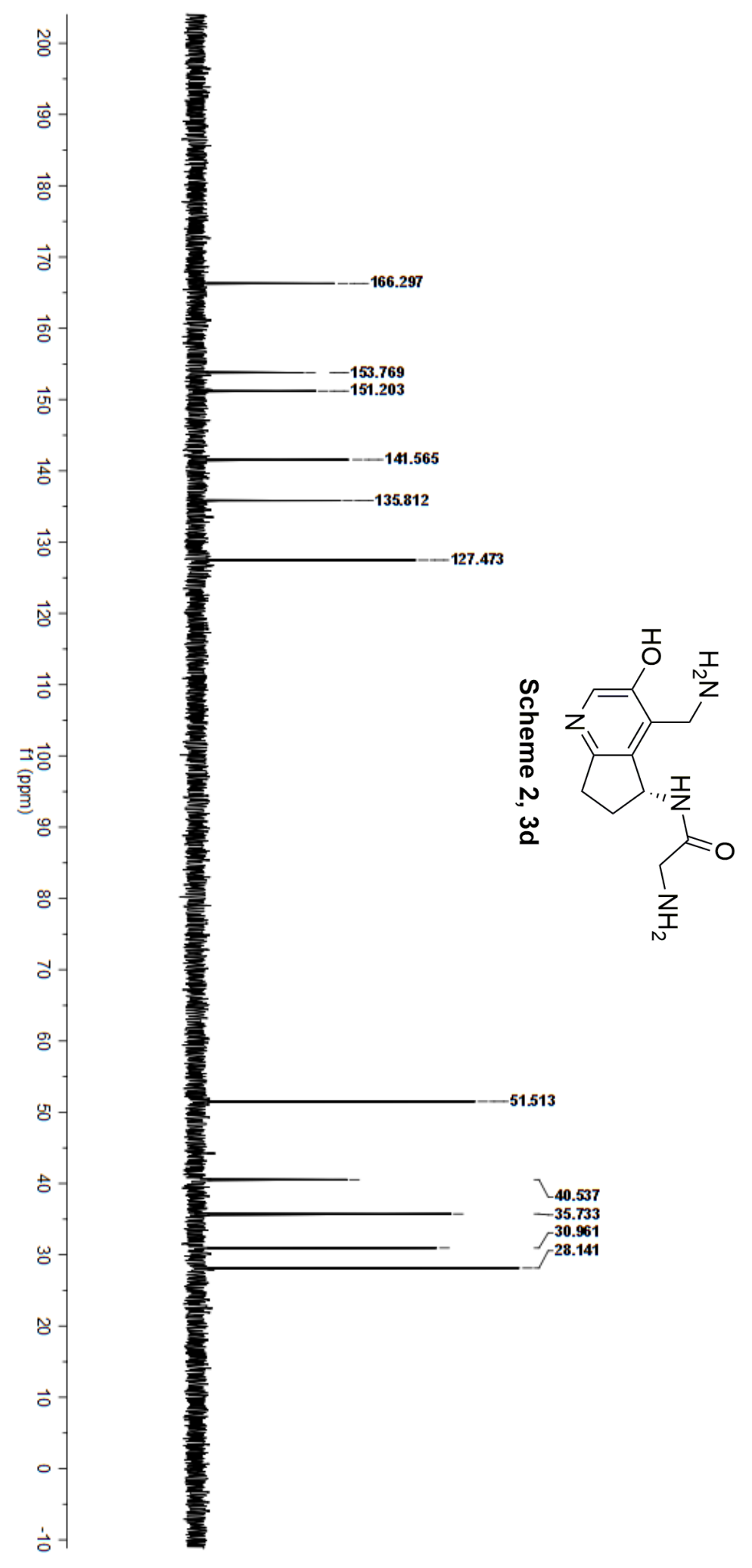




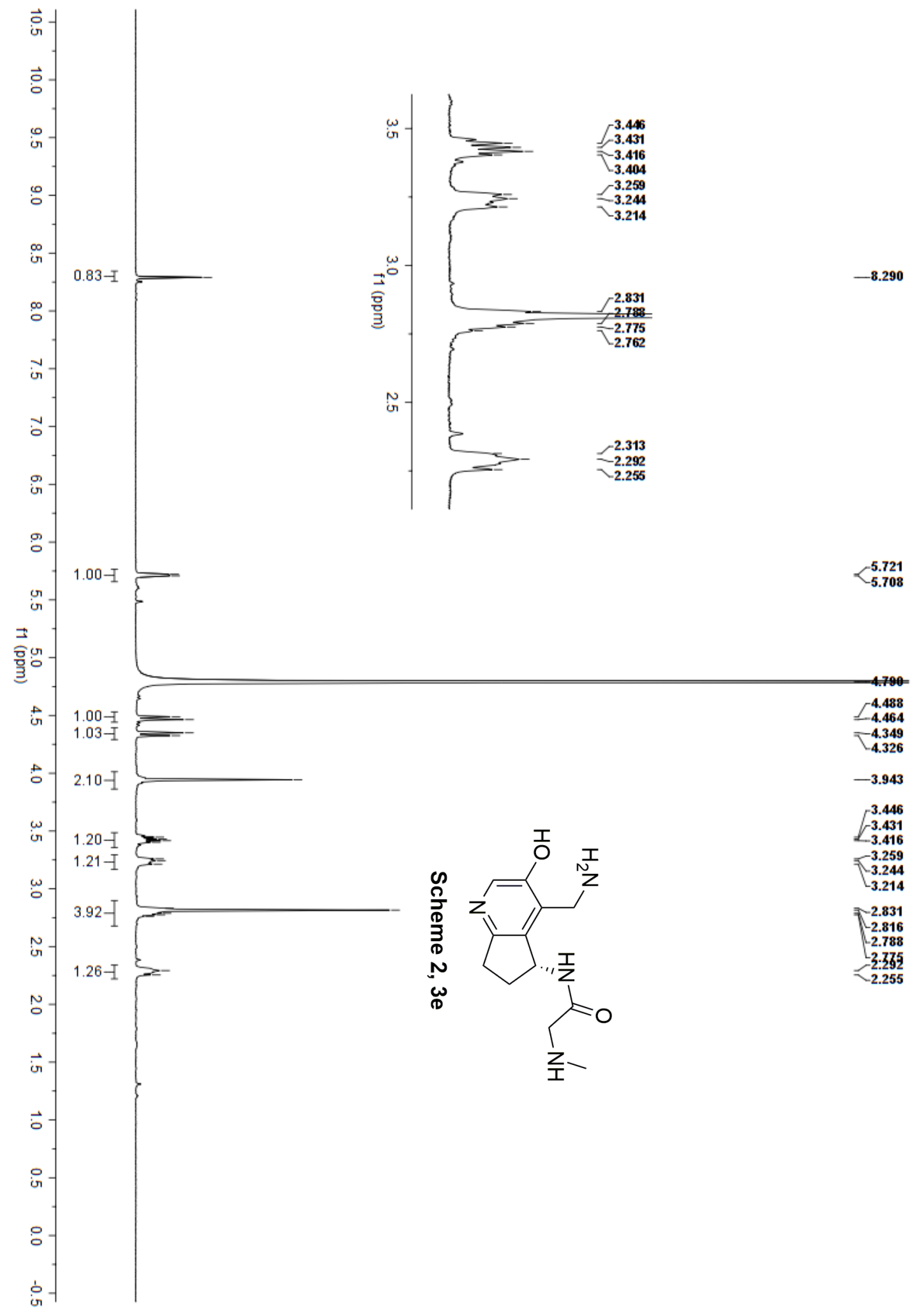




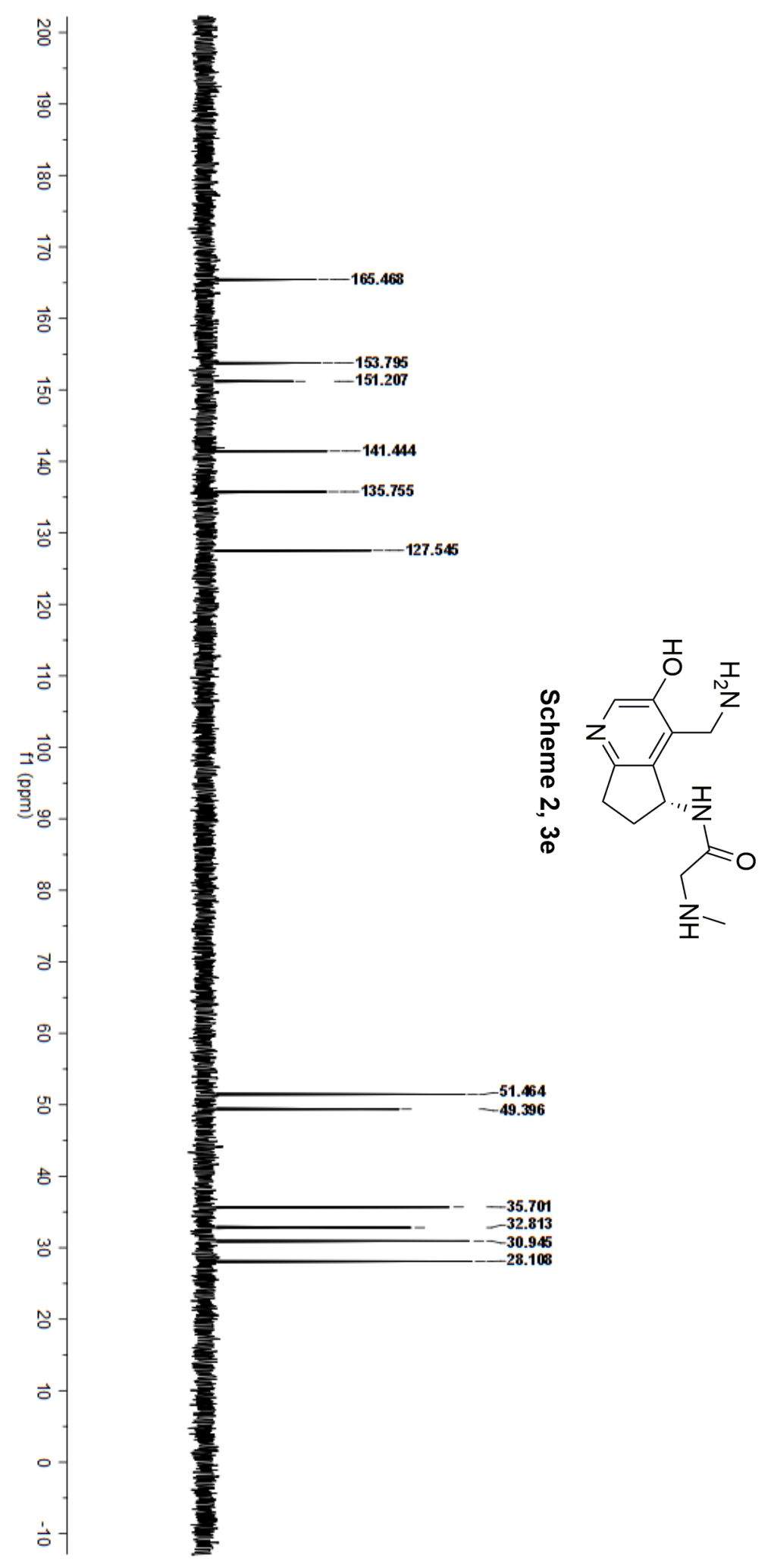




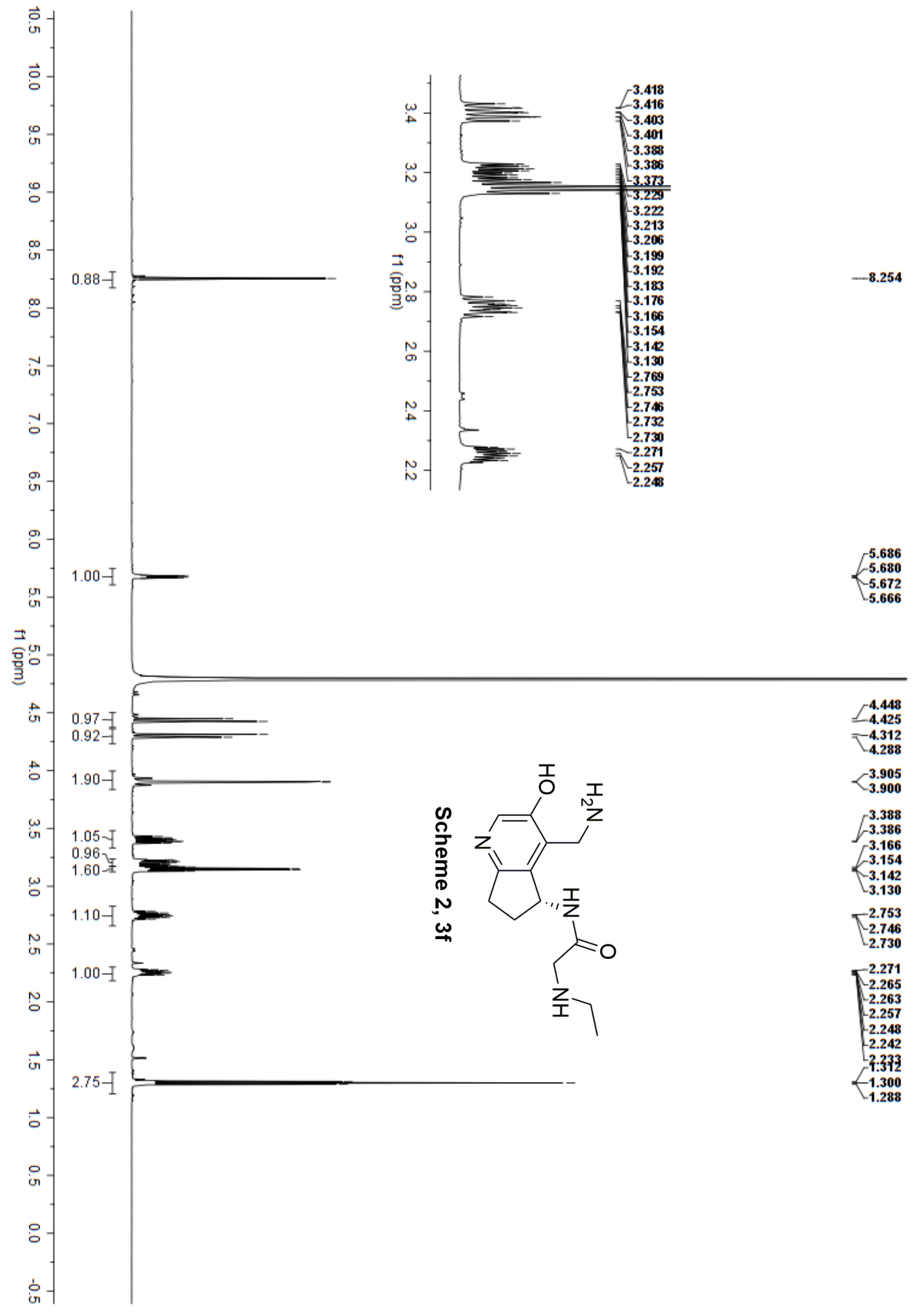




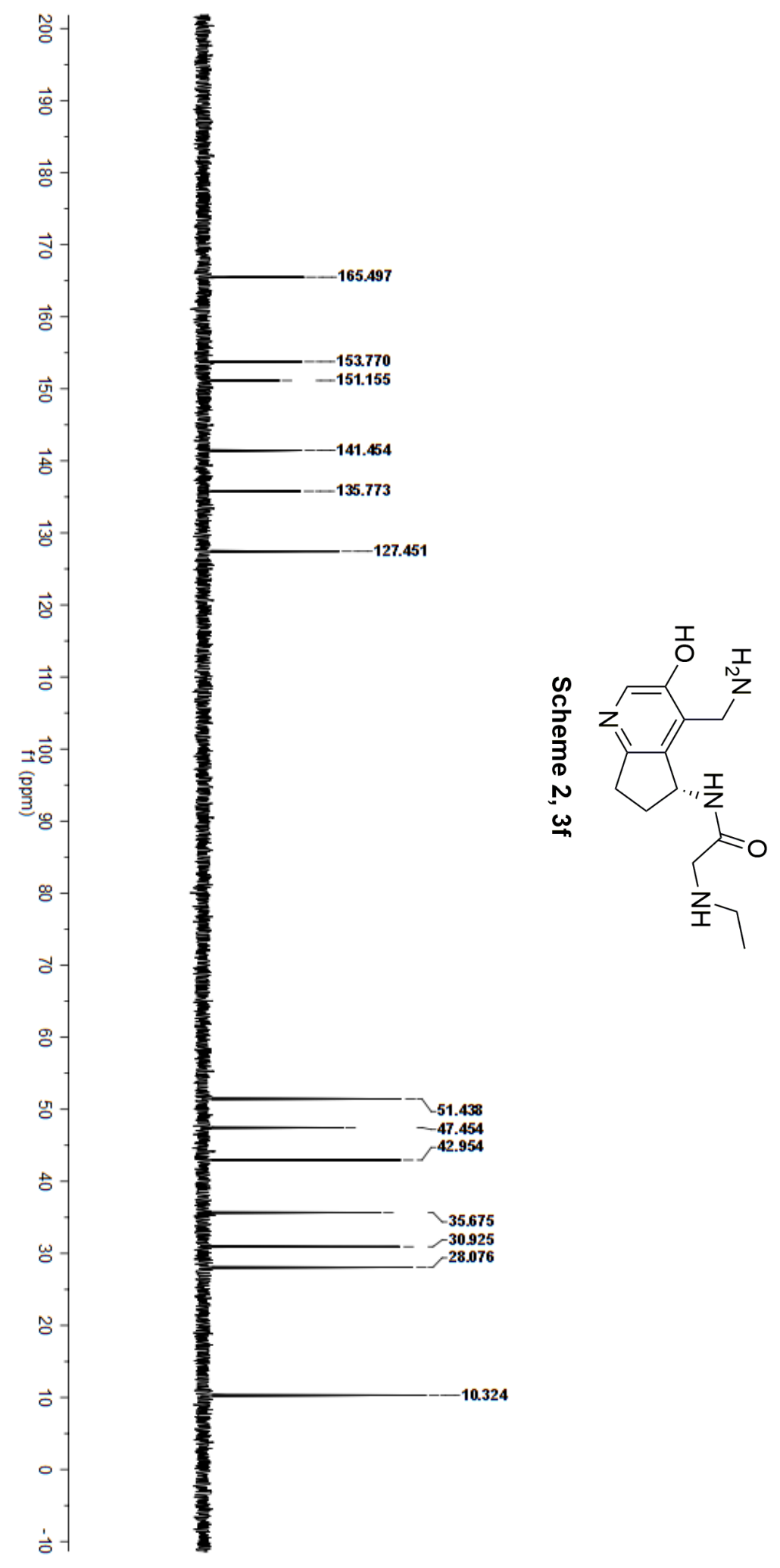




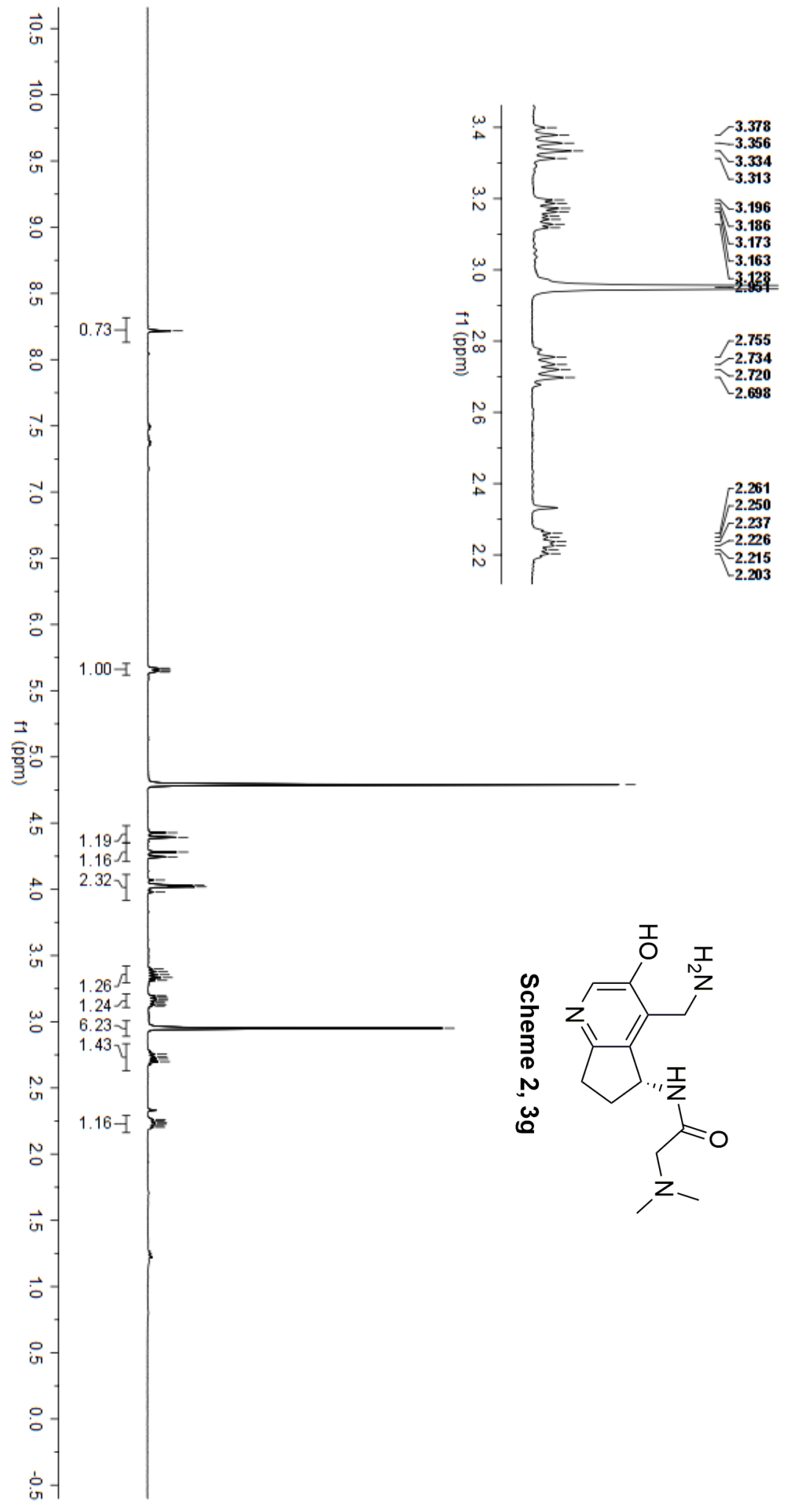

$-\mathbf{8 . 2 1 7}$

5.669
-5.662 5.669
-5.662
-5.649 $\mathbf{5 . 6 4 9}$
$\mathbf{5 . 6 4 1}$ 5.64 $-4.790$ $-4.428$ $-4.392$ $-4.281$ $-4.245$ 4.030
-4.019 -4.019
-3.979 3.378
-3.356 - 3.356 $-3.313$ $-3.313$ $-3.163$ -2.951 -2.951
-2720 -2.720
-2.698 $-2.250$ -2.250
-2.237 2.226
-2.215 -2.226
-2.215
2.203 


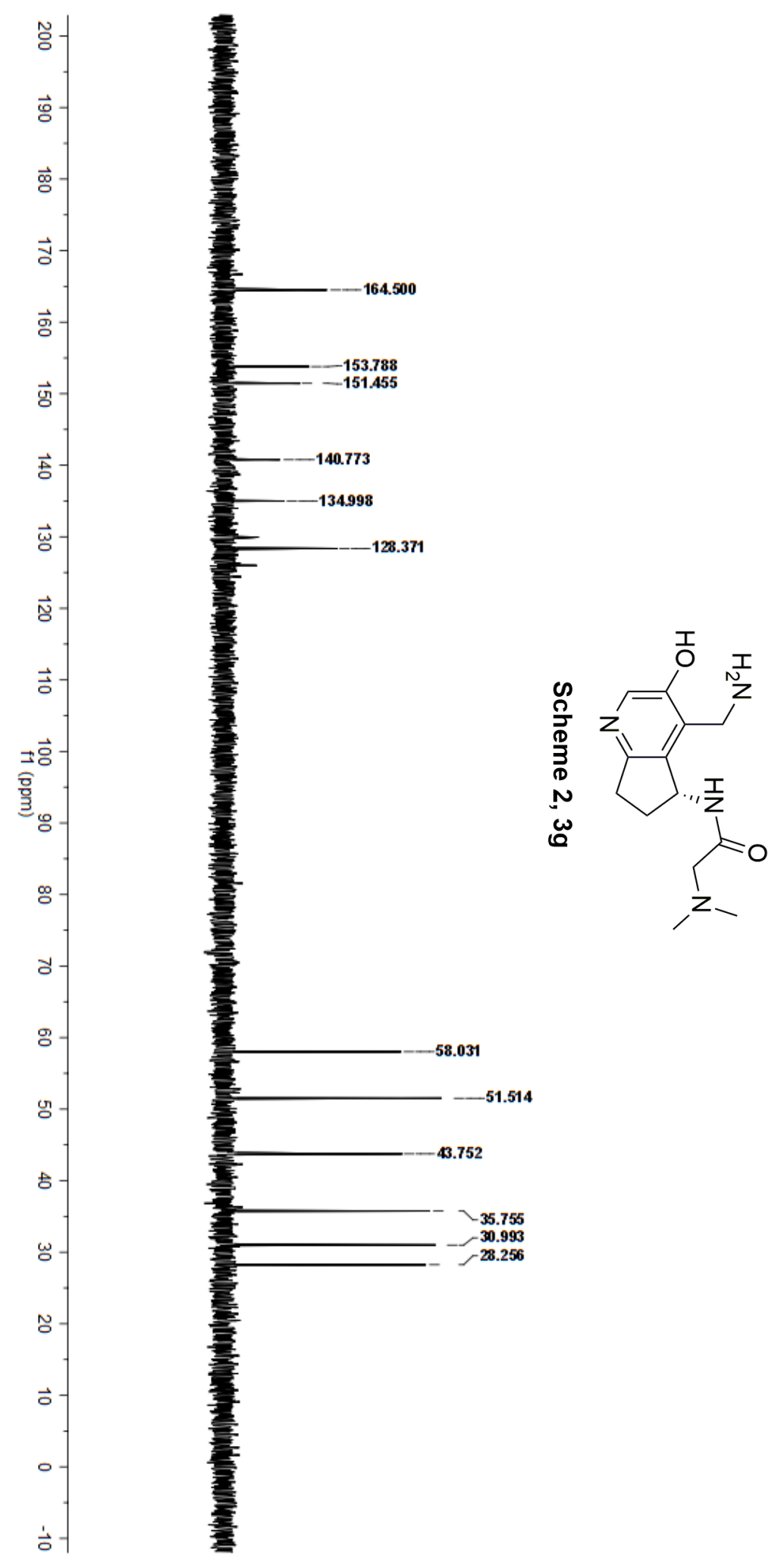




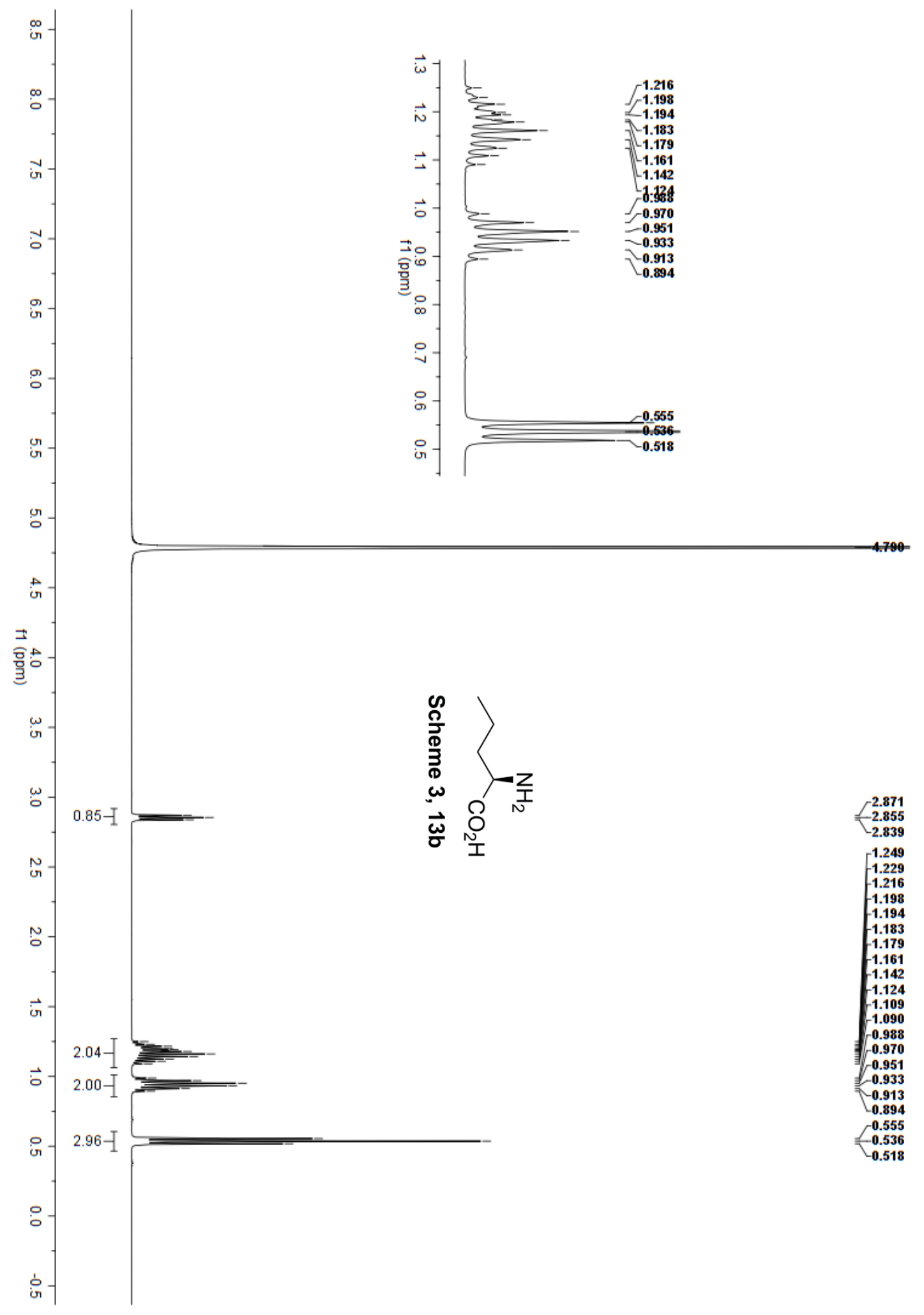




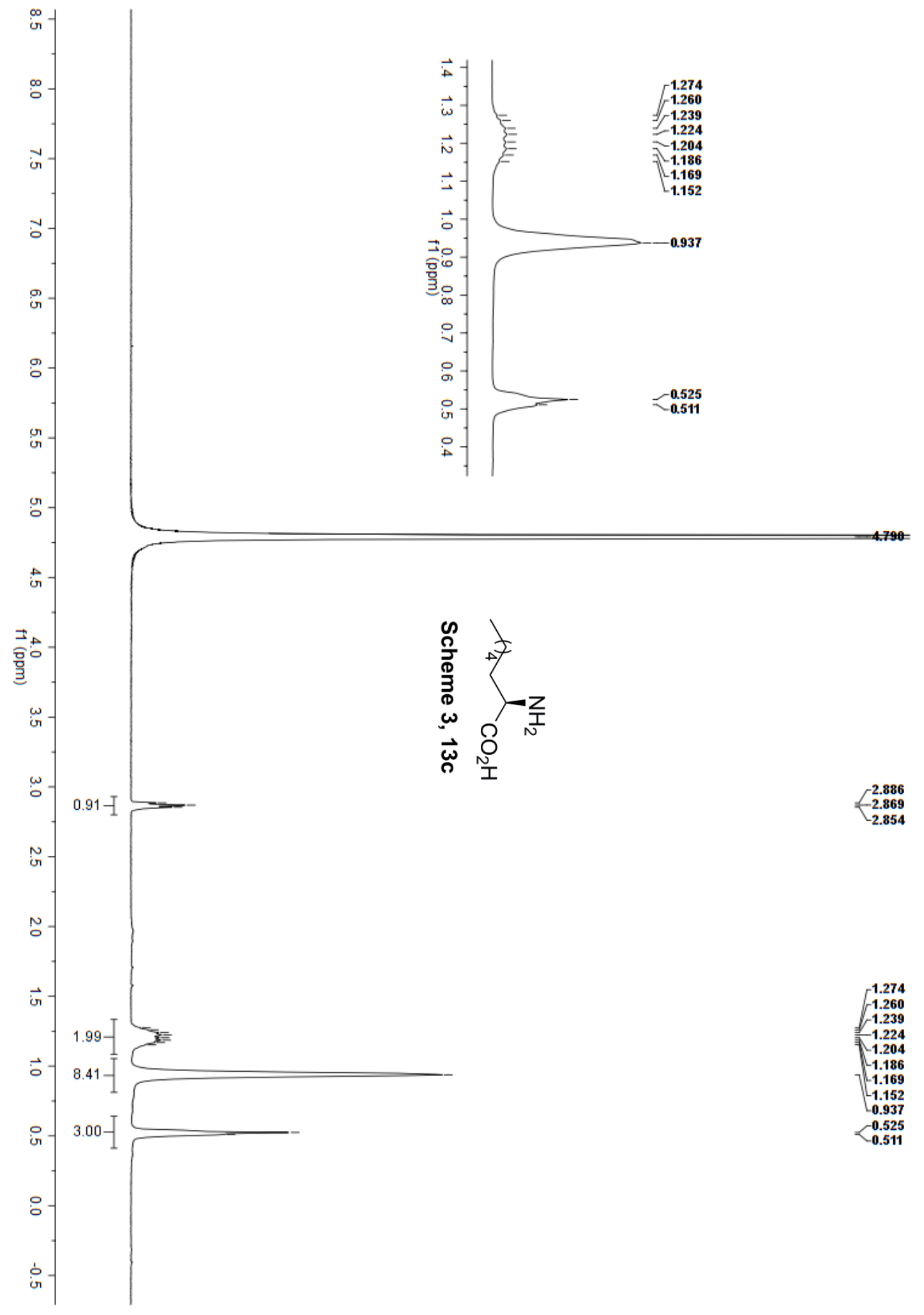




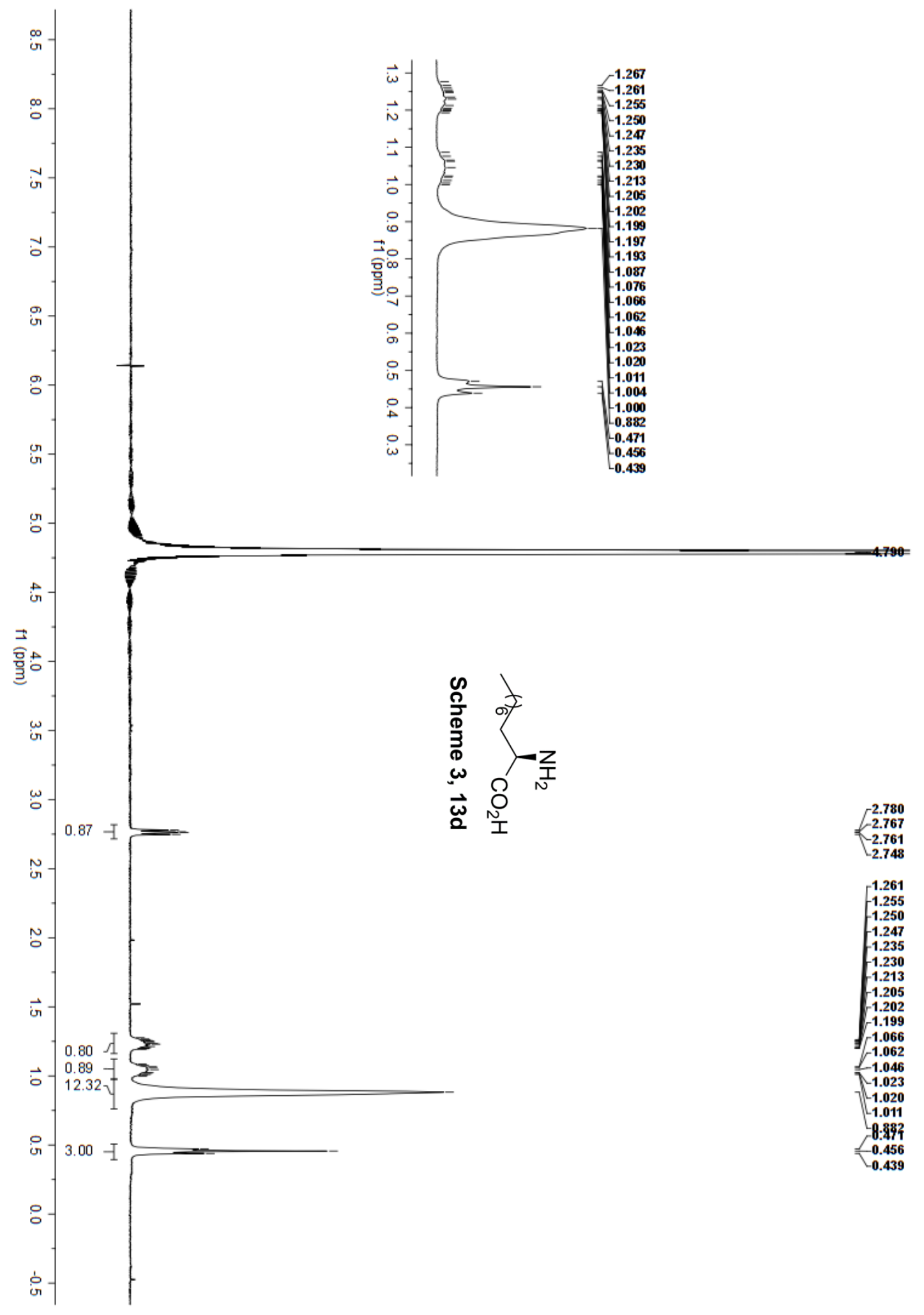




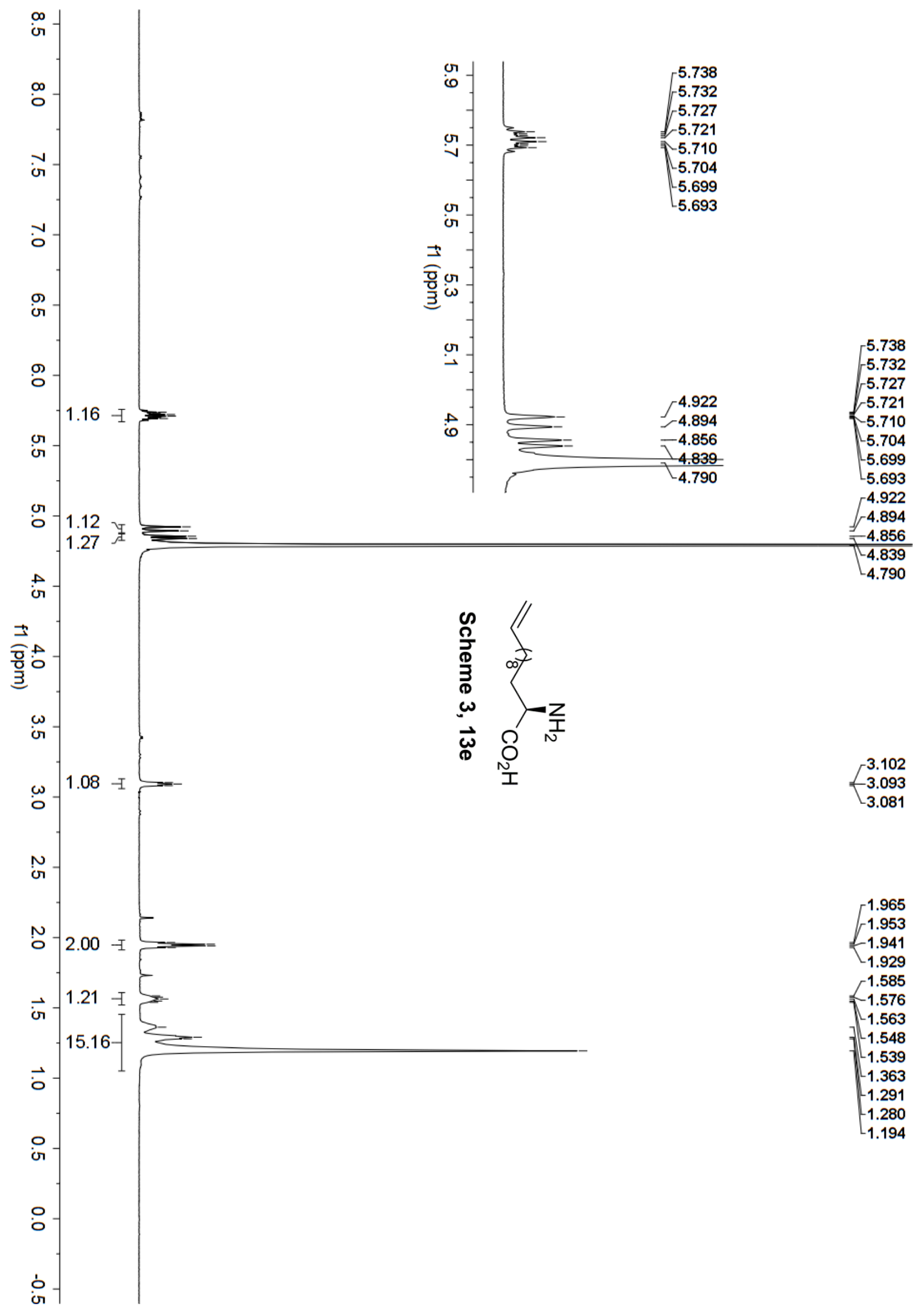



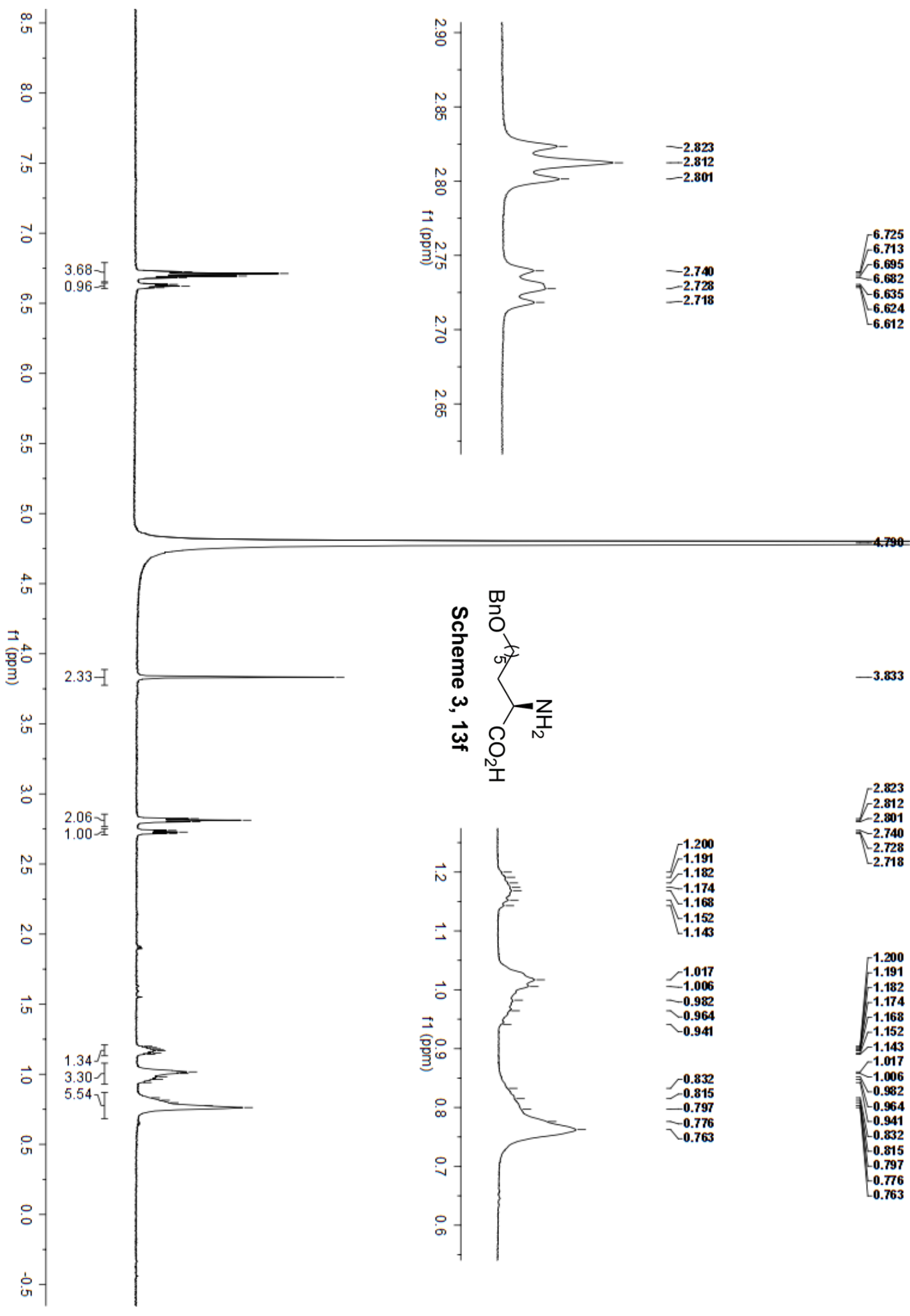

\begin{tabular}{r}
2.823 \\
$L_{-2.812}$ \\
-2.801 \\
\hdashline-2.740 \\
-2.728 \\
2.718
\end{tabular}

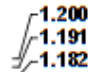

1.191
-1.182
-1.174

$-1.174$

-1.168
-1.152

-1.152
1.143

$-3.833$

$-1.017$

-1.017
$-\mathbf{1 . 0 0 6}$

$-0.982$

$-0.964$

-0.832
-0.815

$-0.797$

-0.776
-0.763

1.200
-1.191
-1.182
-1.174
-1.168
-1.152
-1.143
-1.017
-1.006
0.982
-0.964
0.941
-0.832
-0.815
-0.797
-0.776
0.763 


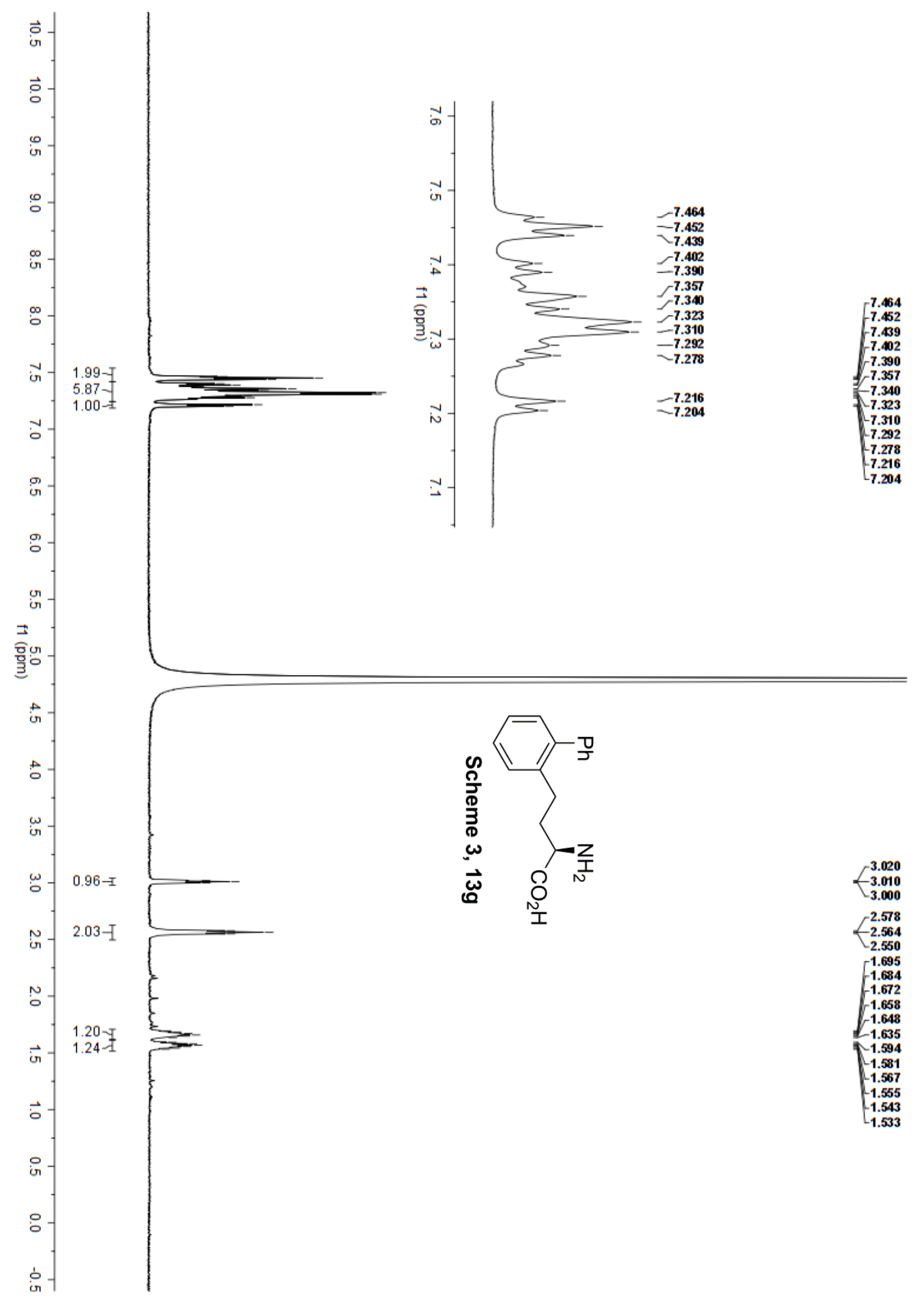




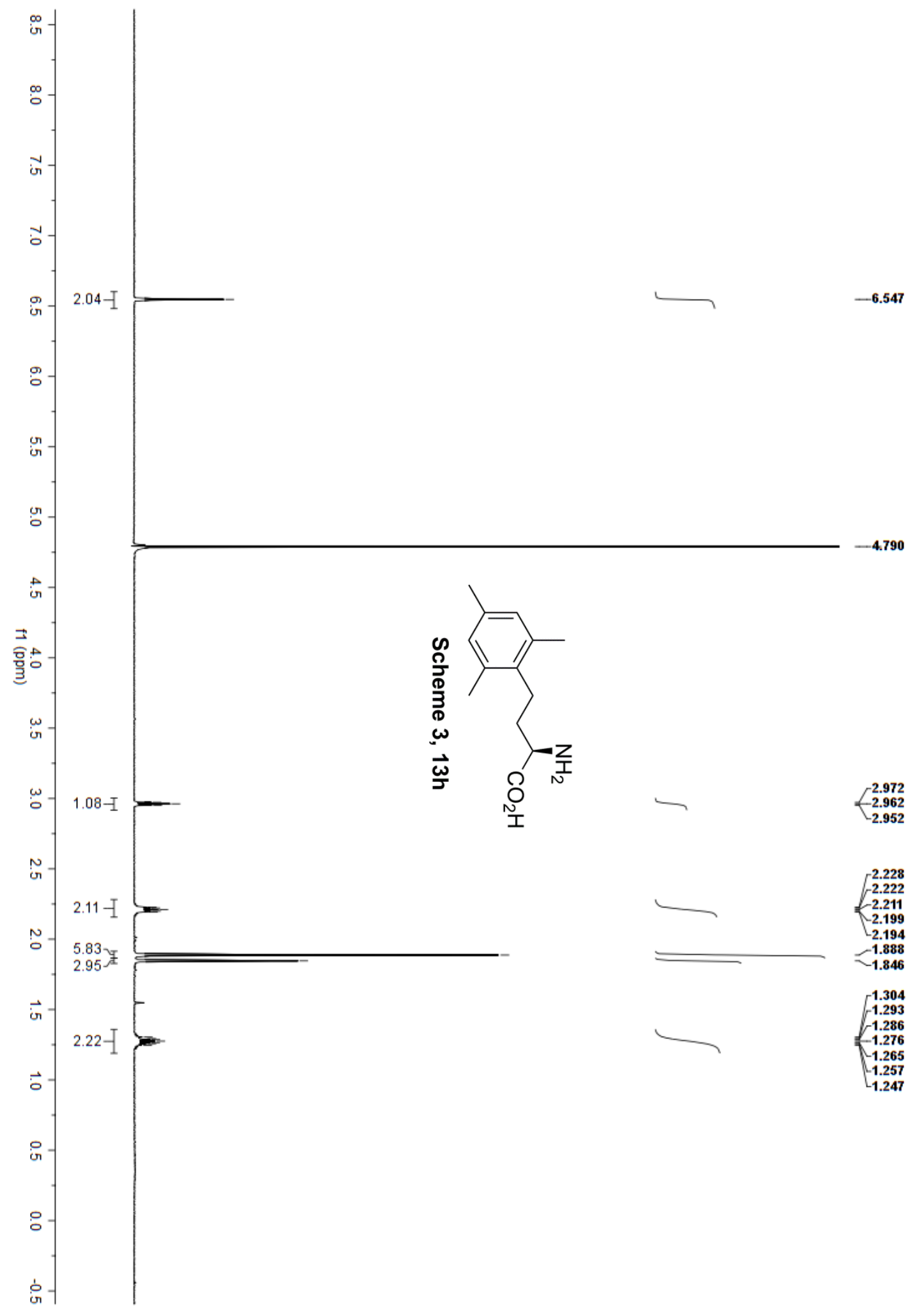




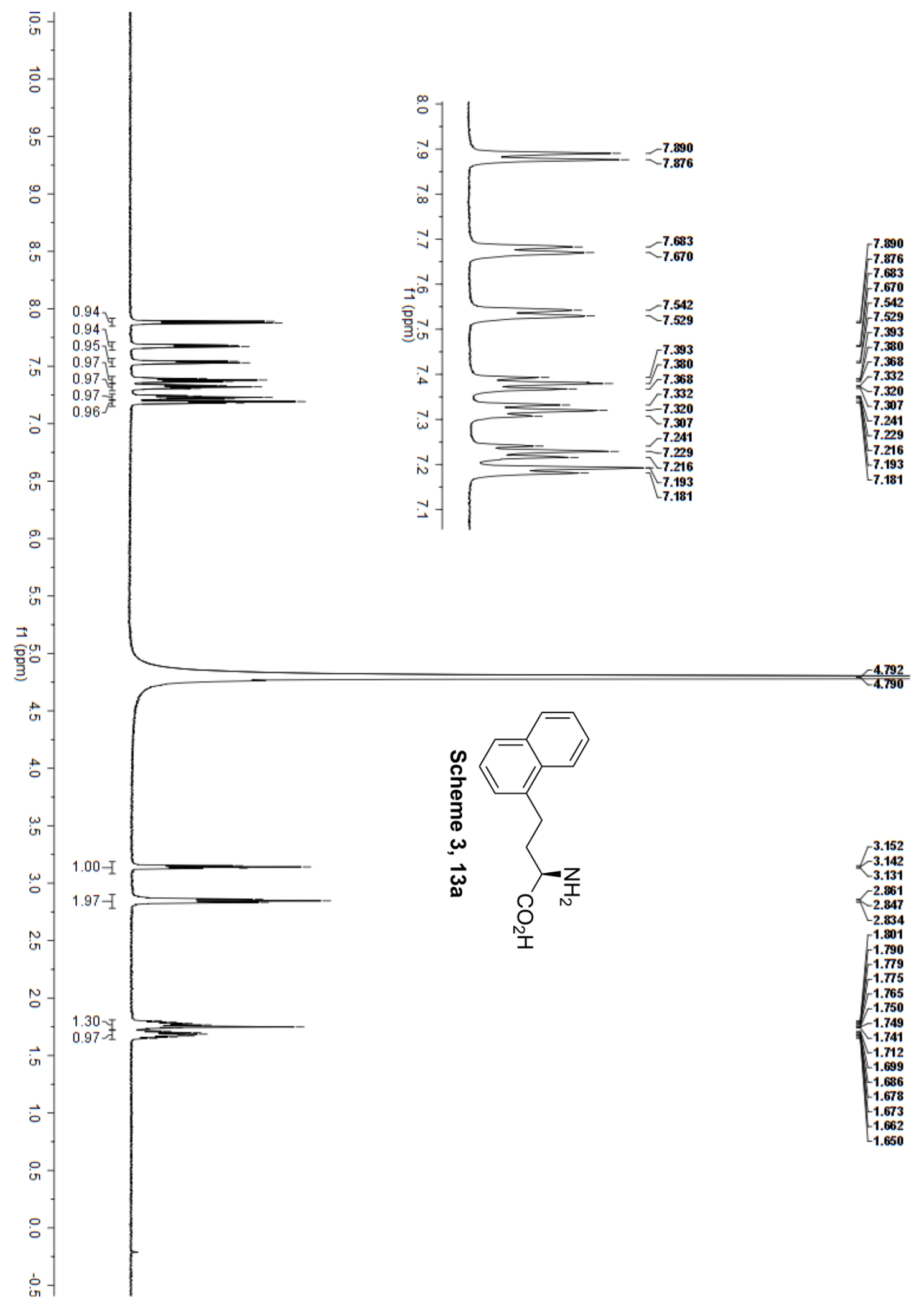




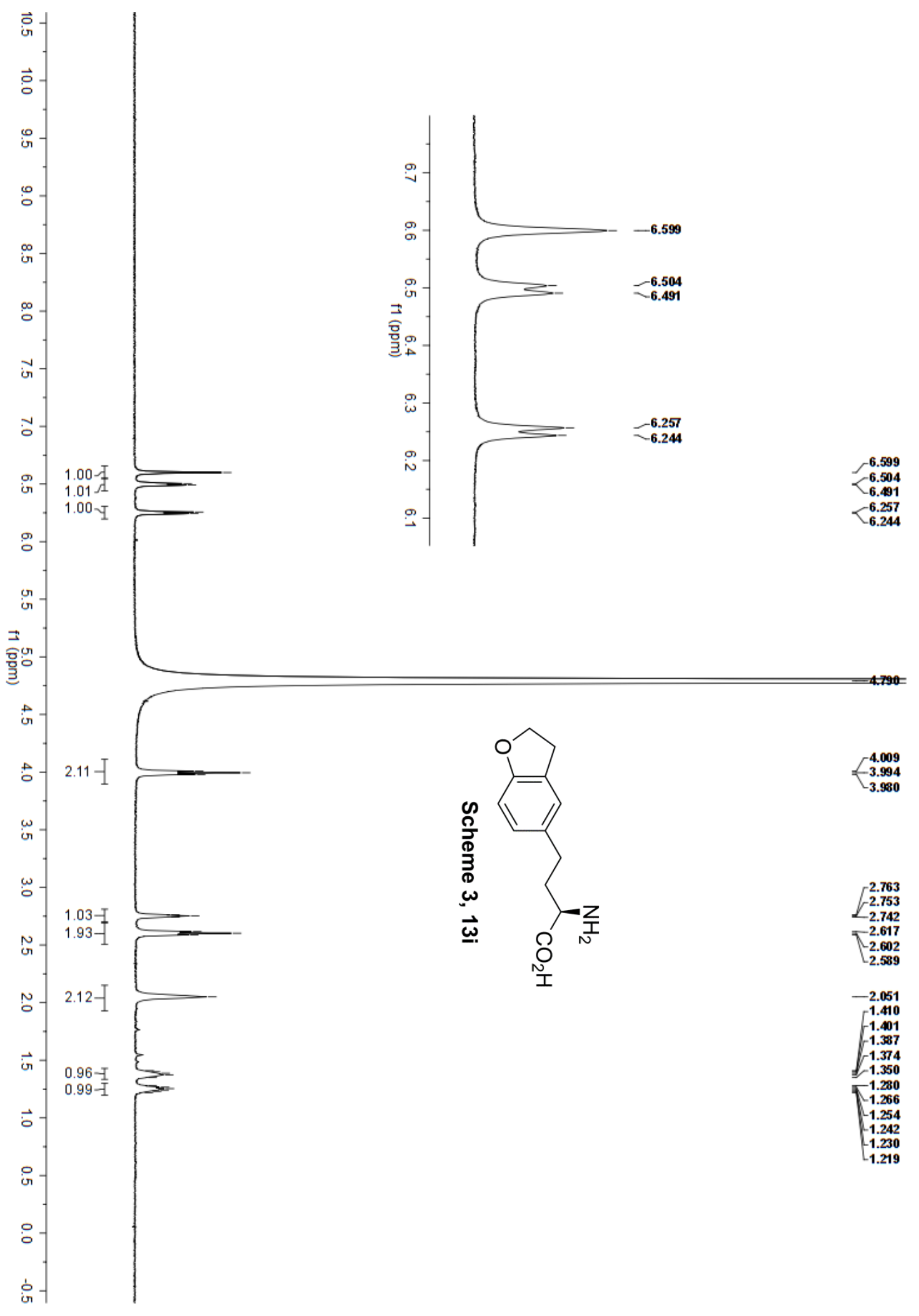




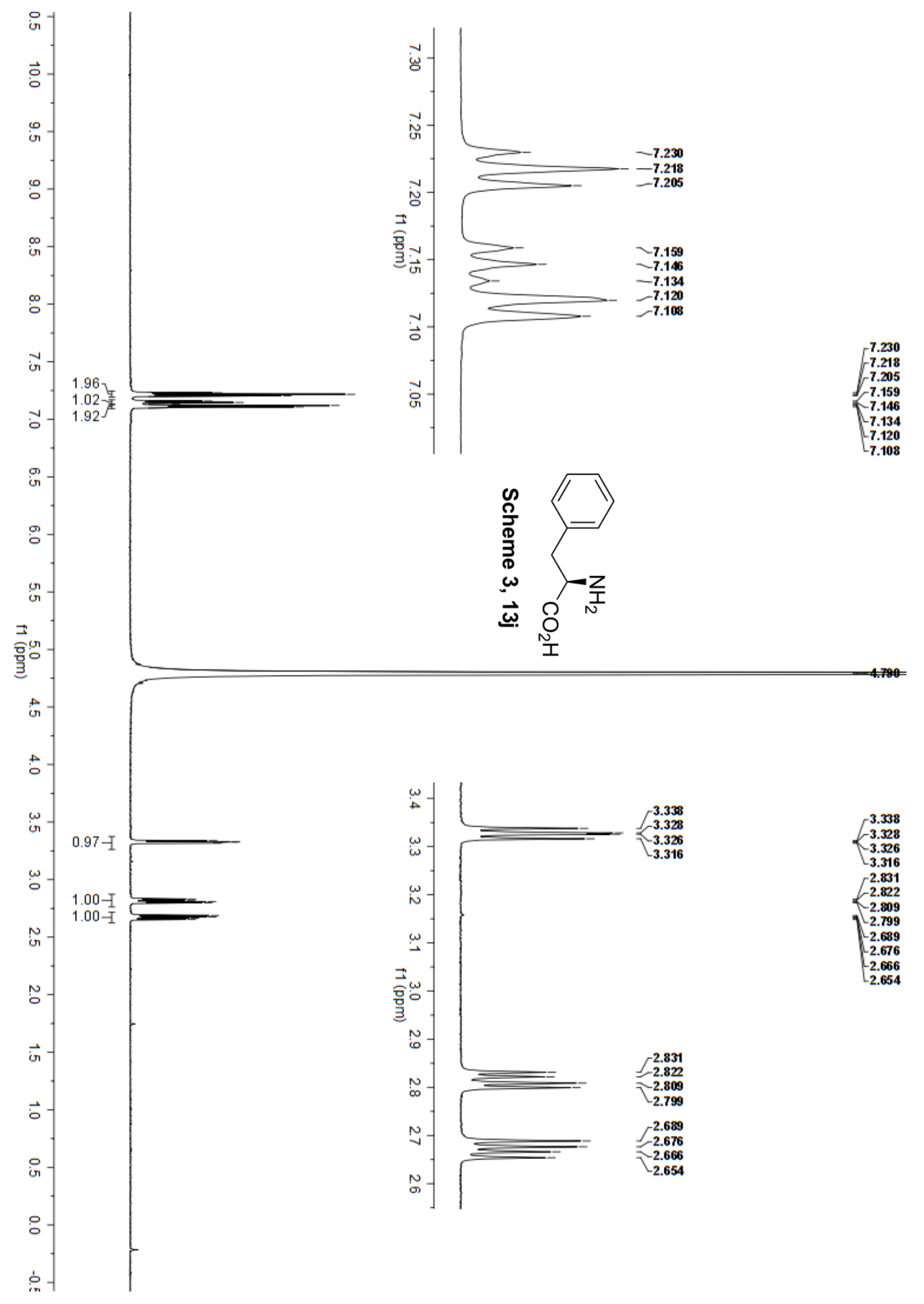




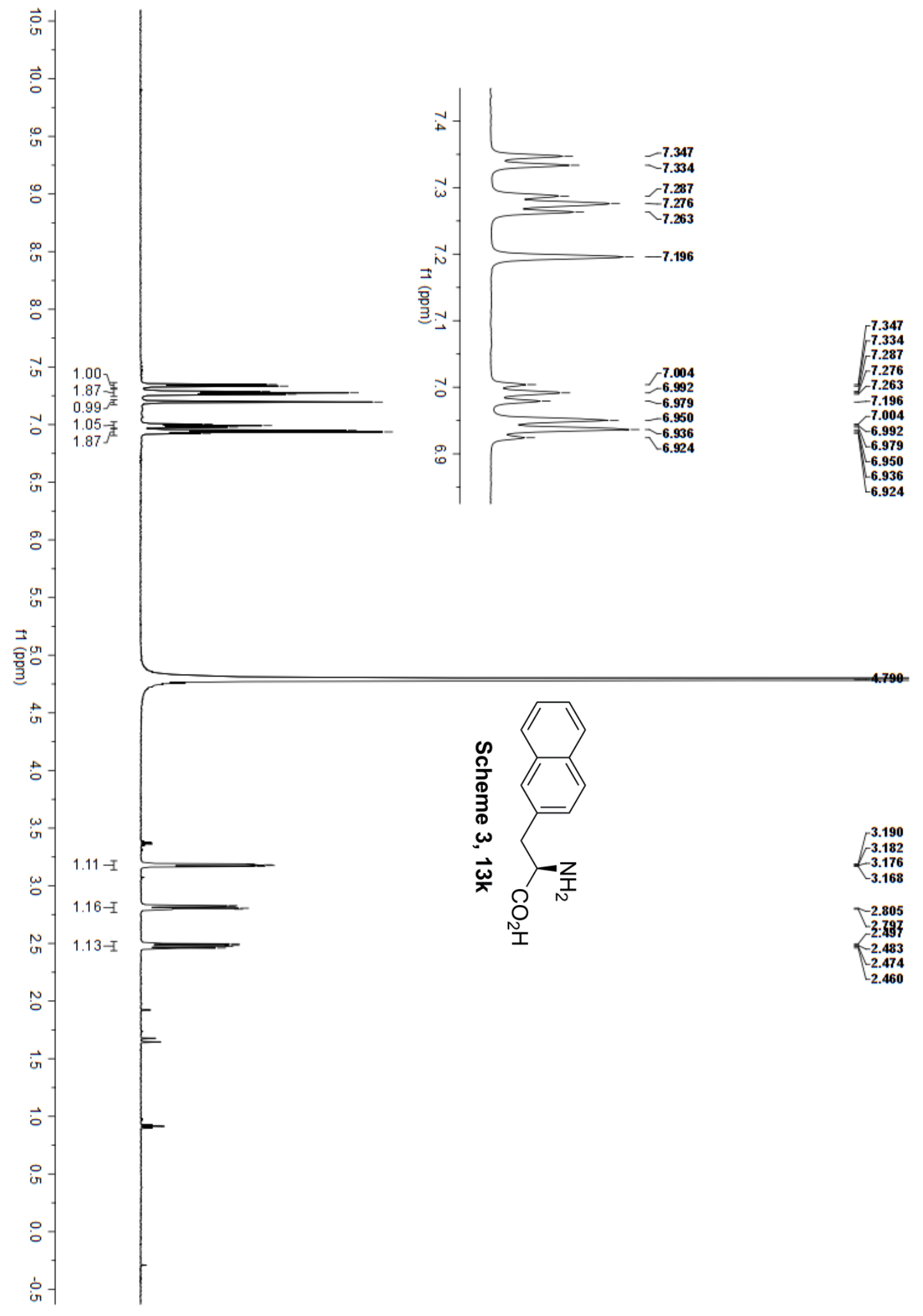




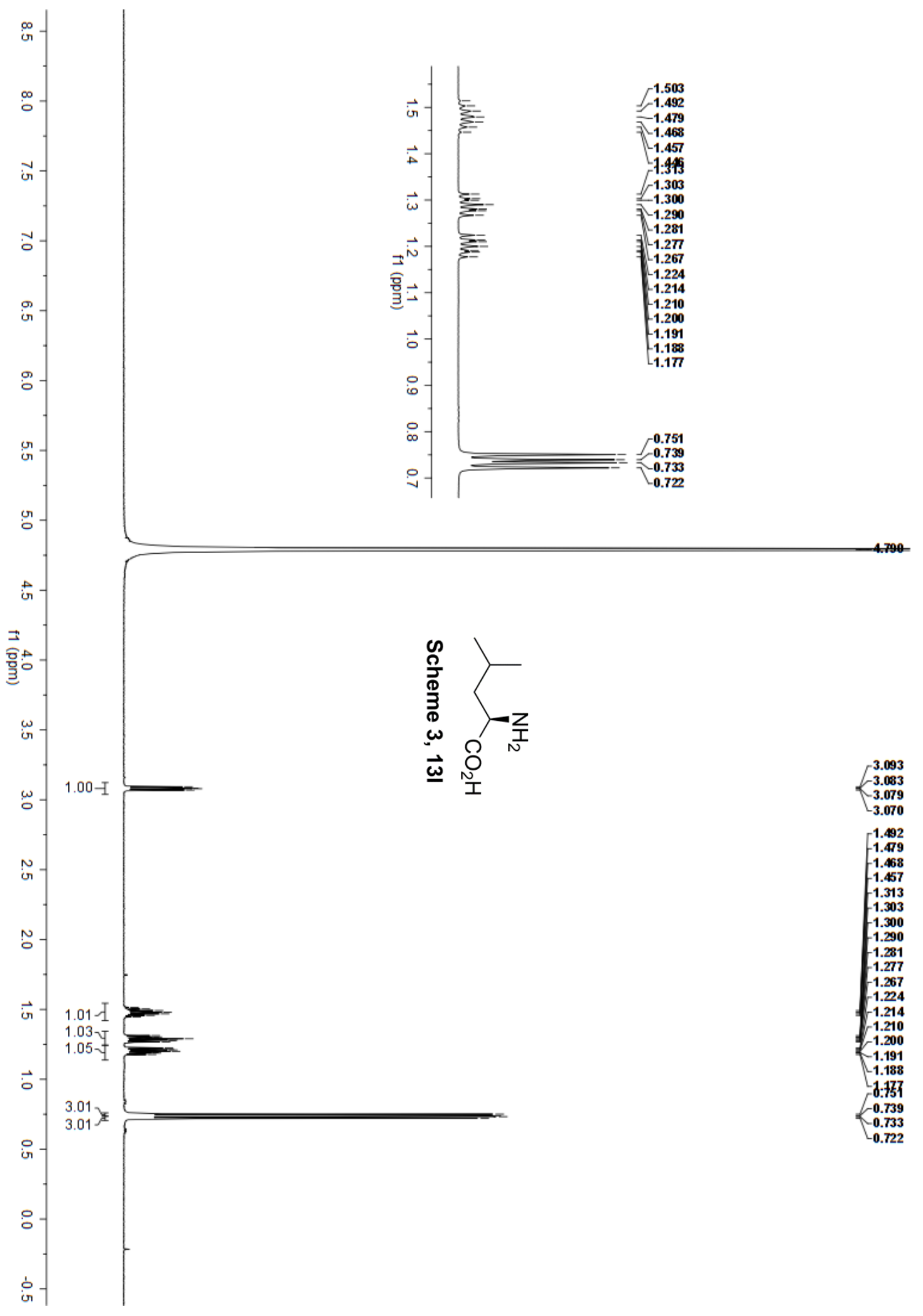




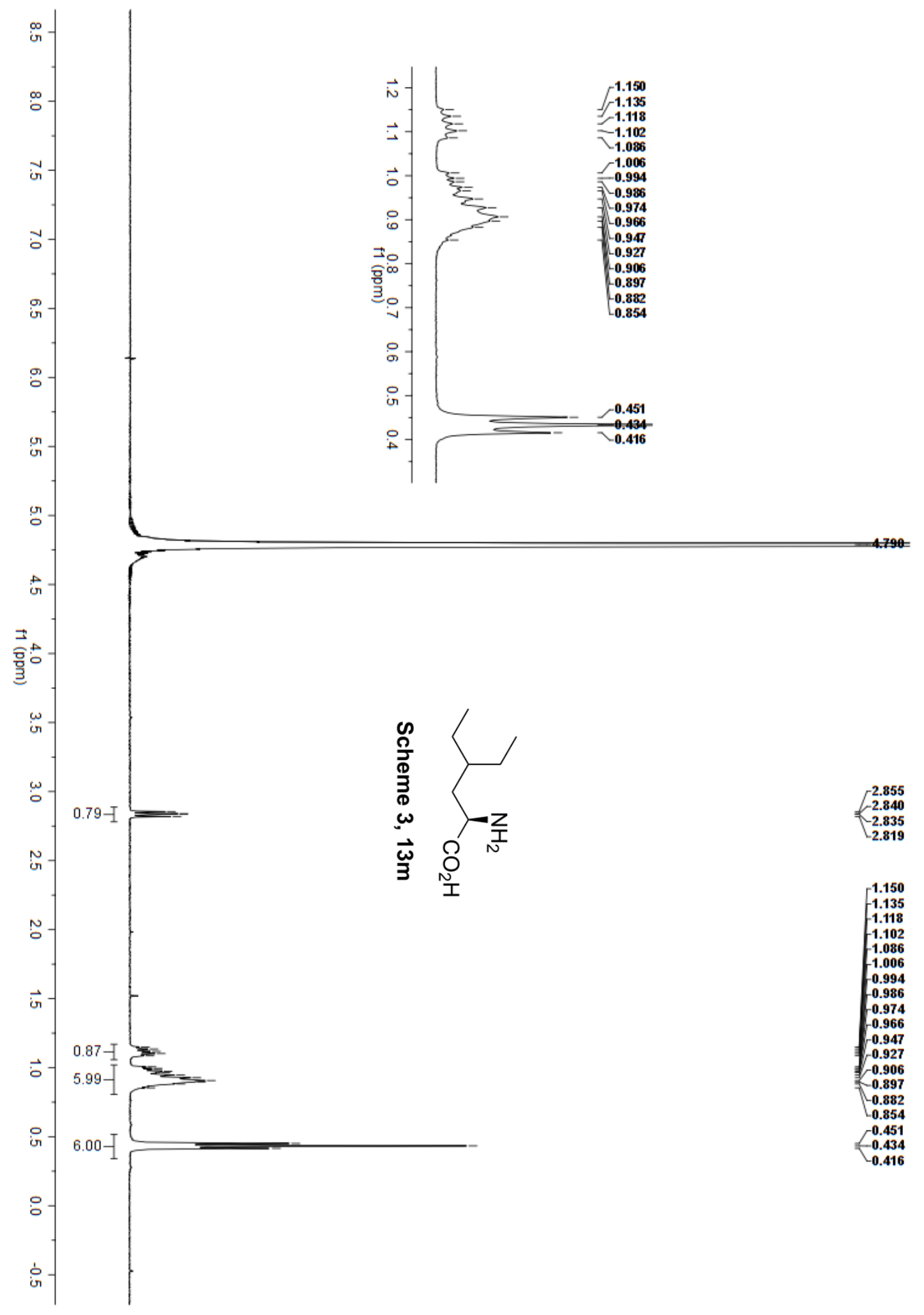




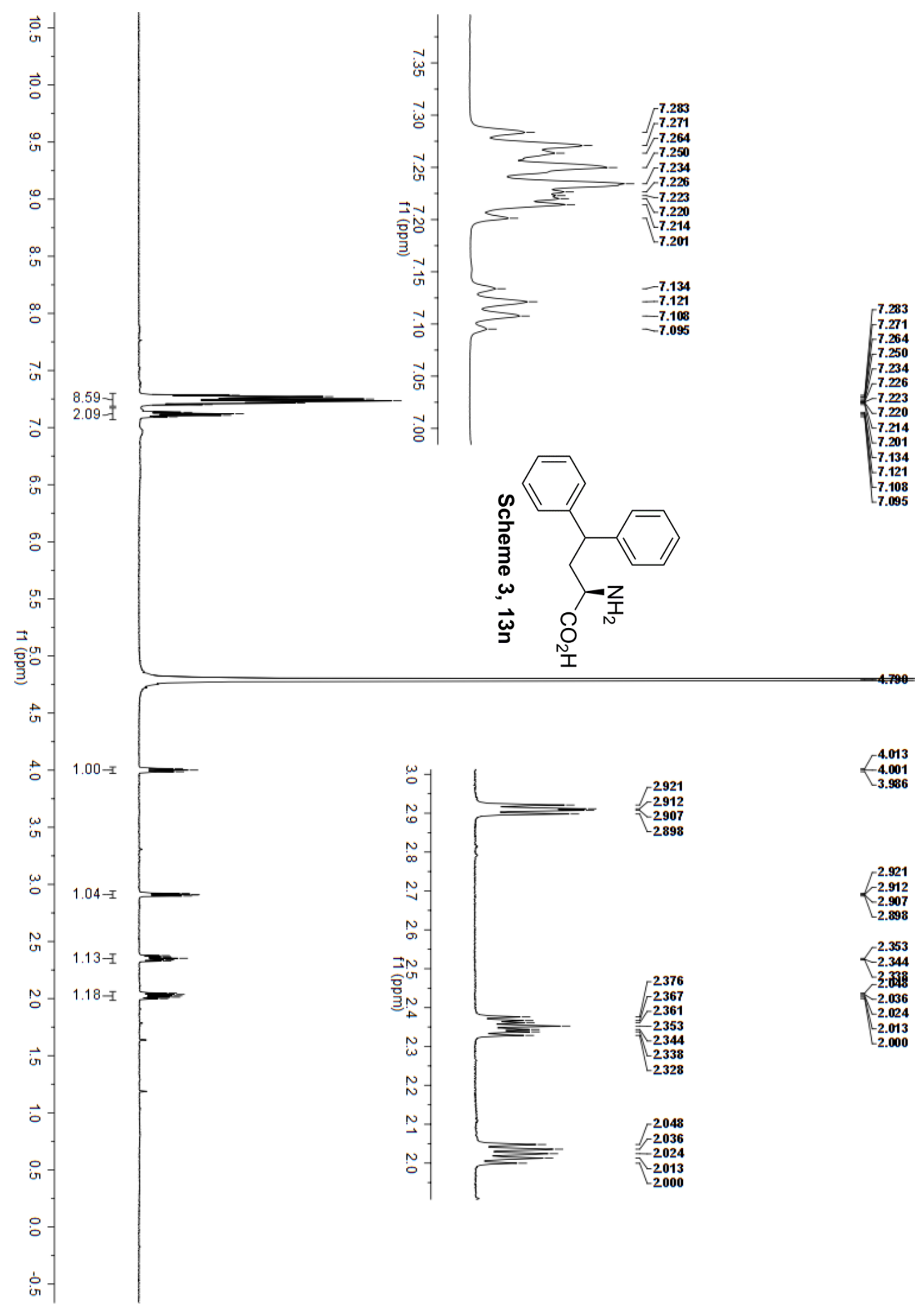




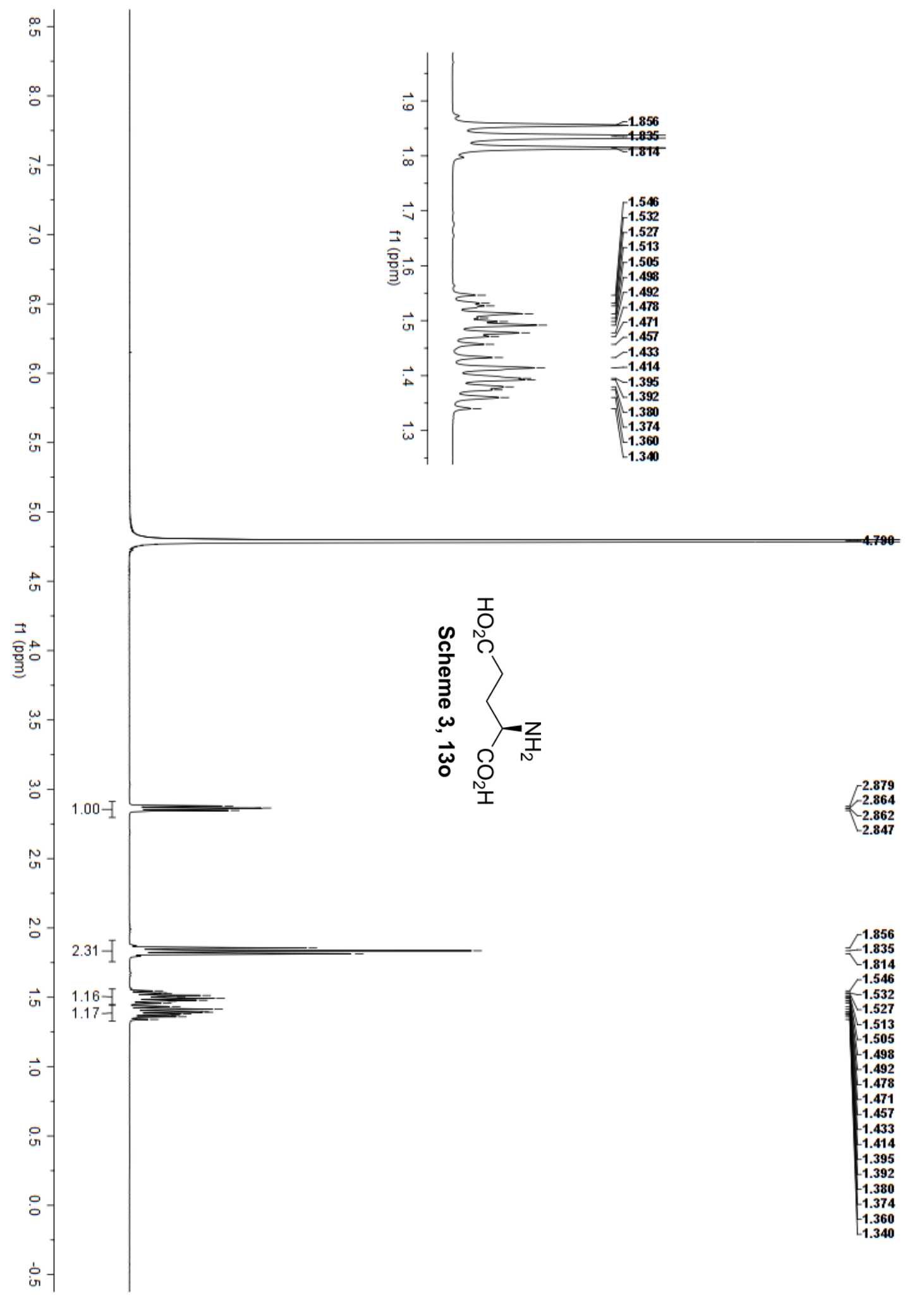




\section{Chromatograms for the Determination of Enantiomeric Excesses}

\section{Scheme 3, compound 13b}<smiles>CCCC(NC(=O)c1ccccc1)C(C)=O</smiles>

\section{HPLC Conditions}

Column: Chiralcel OD-H, Daicel Chemical Industries, Ltd.

Eluent: Hexanes/isopropanol (90:10)

Flow rate: $1.0 \mathrm{~mL} / \mathrm{min}$

Detection: UV $254 \mathrm{~nm}$

\section{Racemic}

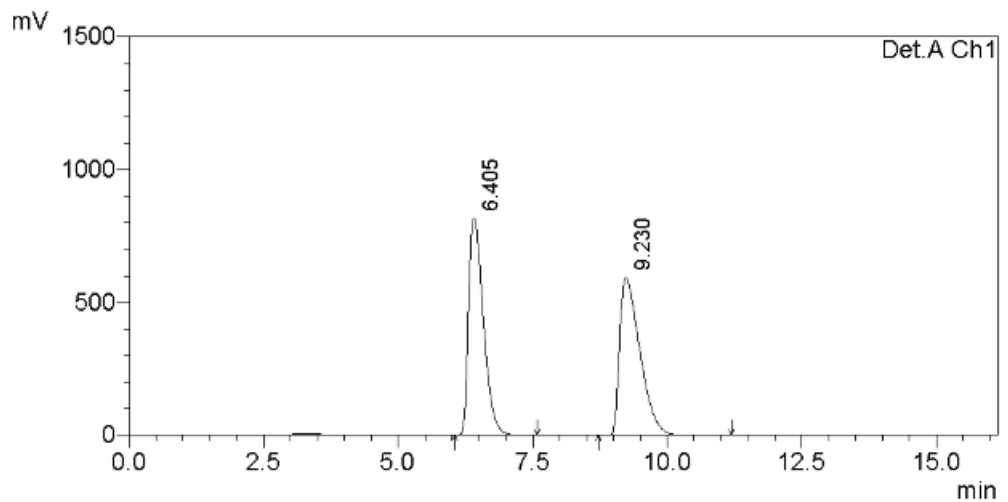

A Ch1 $254 \mathrm{~nm}$
\begin{tabular}{|r|r|r|r|}
\hline Peak\# & Ret. Time & Area & \multicolumn{1}{c|}{ Area $\%$} \\
\hline 1 & 6.405 & 15798565 & 50.013 \\
\hline 2 & 9.230 & 15790102 & 49.987 \\
\hline & & 31588667 & 100.000 \\
\hline
\end{tabular}

\section{Chiral}

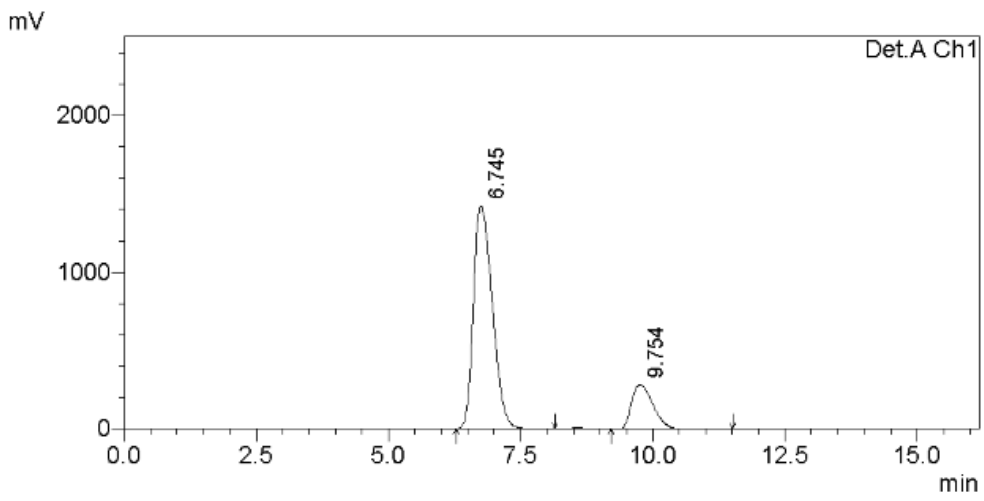

A Ch1 254nm
\begin{tabular}{|r|r|r|r|}
\hline \multicolumn{1}{|c|}{ Peak茾 } & Ret. Time & \multicolumn{1}{|c|}{ Area } & Area $\%$ \\
\hline 1 & 6.745 & 35486852 & 81.334 \\
\hline 2 & 9.754 & 8144410 & 18.666 \\
\hline & & 43631262 & 100.000 \\
\hline
\end{tabular}




\section{Scheme 3, compound 13c}<smiles>CCCC(NC(=O)c1ccccc1)C(C)=O</smiles>

\section{HPLC Conditions}

Column: Chiralcel OD-H, Daicel Chemical Industries, Ltd.

Eluent: Hexanes/isopropanol (94:6)

Flow rate: $1.0 \mathrm{~mL} / \mathrm{min}$

Detection: UV $254 \mathrm{~nm}$

\section{Racemic}

$\mathrm{mV}$

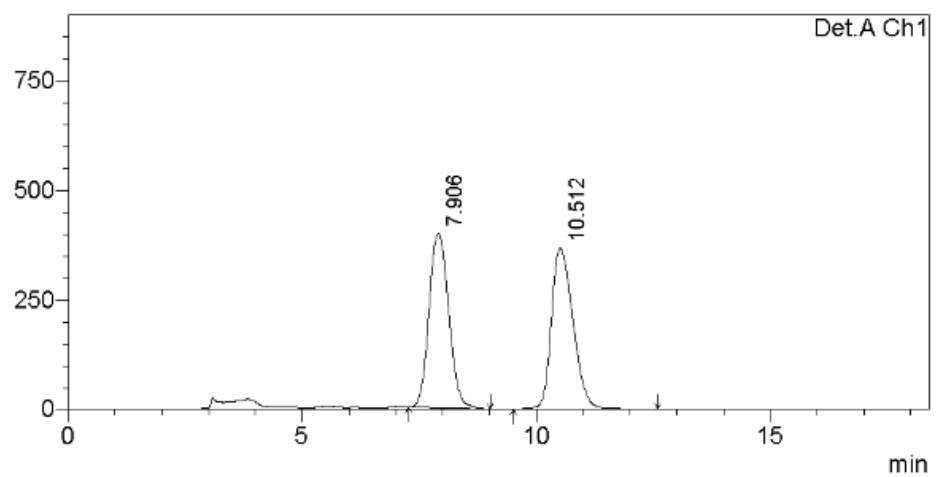

A Ch1 $254 \mathrm{~nm}$
\begin{tabular}{|r|r|r|r|}
\hline \multicolumn{1}{|c|}{ Peak\# } & Ret. Time & Area & \multicolumn{1}{|c|}{ Area $\%$} \\
\hline 1 & 7.906 & 11621077 & 49.653 \\
\hline 2 & 10.512 & 11783522 & 50.347 \\
\hline & & 23404598 & 100.000 \\
\hline
\end{tabular}

\section{Chiral}

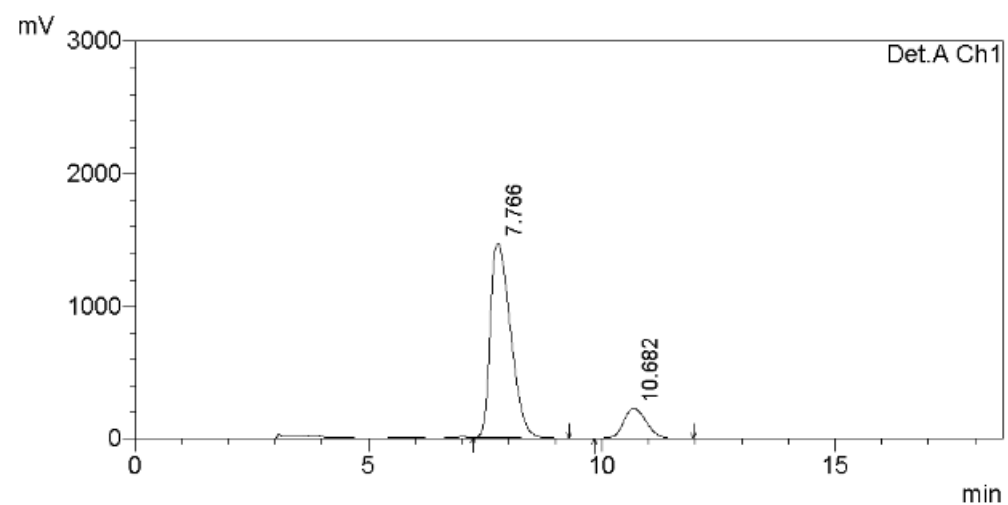

A Ch1 254nm
\begin{tabular}{|r|r|r|r|}
\hline \multicolumn{1}{|c|}{ Peak\# } & Ret. Time & \multicolumn{1}{c|}{ Area } & Area $\%$ \\
\hline 1 & 7.766 & 44487848 & 85.171 \\
\hline 2 & 10.682 & 7745887 & 14.829 \\
\hline & & 52233735 & 100.000 \\
\hline
\end{tabular}




\section{Scheme 3, compound 13d}<smiles>CC=CC(NC(=O)c1ccccc1)C(C)=O</smiles>

\section{HPLC Conditions}

Column: Chiralcel OD-H, Daicel Chemical Industries, Ltd.

Eluent: Hexanes/isopropanol (90:10)

Flow rate: $1.0 \mathrm{~mL} / \mathrm{min}$

Detection: UV $254 \mathrm{~nm}$

\section{Racemic}

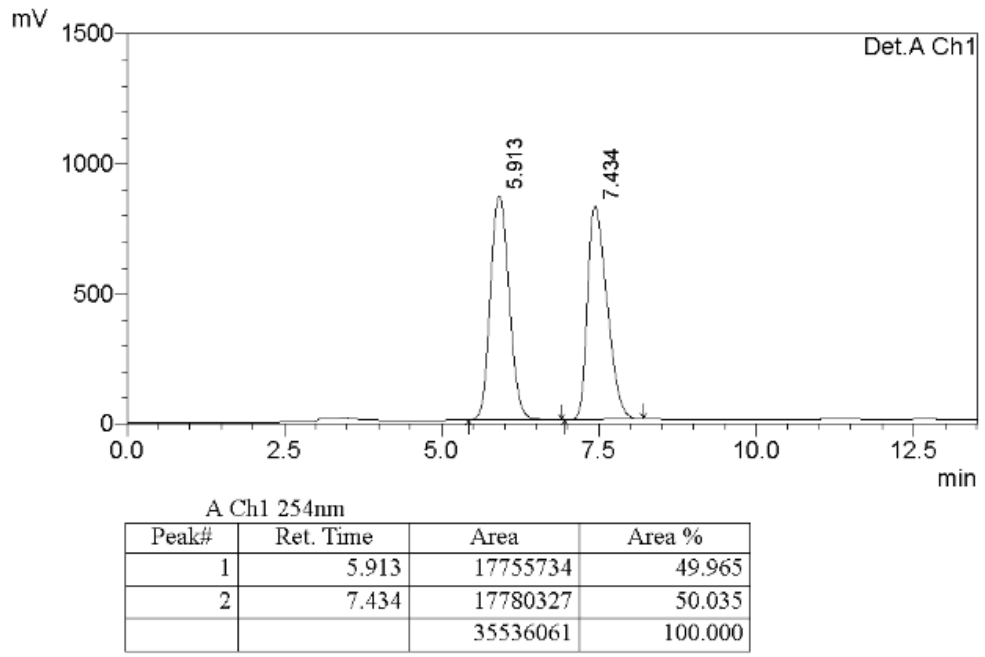

\section{Chiral}

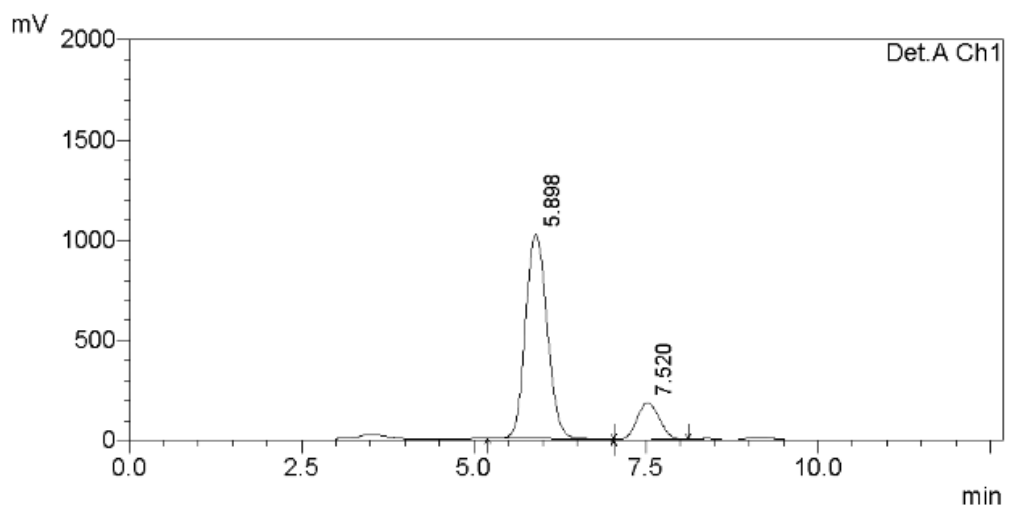

A Chl 254nm
\begin{tabular}{|r|r|r|r|}
\hline \multicolumn{1}{|c|}{ Peak\# } & Ret. Time & \multicolumn{1}{c|}{ Area } & \multicolumn{1}{c|}{ Area \% } \\
\hline 1 & 5.898 & 22520679 & 84.378 \\
\hline 2 & 7.520 & 4169689 & 15.622 \\
\hline & & 26690368 & 100.000 \\
\hline
\end{tabular}




\section{Scheme 3, compound 13e}<smiles>C=CCCC(NC(=O)c1ccccc1)C(C)=O</smiles>

\section{HPLC Conditions}

Column: Chiralcel OD-H, Daicel Chemical Industries, Ltd.

Eluent: Hexanes/isopropanol (97.5:2.5)

Flow rate: $1.0 \mathrm{~mL} / \mathrm{min}$

Detection: UV $254 \mathrm{~nm}$

\section{Racemic}

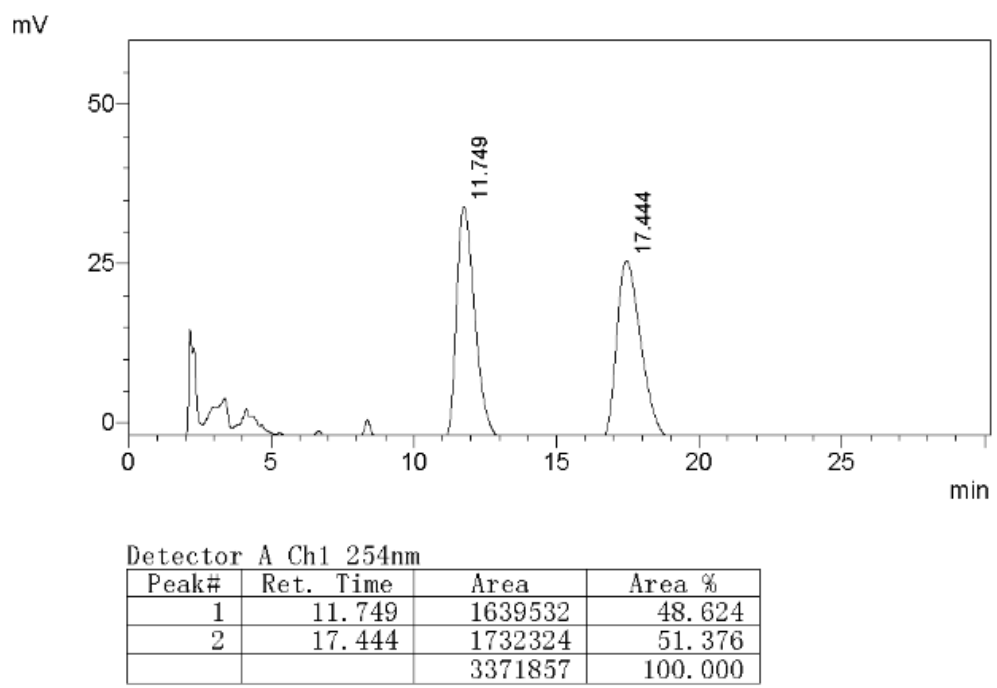

\section{Chiral}

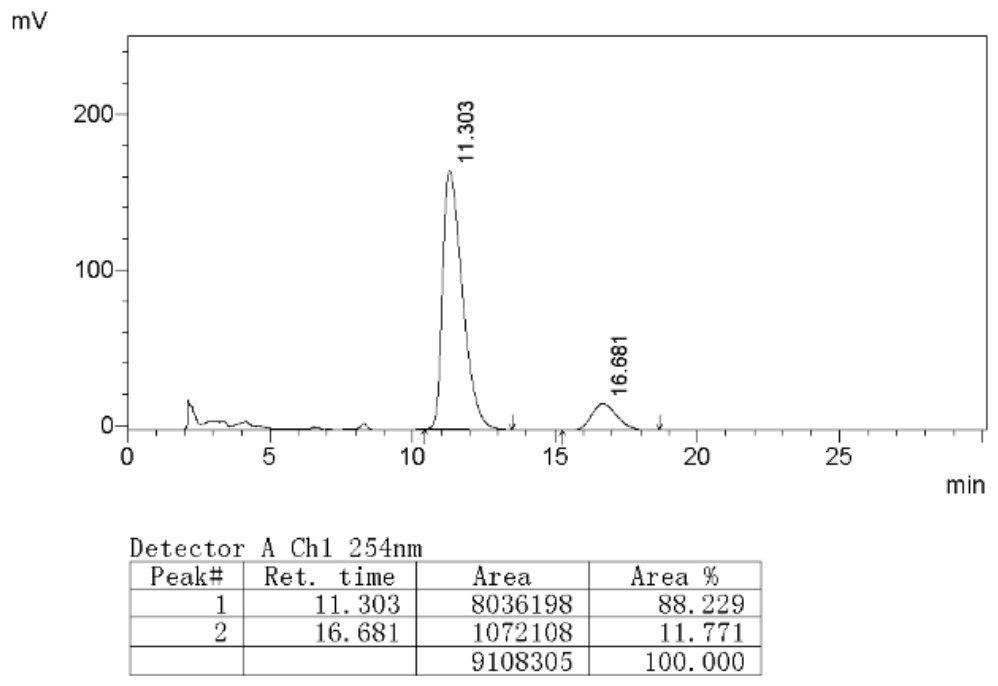


Scheme 3, compound $13 f$<smiles>COC(=O)C(CCOc1ccccc1)NC(=O)c1ccccc1</smiles>

\section{HPLC Conditions}

Column: Chiralcel AD-H, Daicel Chemical Industries, Ltd.

Eluent: Hexanes/isopropanol (85:15)

Flow rate: $1.0 \mathrm{~mL} / \mathrm{min}$

Detection: UV $254 \mathrm{~nm}$

\section{Racemic}

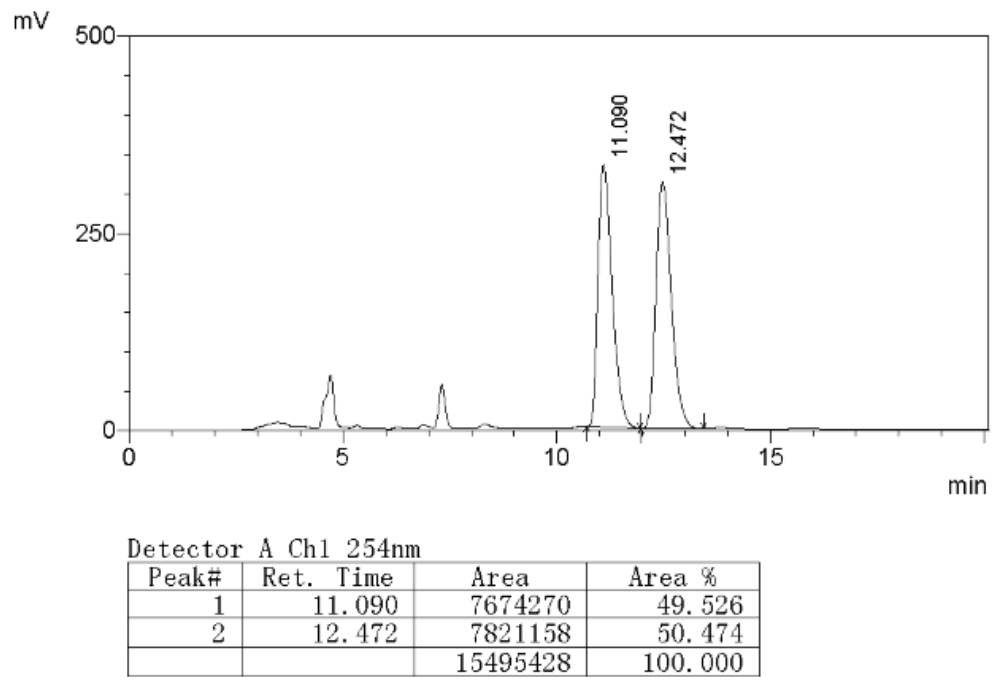

\section{Chiral}

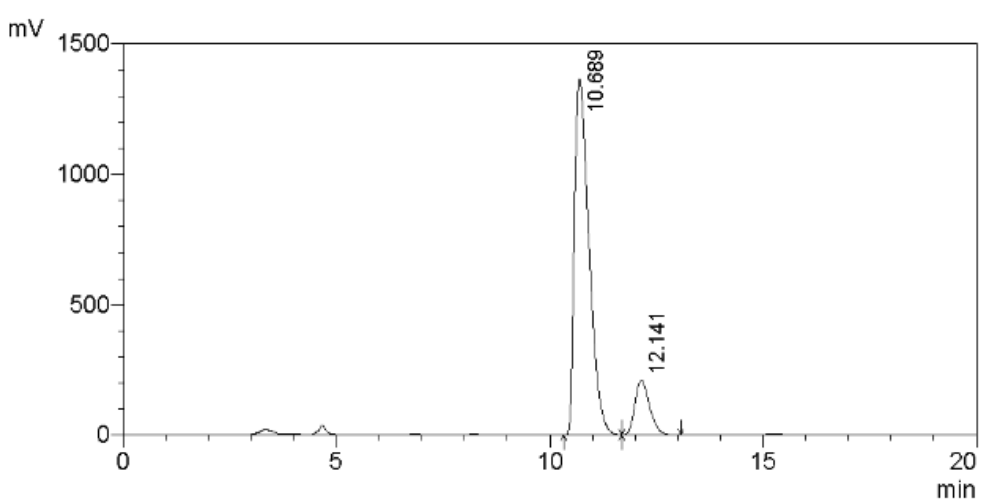

Detecıor A Ch1 $254 \mathrm{~nm}$
\begin{tabular}{|r|r|r|r|}
\hline Peak\# & Ret. Time & \multicolumn{1}{c|}{ Area } & \multicolumn{1}{|c|}{ Area \% } \\
\hline 1 & 10.689 & 32477200 & 86.576 \\
\hline 2 & 12.141 & 5035719 & 13.424 \\
\hline & & 37512919 & 100.000 \\
\hline
\end{tabular}


Scheme 3, compound 13g<smiles>CC(=O)C(CCc1ccccc1-c1ccccc1)NC(=O)c1ccccc1</smiles>

\section{HPLC Conditions}

Column: Chiralcel OD-H, Daicel Chemical Industries, Ltd.

Eluent: Hexanes/isopropanol (90:10)

Flow rate: $1.0 \mathrm{~mL} / \mathrm{min}$

Detection: UV $254 \mathrm{~nm}$

\section{Racemic}

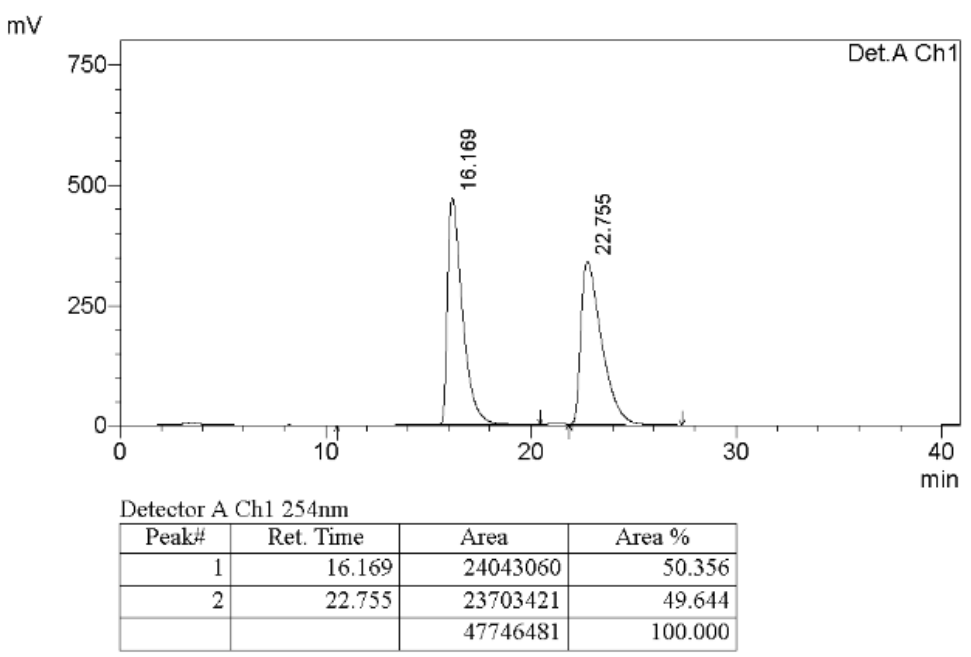

\section{Chairal}

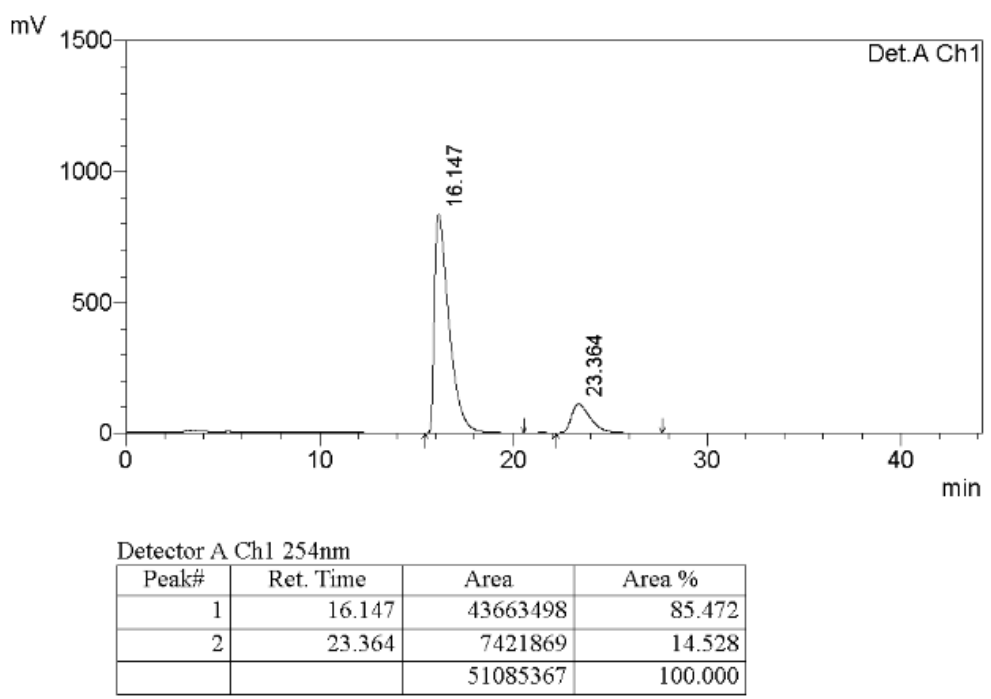


Scheme 3, compound 13h<smiles>CC(=O)C(CCc1c(C)cc(C)cc1C)NC(=O)c1ccccc1</smiles>

\section{HPLC Conditions}

Column: Chiralcel OD-H, Daicel Chemical Industries, Ltd. Eluent: Hexanes/isopropanol (95:5)

Flow rate: $1.5 \mathrm{~mL} / \mathrm{min}$

Detection: UV $254 \mathrm{~nm}$

\section{Racemic}

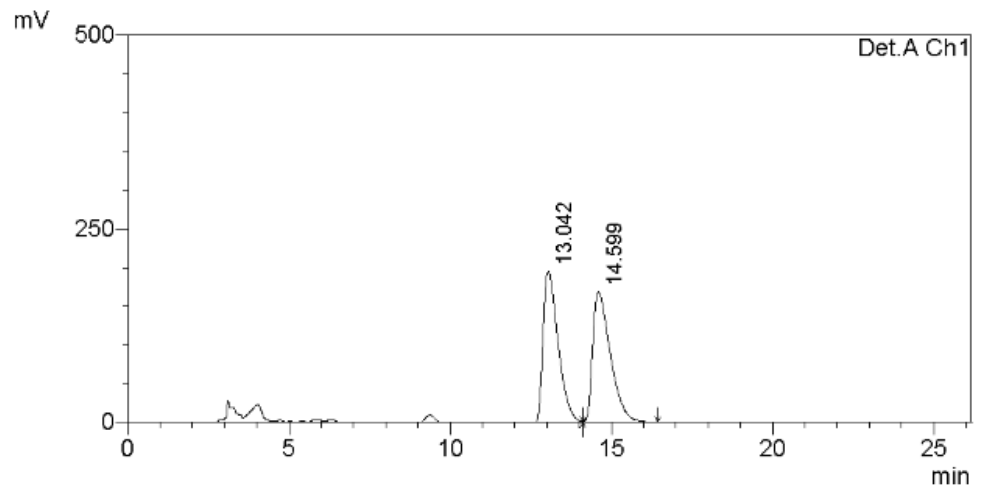

A Ch1 $254 \mathrm{~nm}$
\begin{tabular}{|r|r|r|r|}
\hline Peak\# & Ret. Time & \multicolumn{1}{|c|}{ Area } & Area $\%$ \\
\hline 1 & 13.042 & 6442277 & 49.651 \\
\hline 2 & 14.599 & 6532767 & 50.349 \\
\hline & & 12975044 & 100.000 \\
\hline
\end{tabular}

\section{Chiral}

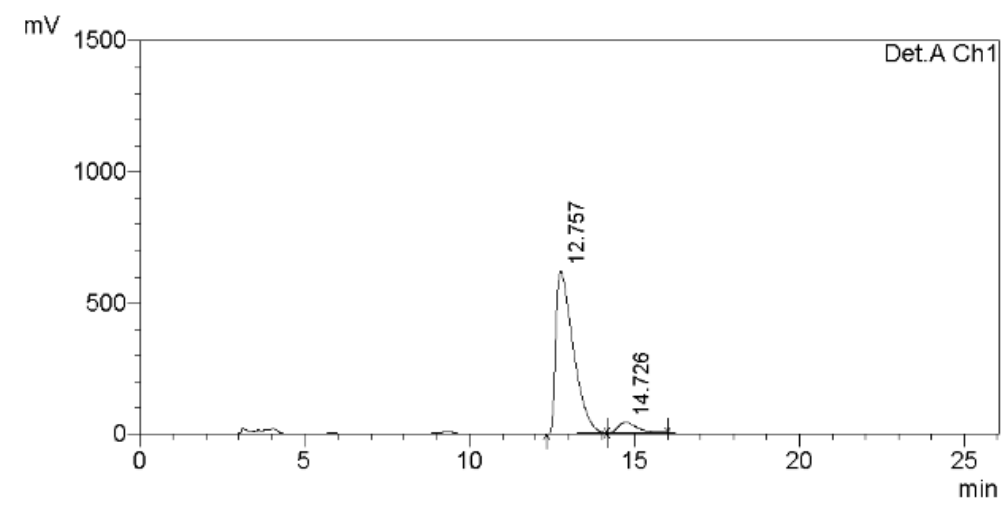

A Ch1 254nm
\begin{tabular}{|r|r|r|r|}
\hline Peak\# & Ret. Time & \multicolumn{1}{|c|}{ Area } & \multicolumn{1}{|c|}{ Area $\%$} \\
\hline 1 & 12.757 & 22907364 & 93.280 \\
\hline 2 & 14.726 & 1650298 & 6.720 \\
\hline & & 24557662 & 100.000 \\
\hline
\end{tabular}


Scheme 3, compound 13a<smiles>CC(=O)[C@H](N)CCc1cccc2ccccc12</smiles>

\section{HPLC Conditions}

Column: Chiralcel OD-H, Daicel Chemical Industries, Ltd.

Eluent: Hexanes/isopropanol (88:12)

Flow rate: $1.5 \mathrm{~mL} / \mathrm{min}$

Detection: UV $254 \mathrm{~nm}$

\section{Racemic}

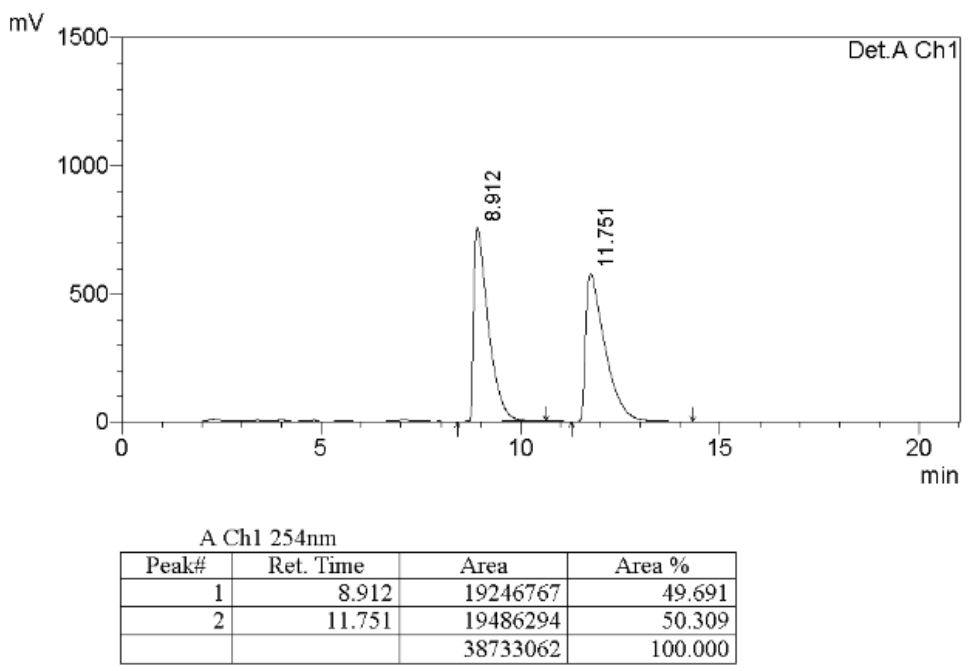

\section{Chiral}

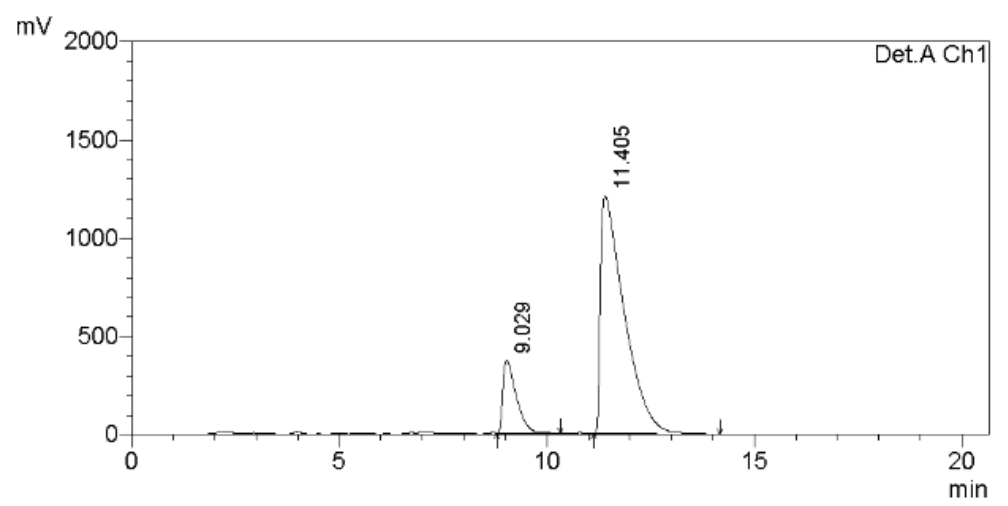

A Ch1 254nm
\begin{tabular}{|r|r|r|r|}
\hline \multicolumn{1}{|c|}{ Peak\# } & Ret. Time & \multicolumn{1}{c|}{ Area } & \multicolumn{1}{c|}{ Area $\%$} \\
\hline 1 & 9.029 & 8345024 & 14.812 \\
\hline 2 & 11.405 & 47995059 & 85.188 \\
\hline & & 56340083 & 100.000 \\
\hline
\end{tabular}


Scheme 3, compound $13 \mathrm{i}$<smiles>CC(=O)C(CCc1ccc2c(c1)CCO2)NC(=O)c1ccccc1</smiles>

\section{HPLC Conditions}

Column: Chiralcel AD-H, Daicel Chemical Industries, Ltd.

Eluent: Hexanes/isopropanol (85:15)

Flow rate: $1.0 \mathrm{~mL} / \mathrm{min}$

Detection: UV $254 \mathrm{~nm}$

\section{Racemic}

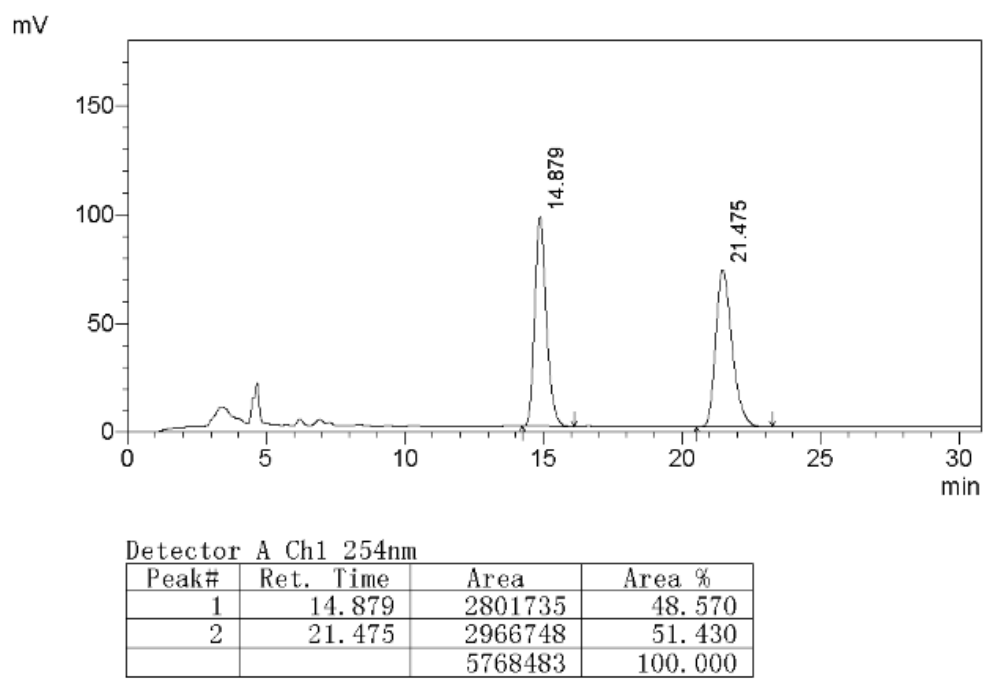

\section{Chiral}

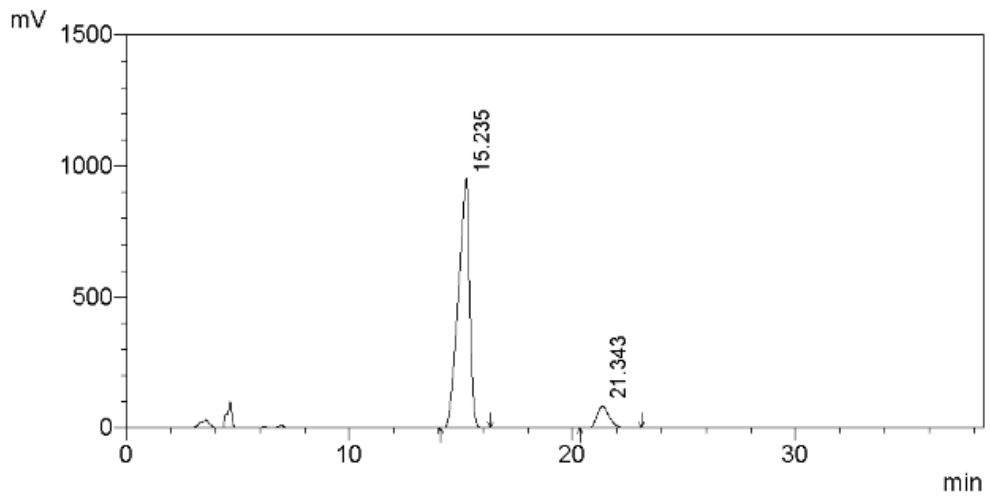

Detector A Ch1 254nm
\begin{tabular}{|r|r|r|r|}
\hline Peak\# & Ret. Time & \multicolumn{1}{c|}{ Area } & \multicolumn{1}{c|}{ Area \% } \\
\hline 1 & 15.235 & 33427465 & 90.521 \\
\hline 2 & 21.343 & 3500242 & 9.479 \\
\hline & & 36927707 & 100.000 \\
\hline
\end{tabular}




\section{Scheme 3, compound 13j}<smiles>CC(=O)[C@H](Cc1ccccc1)NC(=O)c1ccccc1</smiles>

\section{HPLC Conditions}

Column: Chiralcel OD-H, Daicel Chemical Industries, Ltd.

Eluent: Hexanes/isopropanol (95:5)

Flow rate: $1.0 \mathrm{~mL} / \mathrm{min}$

Detection: UV $254 \mathrm{~nm}$

\section{Racemic}

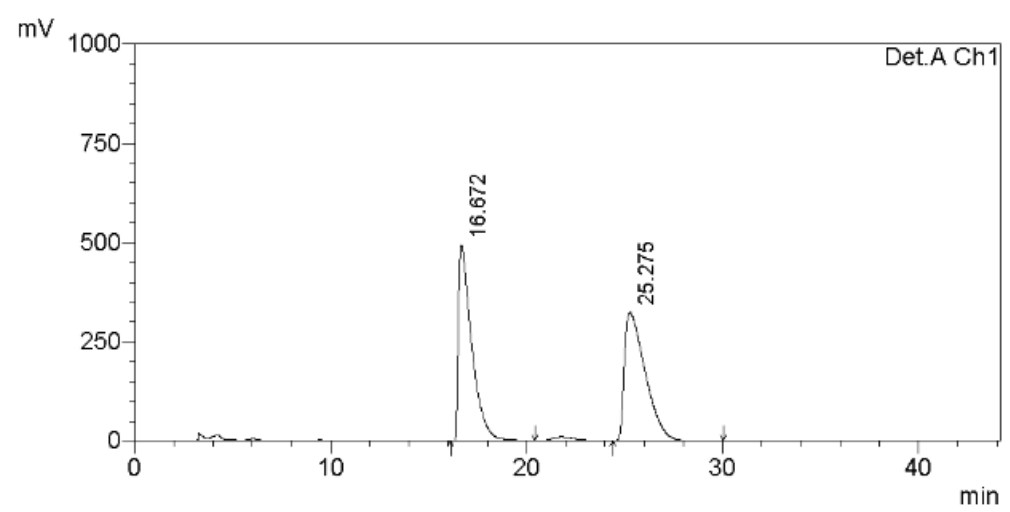

A Ch1 254nm
\begin{tabular}{|r|r|r|r|}
\hline Peak\#̈ & Ret. Time & Area & \multicolumn{1}{|c|}{ Area $\%$} \\
\hline 1 & 16.672 & 23526107 & 49.456 \\
\hline 2 & 25.275 & 24043190 & 50.544 \\
\hline & & 47569297 & 100.000 \\
\hline
\end{tabular}

\section{Chiral}

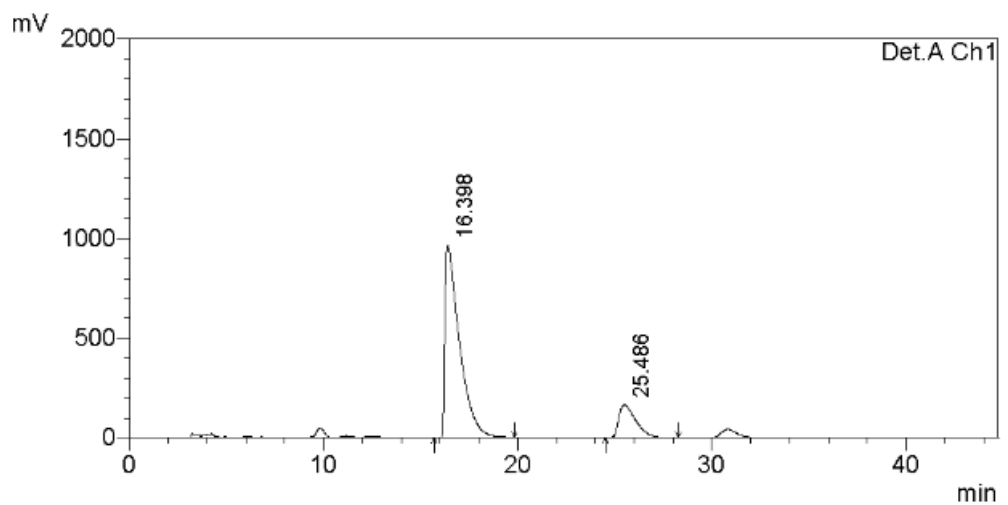

A Ch1 254nm
\begin{tabular}{|r|r|r|r|}
\hline Peak\# & Ret. Time & Area & \multicolumn{1}{|c|}{ Area \% } \\
\hline 1 & 16.398 & 50155174 & 83.187 \\
\hline 2 & 25.486 & 10137157 & 16.813 \\
\hline & & 60292331 & 100.000 \\
\hline
\end{tabular}




\section{Scheme 3, compound 13k}

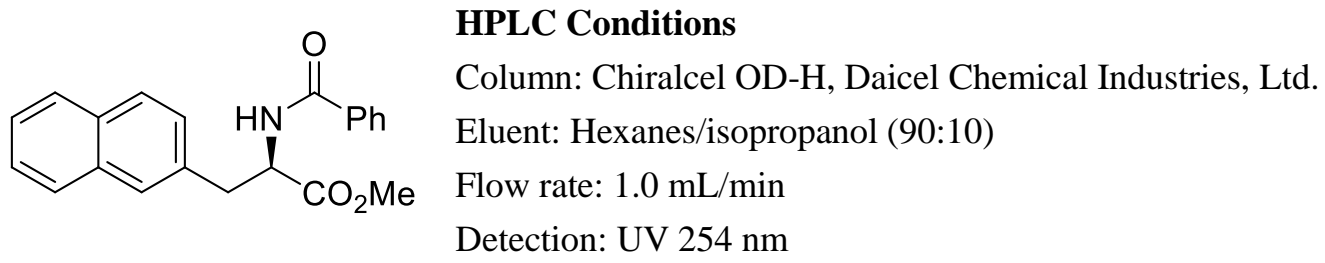

\section{Racemic}

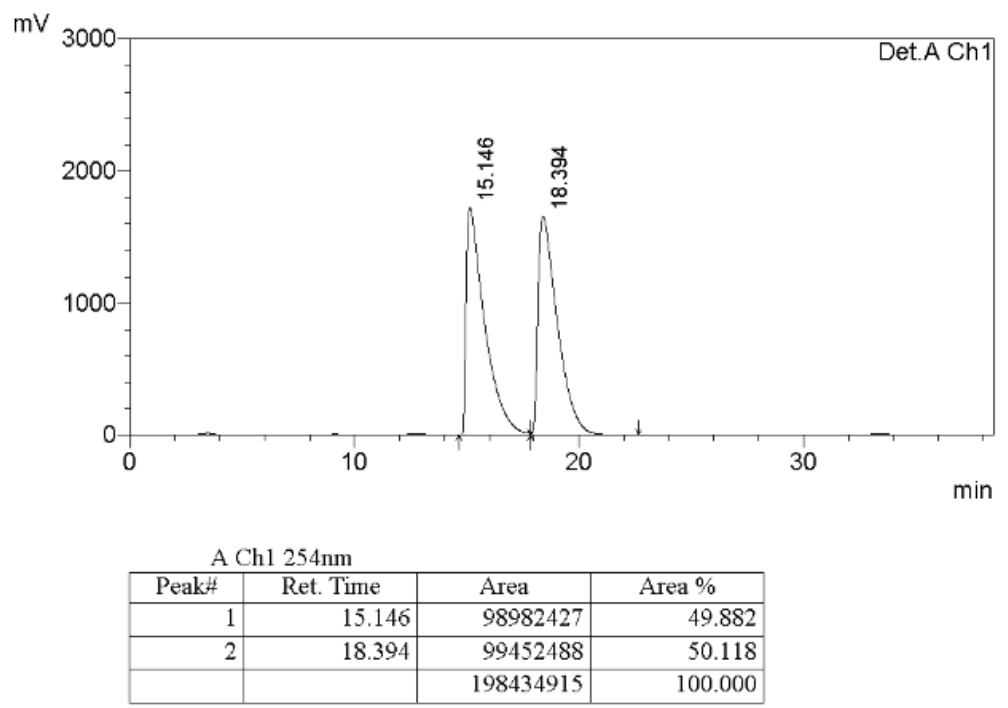

\section{Chiral}

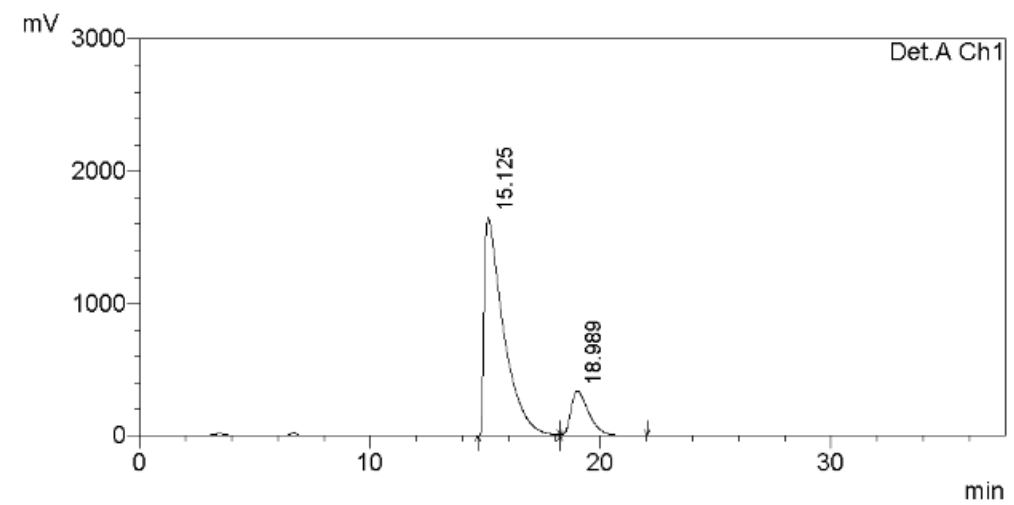

A Ch1 254nm
\begin{tabular}{|r|r|r|r|}
\hline Peak\# & Ret. Time & \multicolumn{1}{c|}{ Area } & Area $\%$ \\
\hline 1 & 15.125 & 96306835 & 84.009 \\
\hline 2 & 18.989 & 18331919 & 15.991 \\
\hline & & 114638753 & 100.000 \\
\hline
\end{tabular}




\section{Scheme 3, compound 131}<smiles>CC(=O)[C@H](CC(C)C)NC(=O)c1ccccc1</smiles>

\section{HPLC Conditions}

Column: Chiralcel OD-H, Daicel Chemical Industries, Ltd.

Eluent: Hexanes/isopropanol (90:10)

Flow rate: $1.0 \mathrm{~mL} / \mathrm{min}$

Detection: UV $254 \mathrm{~nm}$

\section{Racemic}

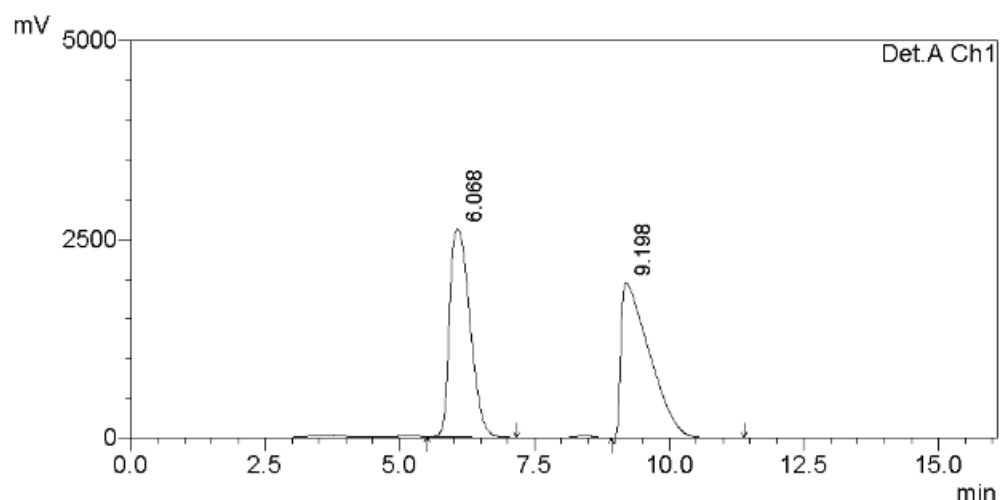

A Ch1 254nm
\begin{tabular}{|r|r|r|r|}
\hline Peak\# & Ret. Time & \multicolumn{1}{|c|}{ Area } & \multicolumn{1}{c|}{ Area \% } \\
\hline 1 & 6.068 & 68641992 & 48.860 \\
\hline 2 & 9.198 & 71844047 & 51.140 \\
\hline & & 140486039 & 100.000 \\
\hline
\end{tabular}

\section{Chiral}

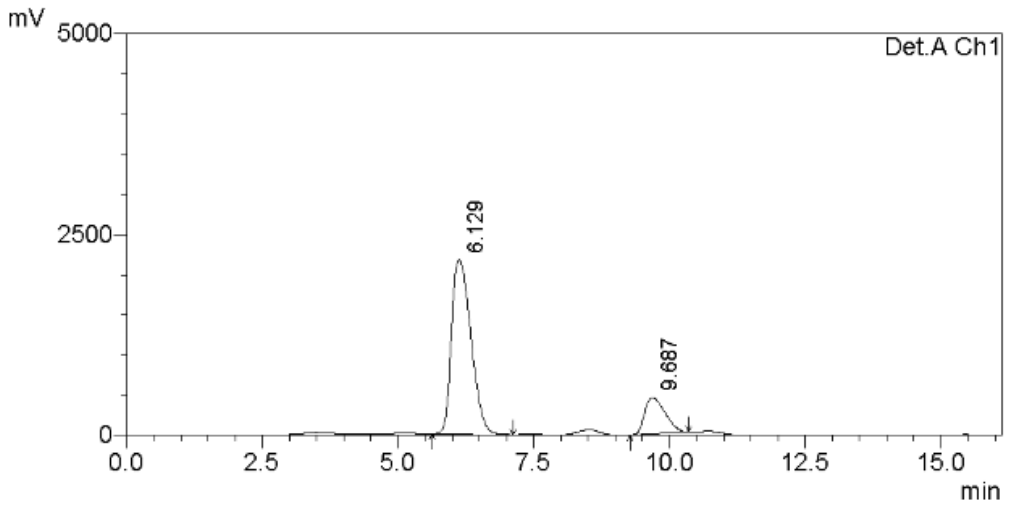

A Ch1 254nm
\begin{tabular}{|r|r|r|r|}
\hline Peak\# & Ret. Time & Area & Area $\%$ \\
\hline 1 & 6.129 & 54223955 & 82.209 \\
\hline 2 & 9.687 & 11734934 & 17.791 \\
\hline & & 65958889 & 100.000 \\
\hline
\end{tabular}




\section{Scheme 3, compound 13m}<smiles>CCC(CC)C[C@H](NC(=O)c1ccccc1)C(C)=O</smiles>

\section{HPLC Conditions}

Column: Chiralcel OD-H, Daicel Chemical Industries, Ltd.

Eluent: Hexanes/isopropanol (95:5)

Flow rate: $1.0 \mathrm{~mL} / \mathrm{min}$

Detection: UV $254 \mathrm{~nm}$

\section{Racemic}

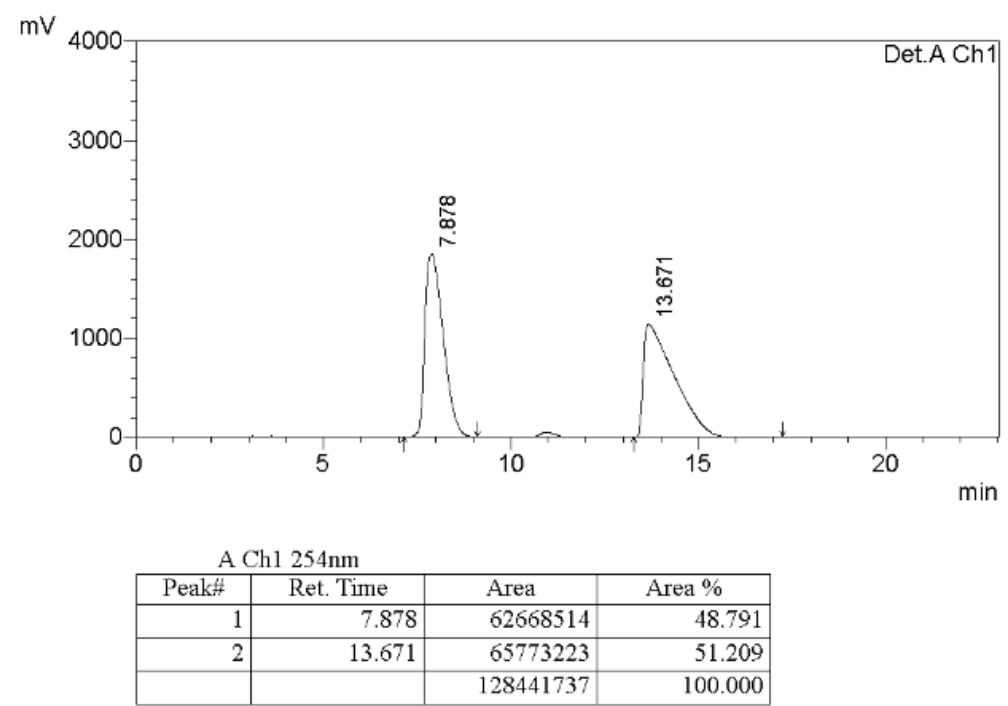

\section{Chiral}

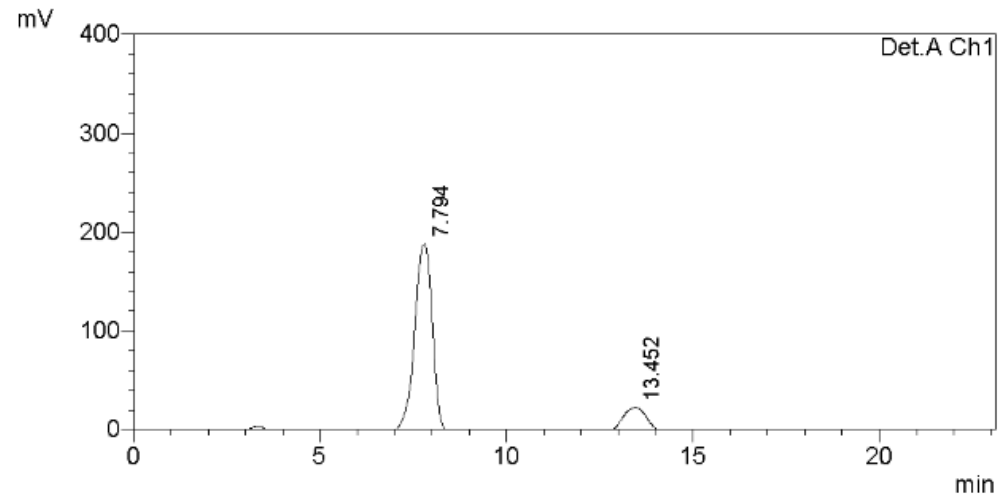

A Ch1 254nm
\begin{tabular}{|r|r|r|r|}
\hline Peak\# & Ret. Time & \multicolumn{1}{c|}{ Area } & \multicolumn{1}{c|}{ Area \% } \\
\hline 1 & 7.794 & 6402016 & 84.853 \\
\hline 2 & 13.452 & 1142815 & 15.147 \\
\hline & & 7544831 & 100.000 \\
\hline
\end{tabular}




\section{Scheme 3, compound 13n}<smiles>CC(=O)[C@H](CC(c1ccccc1)c1ccccc1)NC(=O)c1ccccc1</smiles>

\section{HPLC Conditions}

Column: Chiralcel AD-H, Daicel Chemical Industries, Ltd.

Eluent: Hexanes/isopropanol (85:15)

Flow rate: $1.0 \mathrm{~mL} / \mathrm{min}$

Detection: UV $254 \mathrm{~nm}$

\section{Racemic}

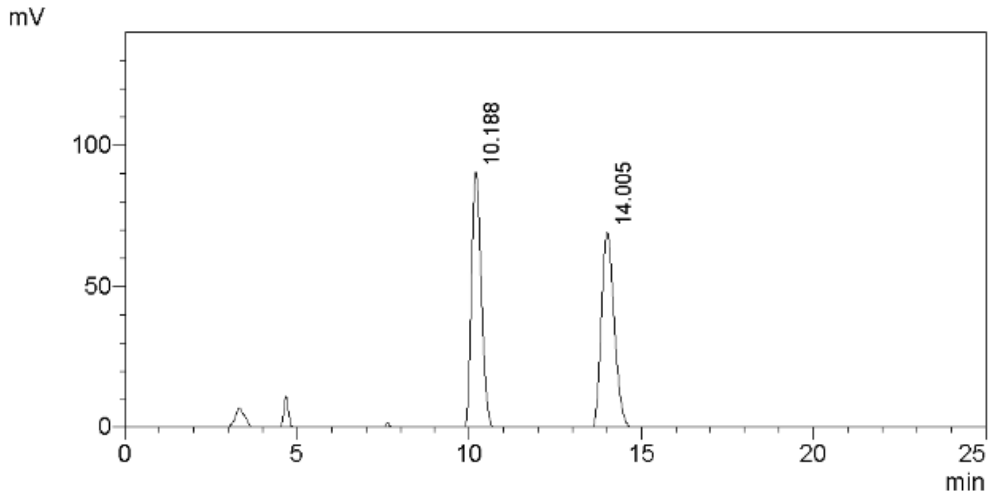

Detector A Ch1 254nm
\begin{tabular}{|r|r|l|r|}
\hline Peak\# & Ret. Time & Area & Area \% \\
\hline 1 & 10.188 & 1924808 & 50.311 \\
\hline 2 & 14.005 & 1901032 & 49.689 \\
\hline & & 3825840 & 100.000 \\
\hline
\end{tabular}

\section{Chiral}

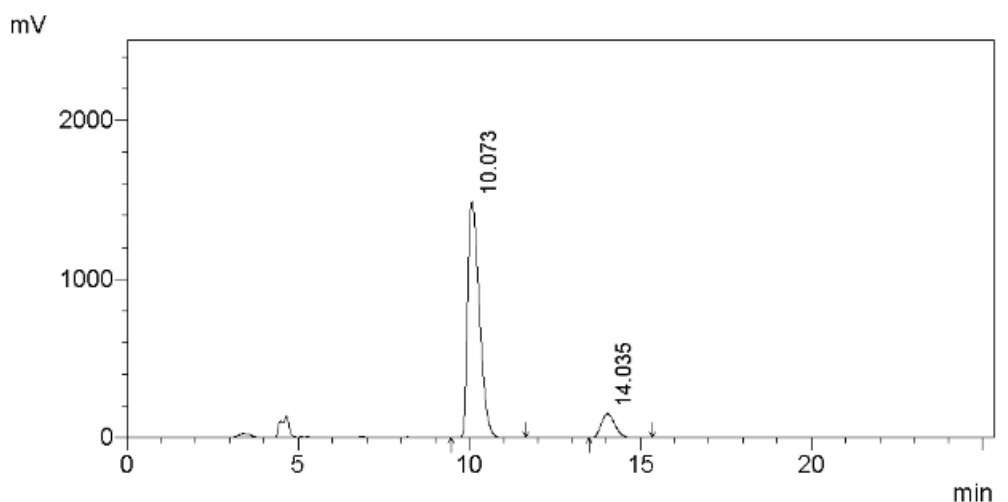

Detector A Ch1 $254 \mathrm{~nm}$
\begin{tabular}{|r|r|r|r|}
\hline Peak \# & Ret. Time & \multicolumn{1}{c|}{ Area } & Area \% \\
\hline 1 & 10.073 & 35494769 & 89.065 \\
\hline 2 & 14.035 & 4358103 & 10.935 \\
\hline & & 39852872 & 100.000 \\
\hline
\end{tabular}




\section{Scheme 3, compound 130}

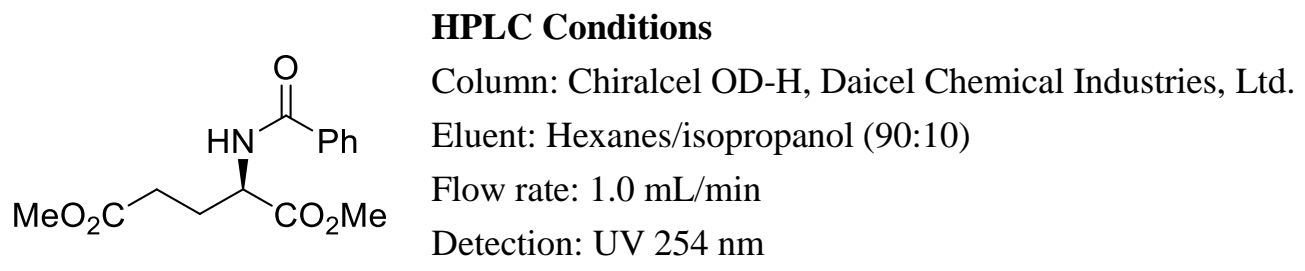

\section{Racemic}

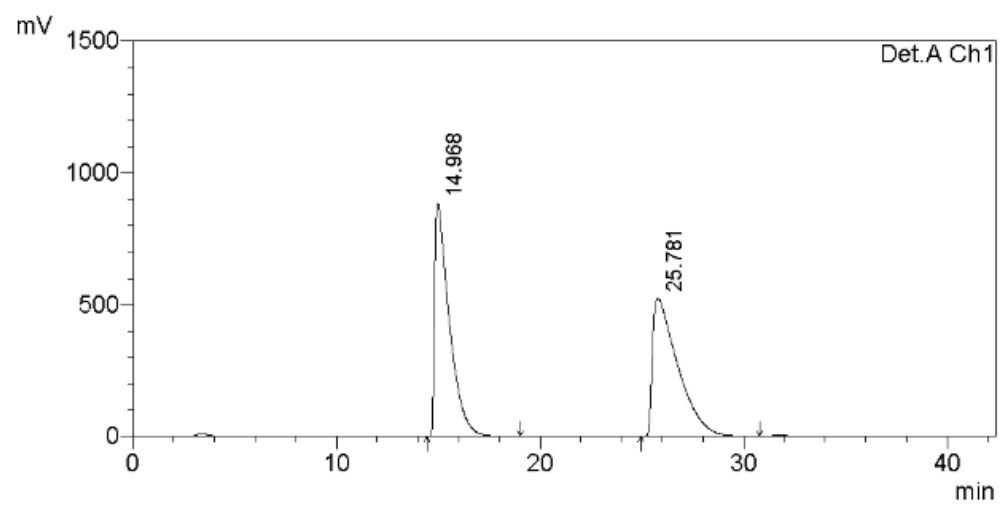

A Ch1 254nm
\begin{tabular}{|r|r|r|r|}
\hline Peak\# & Ret. Time & Area & \multicolumn{1}{c|}{ Area $\%$} \\
\hline 1 & 14.968 & 44633153 & 49.577 \\
\hline 2 & 25.781 & 45394126 & 50.423 \\
\hline & & 90027279 & 100.000 \\
\hline
\end{tabular}

\section{Chiral}

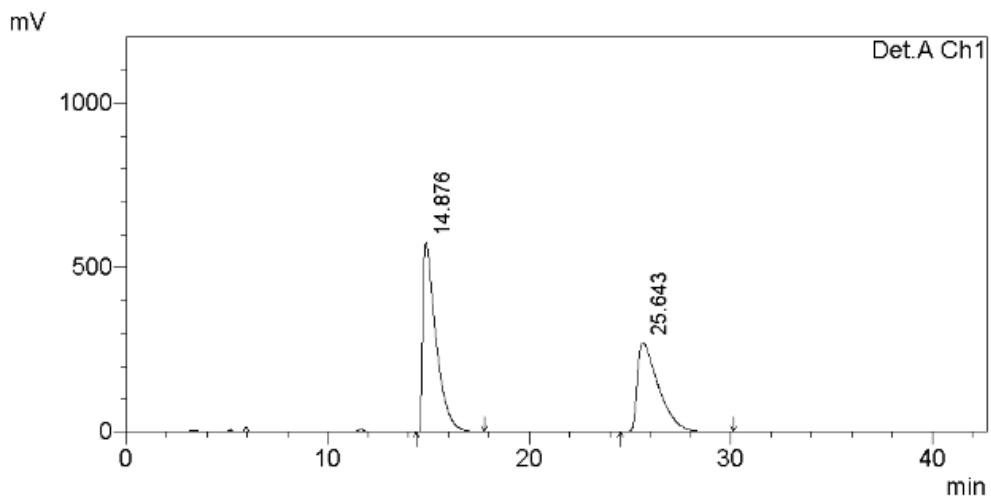

A Ch1 254nm
\begin{tabular}{|r|r|l|r|}
\hline \multicolumn{1}{|c|}{ Peak\# } & Ret. Time & \multicolumn{1}{c|}{ Area } & \multicolumn{1}{c|}{ Area \% } \\
\hline 1 & 14.876 & 26251523 & 57.102 \\
\hline 2 & 25.643 & 19721472 & 42.898 \\
\hline & & 45972994 & 100.000 \\
\hline
\end{tabular}

